Florida International University FIU Digital Commons

$10-13-2003$

\title{
Design and optimization of long term monitoring systems for contaminated environmental sites
}

Amit Deshpande

Florida International University

DOI: $10.25148 /$ etd.FI14062250

Follow this and additional works at: https://digitalcommons.fiu.edu/etd

Part of the Computer Engineering Commons

\section{Recommended Citation}

Deshpande, Amit, "Design and optimization of long term monitoring systems for contaminated environmental sites" (2003). FIU Electronic Theses and Dissertations. 2780.

https://digitalcommons.fiu.edu/etd/2780 
FLORIDA INTERNATIONAL UNIVERSITY

Miami, Florida

DESIGN AND OPTIMIZATION OF

LONG TERM MONITORING SYSTEMS FOR CONTAMINATED

ENVIRONMENTAL SITES

A thesis submitted in partial fulfillment of the

requirements for the degree of

MASTER OF SCIENCE

in

COMPUTER ENGINEERING

by

Amit Deshpande

2003 
To: Dean Vish Prasad

College of Engineering

This thesis, written by Amit Deshpande, and entitled Design and Optimization of . Long Term Monitoring Systems for Contaminated Environmental Sites, having been approved in respect to style and intellectual content, is referred to you for judgment.

We have read this thesis and recommend that it be approved.

M. A. Ebadian

David Roelant

Malcolm Heimer

Marshall Allen

Subbarao Wunnava, Major Professor

Date of Defense: October 13, 2003

The thesis of Amit Deshpande is approved.

Dean Douglas Wartzok University Graduate School

Florida International University, 2003 


\section{DEDICATION}

I dedicate this research work to my beloved parents, dear brother, friends and committee members who supported and helped me throughout this period. 


\section{ACKNOWLEDGMENTS}

I express a sincere gratitude to my committee members, Dr. Subbarao Wunnava, Dr. M. A. Ebadian, Mr. Marshall Allen, Dr. David Roelant and Dr. Malcolm Heimer for their invaluable comments, assistance and interest in the completion of this thesis. I am particularly grateful to Dr. David Roelant and Mr. Marshall Allen at the Hemispheric Center for Environmental Technology (HCET) for giving me an opportunity to work with them and funding this research project. 


\author{
ABSTRACT OF THE THESIS \\ DESIGN AND OPTIMIZATION OF \\ LONG TERM MONITORING SYSTEMS FOR CONTAMINATED \\ ENVIRONMENTAL SITES \\ by \\ Amit Deshpande \\ Florida International University, 2003 \\ Miami, Florida \\ Professor Subbarao Wunnava, Major Professor
}

The purpose of this study was to design and optimize long-term monitoring systems for contaminated environmental sites. This study identified some major issues related to long-term monitoring systems, including those related to sensors, communications, security, and data acceptability. The state of the art among the various components was detailed with benefits and limitations identified for all. It focused on how optimization of performance criteria is achieved in automated long-term monitoring systems. This study particularly focused on a trade-off analysis for determining the optimal long-term monitoring system design. A set of long-term monitoring scenarios was presented and the optimized long-term monitoring system design was then suggested.

Reviews of commercially available water quality monitoring sensors for volatile organic compounds and Supervisory Control and Data Acquisition (SCADA) systems were included. 
By using automated systems as suggested in this thesis significant cost reduction may be achieved over the baseline sampling approach. It concludes with several optimized scenarios that can be used for assessing the success of monitored natural attenuation of contaminated groundwater monitoring. 


\section{TABLE OF CONTENTS}

CHAPTER

1. INTRODUCTION

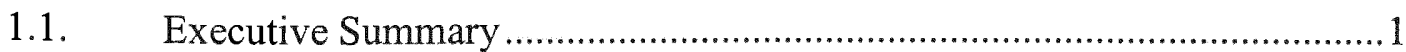

1.2. Background - Contaminated Sites and Need for LTM .............................5

1.2.1. Differences - Process Optimization Monitoring and LTM...................... 7

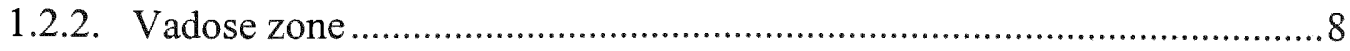

1.2.3. Characterizing Contamination ............................................................. 9

1.2.4. Reducing Long-Term Monitoring Costs.............................................. 9

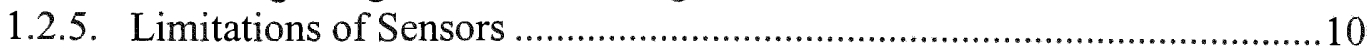

1.2.6. Placing, Replacing, and Calibrating Sensors .........................................10

1.3. Long-Term Monitoring System Design ..................................................... 11

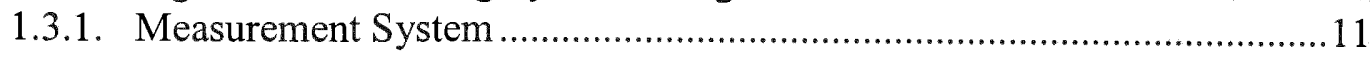

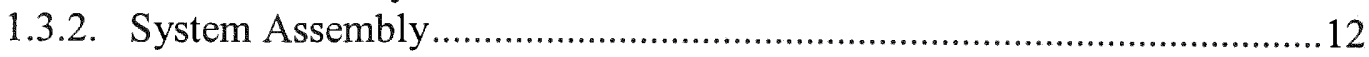

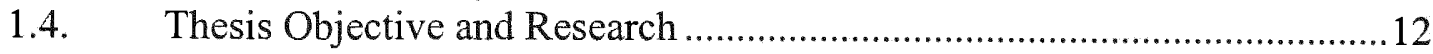

1.5. Theoretical Perspective - Component Design of LTM ..............................13

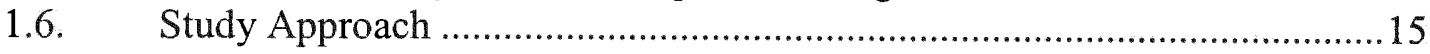

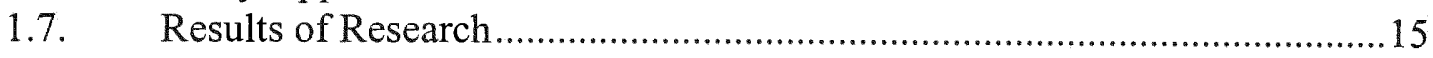

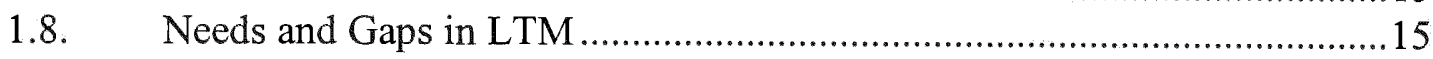

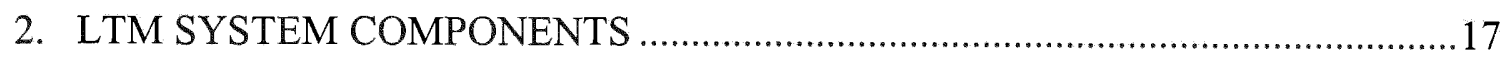
SECTION I

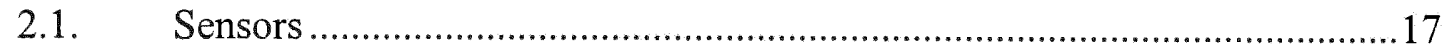

2.2. Contaminant Measurement Techniques..................................................18

2.2.1. Typical Contaminants of Concern .........................................................18

2.2.2. Sensors used for Volatile Organic Compounds .......................................18

2.2.3. Moisture Data...................................................................................2

2.3. Issues about Sensors and Field Analytical Methods ...............................29

2.4. Issues related particularly to Sensors .......................................................29

2.5. Indicator Parameters: Example of LTM for Monitored Natural

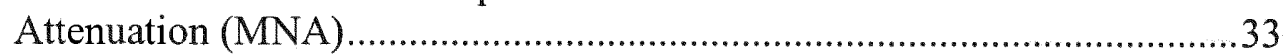

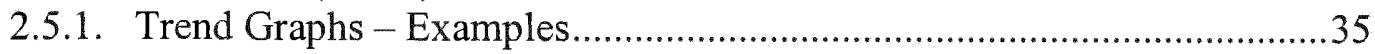

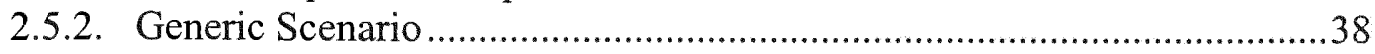

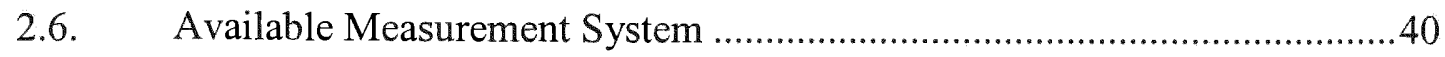

SECTION II

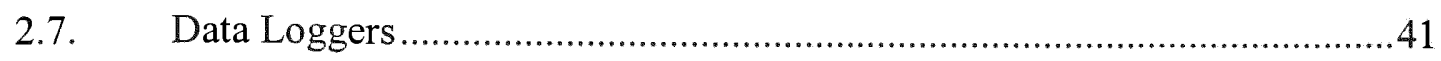

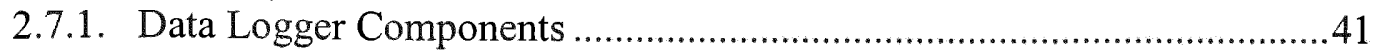

2.7.2. Operation of a Data Logger ...................................................................4 41

2.7.3. Data Loggers as part of Environmental Monitoring System ...................42

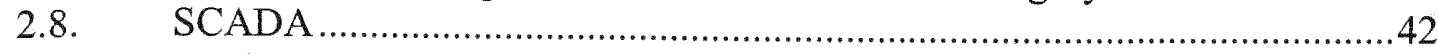

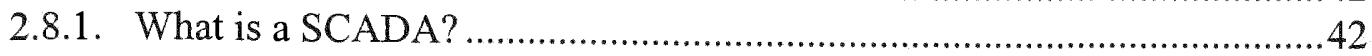

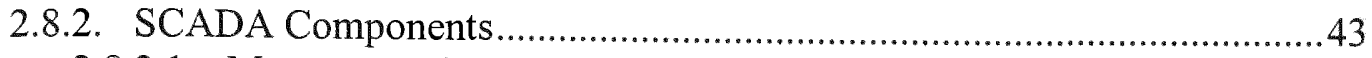

2.8.2.1. Master Terminal Unit.....................................................................4 
2.8.2.2. Remote terminal Unit..................................................................4

2.8.3. Communication between RTU and MTU ….........................................44

2.8.4. Important Applications of SCADA …….................................................49

2.8.5. Differences between Distributed Control Systems (DCS) and

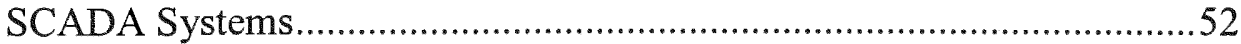

2.8.6. Selection of the Appropriate SCADA Package .......................................53

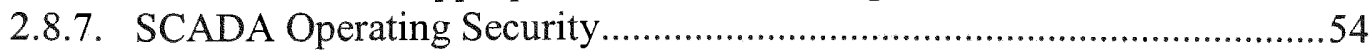

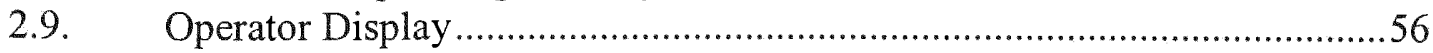

3. OPTIMIZATION OF LTM SYSTEMS FOR MONITORED NATURAL NATURAL ATTENUATION (MNA) ….................................................58

3.1. Monitored Natural Attenuation............................................................58

3.2. Issues Related to Traditional Technology.................................................59

3.3. Comparative Analysis of Long-Term Monitoring Strategies .....................60

3.3.1. Diagram of Contaminated Groundwater Plume with Monitoring Wells for Monitoring MNA Process Activity..........................................60

3.3.2. LTM Performance Criteria .....................................................................61

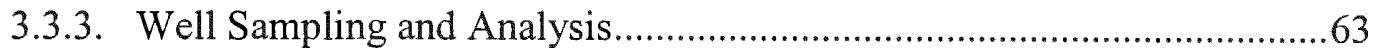

3.3.4. Well Sampling and Multi-probe Sensor ...................................................64

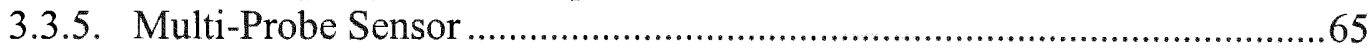

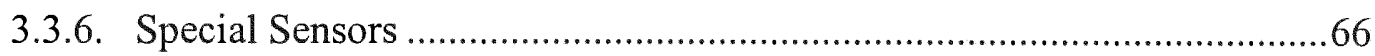

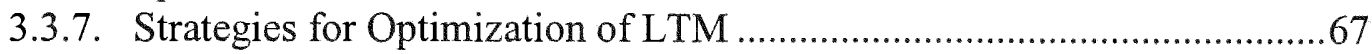

3.3.7.1. LTM System with Network of Multi-Probe Sensors ........................68

3.3.7.2. Actual Implementation of the Strategy .............................................73

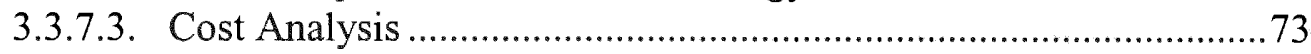

3.3.7.4. LTM System with Network of Special Sensors................................75

3.3.8. Optimization with respect to LTM Performance Criteria for Specific

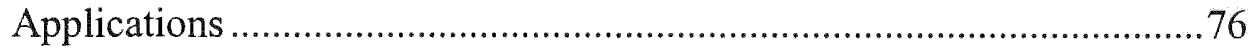

3.3.8.1. Optimization using LTM System with Multi-Probe Sensor for a

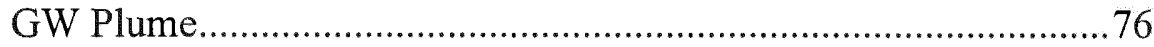

3.3.8.2. Optimization using LTM System with Special Sensors for a

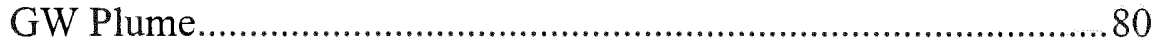

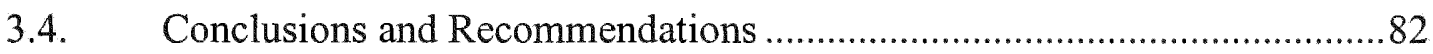

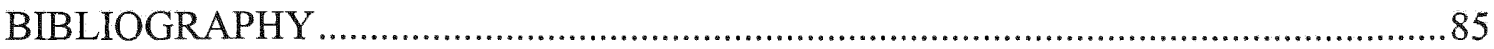

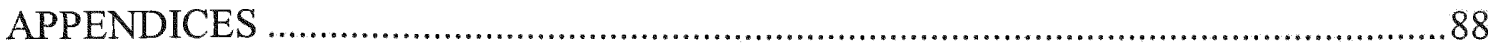




\section{LIST OF FIGURES}

FIGURE

PAGE

Figure 1. General Schematic of Long-Term Monitoring System 6

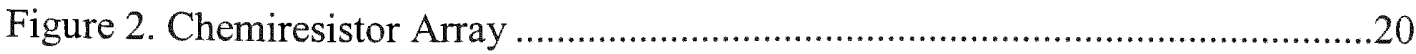

Figure 3. Catalytic Bead Sensor...............................................................22

Figure 4. Schematic of Surface Acoustic Device ............................................23

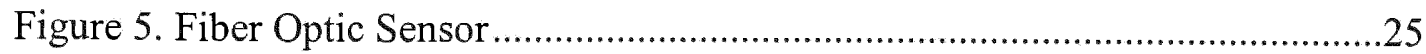

Figure 6. Common Patterns of Chlorinated Solvent Biodegradation in an Anaerobic System ................................................................... 35

Figure 7. Common Patterns of Chlorinated Solvent Biodegradation in a Sequential Aerobic/Anaerobic System ............................................................ 36

Figure 8. General Scenario of Contaminated Groundwater System.......................38

Figure 9. Typical Long Term Monitoring System ............................................39

Figure 10. Master Terminal Unit - Interfaces to RTU and other devices ...............44

Figure 11. Remote Terminal Unit - Interfaces to MTU and other devices .............45

Figure 12. Point-to-Point Configuration ...................................................45

Figure 13. Point-to-Multipoint Configuration ................................................46

Figure 14. Omni-Directional Antennas.....................................................48

Figure 15. Yagi Antennas ...................................................................4 48

Figure 16. The Contaminated Groundwater Plume .............................................60

Figure 17. Possible RTU Arrangement for Typical Example................................70

Figure 18. Graph of total cost per fifteen hundred wells versus the number of years..

Figure 19. Groundwater Plume with Multi-Probe Sensors and SCADA System......77 
Figure 20. miniTroll sensors in the field ..............................................................

Figure 21. Individual installation of a Troll 9000 or miniTroll sensor .....................79

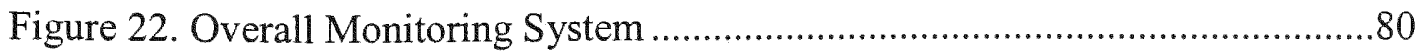




\section{CHAPTER 1 - INTRODUCTION}

\subsection{Executive Summary}

During the last decade, over 1200 hazardous waste sites in the US have been placed on the EPA National Priority List for remedial investigation and cleanup. There are numerous contaminants that are cause of concern at these sites. The focus of this thesis is long-term monitoring of hazardous Volatile Organic Compounds (VOCs) in soils and groundwater that pose a major threat to groundwater supplies.

After site cleanup has ended some form of post-closure monitoring will continue due to regulatory agency requirements, typically for 30 years or more. Long-term monitoring (LTM) associated with these sites can be an expensive endeavor. The reason for the high cost is that the traditional approach involves sample collection, storage, lab analysis, data validation and reporting. These components of monitoring have major labor costs.

The main purpose of this research is to design and optimize long-term automated monitoring systems for contaminated environmental sites; these systems collect data using measurement technologies, transmit the data to a central location and display the data to the operator. This "real-time" data provides an instant picture of the residual contaminants left at these cleaned sites. These systems also provide storage for the information that can be retrieved later for trend analysis or comparisons with the current data.

This research has wide applicability to many sites across the country and in particular to sites at the Department of Energy's Savannah River Site (SRS). This HCET 
project for SRS is providing research and design for a long-term monitoring system for contaminated groundwater in a monitored natural attenuation application. The main contaminants in the groundwater are perchloroethylene (PCE) and trichloroethylene (TCE).

This research analyses the various components of a long-term monitoring system in order to develop an optimal design. The major components of a LTM system are:

(1) Measurement System - Sensors

(2) Data Acquisition System

(3) Communication System

The sensors that are used for measuring VOCs like trichloroethylene, perchloroethylene (PCE), and breakdown products of these contaminants. Issues associated with these sensors are also analyzed. One of the alternatives for the selection of sensors is to choose sensors that monitor indicator or surrogate parameters such as $\mathrm{pH}$, oxidation-reduction potential, chloride, nitrate, and sulfate. The indicator parameter measurements could be better indicators of early system failures than direct contaminant measurements. How one can infer from indicator parameters concentrations whether the actual contaminant is degrading over time is presented.

Different types of commercially available data acquisition systems (DAS) and their components have been studied in detail. Data Loggers are one type of DAS. Supervisory Control \& Data Acquisition Systems i.e., SCADA, are another type of data acquisition system normally used for monitoring processes that occupy a large geographical area. SCADA systems allow an operator at a central location to monitor and control a widely distributed network of sensors and to activate process connected to the 
network. SCADA has two major components - Remote Terminal Unit (RTU) and Master Terminal Unit (MTU). Issues related to the communication between the field component of SCADA - Remote Terminal Unit - and the central control station component - Master Terminal Unit - are discussed referring specifically to the radio communication. What factors that influence the selection of a SCADA package are then analyzed.

The regulatory authorities are gradually accepting monitored natural attenuation (MNA) as a legitimate approach for remediating contaminated groundwater plumes. But before an MNA scenario gets approved two conditions generally must be applied. A source reduction technology must be applied to eliminate or reduce the supply of contaminant held in soil pores released to the plume, and also an extensive monitoring program must be implemented to ensure that the predicted natural decay of the contaminants meets regulatory permit limits. Optimization of long-term monitoring systems for MNA is detailed.

Different types of monitoring strategies are suggested and all are compared against six performance criteria - cost, operability, reliability, durability, maintenance, and data quality. Of all the long-term monitoring strategies discussed in detail, two strategies are considered for the optimization. One of the strategies is a long-term monitoring system with network of multi-probe sensors. Multi-probe sensors are a few sensors in a unit that measure indicator or surrogate parameters; a few of these systems are commercially available. There are many commercially available data acquisition systems to select from. Selection of these systems depends on desired complexity. A second strategy is a LTM system with contaminant specific (special) sensors. There are different types of sensors available and a few of the more promising of them are 
discussed relative to their advantages and disadvantages. They are compared using the performance criteria and two of them are close to performance necessary for LTM systems acceptance by regulatory agencies.

Finally two different scenarios are discussed where these LTM systems are used and then trade-off analysis is done for both systems.

In the design and optimization of long-term monitoring systems for contaminated environmental sites, the use of either multi-probe or contaminant specific - special sensor systems, including data acquisition system / SCADA and its components have been shown to offer significant performance advantages over the baseline monitoring approach of well sampling and lab analysis.

The cost analysis shows that the multi-probe sensor approach offers significant cost reduction over the baseline sampling approach. With the multi-probe sensor approach and for a period of 30 years, for one well about $\$ 238 \mathrm{~K}$ of cost savings can be achieved and for a particular application of Savannah River site with approximately fifteen hundred wells about $\$ 350 \mathrm{M}$ of cost savings can be achieved, which is about 66 percent of cost savings.

Recommendations for further studies and developments might include (1) developing concepts for networking sensor arrays, (2) field testing actual monitoring strategies to evaluate their feasibility under actual field conditions, e.g., limitations on telemetry due to site topography, and (3) field testing of actual monitoring systems to evaluate actual data quality achieved and maintenance issues under longer term operating conditions. 


\subsection{Background-Contaminated Sites and Need for LTM}

The purpose of monitoring hazardous waste sites is to a) characterize soil and groundwater pollution in sufficient detail to facilitate site remediation, b) assess movement of contamination undergoing long-term remediation, c) assess movement of contamination posing little risk and requiring future remediation, and d) monitoring residual contamination left behind permanently. Site investigation involves the measurement of the physical, chemical and biological parameters that control subsurface contaminant transport at a given site. Geologic, hydrologic and chemical data, once collected and integrated, can be used to define the nature and extent of soil and groundwater contamination and the potential for migration of contaminants within the aquifer [1].

Monitoring is carried out to know the state of environmental pollution, propose a suitable protective action and assess the effectiveness of that action.

Environmental monitoring systems involve multiple tasks

- Identifying sources of substances that adversely effect the environment

- Determining the transport of these pollutants through the environment

- Measuring their concentration in the environment

Long-term monitoring (LTM) associated with subsurface contamination sites can be an expensive endeavor. Most LTM requirements, however, are mandated by federal and state regulations. Primary costs associated with LTM are labor costs. Sample collection, storage, analysis and reporting can add a significant financial burden when extended over many years. 


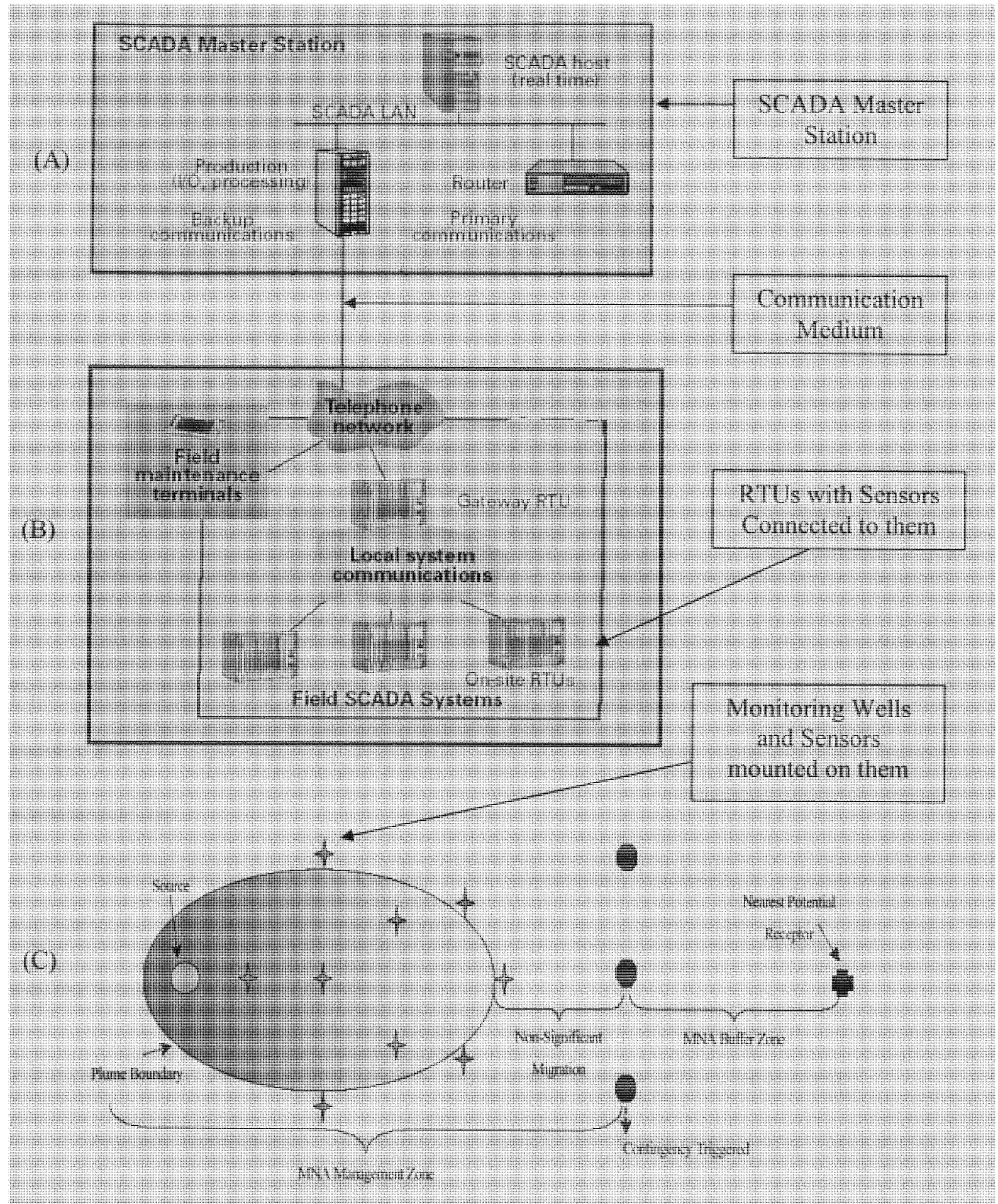

Figure 1: General Schematic of Long Term Monitoring System (Source: (A) (B) - http://www.isa.org/isaolp/journals/pdf/ic/991234.pdf

(C) - Technical guidance for the long-term monitoring of natural attenuation remedies at department of energy sites - Office of Environmental Restoration, October 8, 1999.) 
LTM costs can be significantly reduced through development of unattended, insitu monitoring networks of sensors capable of providing data satisfactory to regulatory concerns [2].

For many sites, monitoring usually begins with government-regulated groundwater sampling and analyses after a site has been determined to be contaminated and groundwater has been found to be affected. Once the nature of the contamination has been characterized, it becomes necessary to initiate clean up and verification that remediation objectives are being met through performance monitoring. This type of monitoring is referred as "process optimization monitoring." This monitoring is to ensure that remedial objectives are being met by tracking the progress of containment removal and to verify the physical integrity and continuity of a containment system as needed. Possible remedial actions can be grouped into four major categories: Containment, in situ stabilization, in situ removal, destination pumping \& ex situ treatment and natural attenuation [3].

After the contamination area has been remediated, stabilized, or contained, some type of long-term performance monitoring should be expected to continue for some years into the future [3].

\subsubsection{Differences - Process Optimization Monitoring and Long-Term Monitoring}

Process optimization monitoring is conducted during any active remediation phase, during which the contaminant concentration in the soil should be dropping. During this, more frequent sampling is required over a relatively short period of time. Since active remediation targets a specific region of contaminated soil, the spatial requirements 
for where to monitor are better defined. Furthermore, since the desired information will be used to control the process, detailed analyses with accuracy and precision will be required. In many cases, the soil, water, or gas samples are collected manually and samples are sent away for analysis. Because of the short time period over which process optimization monitoring is conducted, labor intensive, manual operations are a necessity.

On the other hand, long-term monitoring is expected to continue for years. Data quality requirements may be more relaxed than for process optimization monitoring. Also accuracy and precision are not of much concern. Only the changes need to be monitored. As these changes develop slowly, less frequent sampling over a longer time period is required. If the long-term monitoring data suggests that there is a problem that needs further investigation, then it is needed to go back to characterization and, perhaps, remediation.

Due to longer time periods, long-term monitoring is considered to be very costly if traditional methods are used. Sometimes the projected total life cycle cost of long-term monitoring exceeds the initial characterization and restoration costs [3].

\subsubsection{Vadose Zone:}

By textbook definition, "The Vadose zone is the geologic media between land surface and the regional water table". Two processes control water in the vadose zone. The first is gravity that moves the surface-applied water downwards. The second is the capillary process that moves the water in all directions and stores and releases it. A significant challenge of vadose zone science is to understand and describe the distribution and movement of water and contaminants within this subsurface environment. 
The vadose zone is typically the first subsurface environment encountered by contaminants. All subsequent groundwater and surface water concentrations are influenced by the complexities in this dynamic system. So the final goal of vadose zone science is to predict contaminant fate and transport behavior so that affected facilities can be managed safely, and then to identify better cost-effective options for cleaning up contaminants before they reach the groundwater. If the Vadose zone is monitored properly, opportunities for sensitive, early warning of potential environmental problems can be created [3].

\subsubsection{Characterizing Contamination}

In traditional groundwater monitoring, three types of wells - background, plume and down gradient - are installed and a few more for confidence may be added. The wells are sampled periodically and then sent for offsite analysis. Government regulators use the information obtained from this analysis to find out the potential impact on human health and the environment, and then to think of further characterization needs and potential eventual remediation needs of the site accordingly.

\subsubsection{Reducing Long-Term Monitoring Costs}

The key for reducing long-term monitoring costs is to significantly reduce active onsite sampling activities and related analytical costs, most likely through other passive approaches. These systems should be highly automated. They must collect data; transmit the information to a central location for final analysis, and provide storage that is accessible on demand. A high degree of automation can be achieved through a combination of sensors for continuous point measurements, and periodic geophysical 
measurements, to provide the necessary information for data interpretation. It is predicted that highly automated long-term vadose zone and groundwater monitoring systems will result in life-cycle cost reductions of one to two orders of magnitude over present traditional methods based on manual groundwater sampling [5].

Many sensors have been developed that can be used in the above decided systems. Advanced electrochemical, fiber-optic sensors, radiation micro-sensors, and masssensitive piezoelectric or acoustic wave transducers are some that are readily available.

For example such sensors can be deployed in a groundwater well to allow continuous unattended analysis of the water for contaminants of concern, and then only when the sensors indicate an increase in contaminant level, groundwater sampling would need to be conducted.

\subsubsection{Limitation of Sensors}

All sensors essentially make point measurements. Although there is a distinct advantage to making point measurements continuously in time, the inherent spatial discontinuity suggests that a dense grid of sensors may be required. Even if in the future the cost of sensors drops, the cost of placement will likely remain high; therefore, a dense grid of sensors is technically and economically attractive. The synergistic coupling of point sensor measurements with spatially distributed geophysical measurements is a way to achieve requirements using fewer point measurement sensors.

\subsubsection{Placing, Replacing and Calibrating Sensors}

Sensor installation is a vital issue in long-term monitoring, especially in the vadose zone. Larger, permanent vadose zone wells can be justified if some or all the 
sensors in the well are designed to be retrievable. These wells are expensive to install, but the cost savings from sensor replacement may eventually offset the cost of the well. An alternative is to use a lower cost placement technology and install all sensors permanently. If a sensor fails, it must then be abandoned and a new sensor placed. Or low-cost sensors can be deployed in redundant numbers, and as one sensor fails, another can be brought online [3].

\subsection{Long-Term Monitoring System Design}

Long-term monitoring system consists of a measurement system, communication system and a data acquisition system. Following sections discuss in brief design of longterm monitoring system and its major components.

\subsubsection{Measurement System}

Design of a cost effective long-term monitoring system is possible through the combination of point measurement sensors and spatially distributed geophysical measurements. Geophysical data, collected approximately annually, can be used to meet the spatial requirements of monitoring so that the density of point measurements can be reduced. Data from point sensor systems can be collected automatically at a frequency that meets the temporal requirement. If groundwater monitoring is required, data from groundwater sensor systems can be collected automatically and used to justify drastically reducing groundwater-sampling frequency (to about once every 5 years). 


\subsubsection{System Assembly}

In long-term monitoring, each group of sensors connected to data acquisition systems may collect gigabytes of data. In order to be useful, this raw data must be analyzed and transformed into information from which decisions can be made. As long as parameters to be monitored remain within acceptable bounds, all that is needed are average values along with some basic statistics like minimum, maximum and standard deviation. Data can be automatically transmitted to a central location by different ways of communication.

It is possible to set up a data storage buffer that contains the most recent detailed data set for a fixed interval of time. In the event of an alarm condition, the data in this buffer could be transmitted and analyzed to determine the details of the situation leading to the alarm. In this manner, the amount of data transmitted is minimized and the process is automated to the greatest extent possible. Automation is the key to minimizing lifecycle costs of long-term monitoring.

\subsection{Thesis Objectives and the Research}

This study proposes that optimized long-term monitoring systems should be deployed and operated autonomously for extended periods of time (at least a few years) on post-closure, contaminated sites to meet the objectives of LTM in remediation. These systems telemetry data to a central location using certain communication protocols, usually radiofrequency or cell phone technology. It is important that the data collected is analyzed and converted to information with various security levels of access, and can appropriately be made available to public, scientists, managers, and environmental 
regulators. All of this is technologically available today, but not generally in commercialoff-the-shelf (COTS) system. It is important that any optimal design follows the many federal and DOE standards (e.g., records management, data security protocols) and be designed to allow widespread use at various sites, with only slight modifications for sitespecific conditions and requirements.

The objective of this thesis is to provide the basis for the above proposal. Following sections briefly describe the theoretical perspective of long-term monitoring, the study approach for the thesis, and results expected of the thesis.

The first section in the second chapter talks about the sensors and issues about sensors, indicator or surrogate parameters, and second section talks about different types of environmental monitoring systems in use like data loggers and SCADA.

In third chapter long-term monitoring systems for monitored natural attenuation and their optimization are discussed. Two different strategies are suggested for optimization of long-term monitoring system and then optimization of LTM is discussed in case of two specific applications. Conclusion and recommendations follow that.

\subsection{Theoretical Perspective - Component Design of LTM}

Long-term monitoring Systems are composed of the following major components:

- Sensors - These elements collect the data. Sensors may need data verification/validation and calibration capabilities to facilitate acceptance of the data by various users. 
- Remote Terminal Station - These elements collect data from an array of sensors, and telemetries or transmit the data to some central location using a communication system (e.g., radio wave or cell phone technology)

- Central Control Station - The Central Control Station analyzes the data collected from all the sensors in the field and turns them into information. Information might be simply displayed in real time on the Internet or it may be used in a decision system to effect some change in the field systems. Analysis of data might lead to change in the frequency of data collection from certain sensors, might change remediation system parameters, or set off local alarms, etc. This system will store data in a database that can be viewed at will.

To monitor contaminants for long-term monitoring programs, point chemical sensors can be used, but there is an uncertainty regarding the ability of point chemical measurements to provide an accurate assessment of site conditions, even with numerous measurements. Instead of chemical sensors, another approach is to use sensors that monitor contaminants or chemical activity in the subsurface that provide indirect information about the fate and transport of the contaminant. Monitoring such subsurface "indicator" parameters (moisture, $\mathrm{pH}$, oxygen reduction potential, etc.) can provide critical information that may be a better monitor of the progress of subsurface processes, and an indicator of potential early system failure.

There are different commercial Supervisory Control Data Acquisition Systems (SCADAs) that consist of the Remote Terminal Unit (RTU), Telemetry System and Central Control Station. Trade-off studies have been conducted to determine those few systems that both meet monitoring goal requirements and offer the widest application. 


\subsection{Study Approach}

For all the major components of the Long-Term Monitoring System, there are advanced techonologies available today. This study will present some of the available technologies for each of the potential components in any long-term monitoring system.

Capabilities and limitations of various commercial Supervisory Control and Data Acquisition Systems (SCADA) will be documented in detail. This study includes suggesting optimal solutions for each component in a LTM system as well as the overall LTM system.

\subsection{Results of Research}

This will identify some of the major issues related to long-term monitoring systems, including those related to sensors, communications, security, and data acceptability. The state of the art among the various components will be detailed with benefits and limitations identified for all. The trade-off analyses for determining the optimal system design will be documented. A set of LTM scenarios will be presented and the optmized LTM system is presented. The cost analysis for different approaches will also be presensted.

\subsection{Needs and Gaps in LTM}

Most contaminant sensor systems require further study with regard to their usefulness and long-term stability in long-term monitoring systems. Also questions, such as operability and maintenance, how long between calibrations, and if in situ calibration is possible, must also be addressed. 
It is required to study integrated systems and optimize monitoring combinations that meet all the monitoring requirements. A system engineering approach must consider incompatibilities of various integrated systems and optimize logistics of collecting the data. 


\section{CHAPTER 2 - LTM SYSTEM COMPONENTS}

\section{SECTION I}

\subsection{Sensors}

For Long-term monitoring programs, a broad range of surveillance, monitoring, and data collection techniques need to be employed. One of the data subsets commonly used is contaminant concentration level in the subsurface zones. These levels are typically obtained by collecting samples from drilled monitoring wells on a periodic basis, and then these samples are sent to laboratories for standard analysis. High priority for site managers is to reduce the cost of these routine-monitoring analyses and to avoid drilling additional wells [10]. Possibilities for reducing monitoring costs include,

1. Use of field measurement technologies, and

2. Automation of sampling and measuring of concentrations in the field, with either field analytical methods or sensors [10].

In-situ sensors that measure contaminants reliably over extended periods of time automatically can be used for long-term monitoring. Numerous sensor systems have recently been developed that can supply this kind of information. Some of the most important ones are - advance electrochemical, fiber-optic, and radiation micro-sensors, or mass sensitive piezoelectric or acoustic wave transducers [3]. 


\subsubsection{Typical Contaminants of Concern}

During the last decade, over 1200 hazardous waste sites in the US have been placed on the EPA National Priority List for remedial investigation and cleanup. There are lots of contaminants that are cause of concern. Following are the contaminants of most concern [34].

Radionuclide contaminants: e.g. uranium, technetium-99, tritium, cesium-137, plutonium Metal contaminants: e.g. mercury, hexavalent chromium, lead.

Volatile organic contaminants: e.g. PCE, TCE, dichloroethylene, carbon tetrachloride [10].

The focus of my thesis though is on the Volatile Organic Compounds (VOCs) that pose a significant threat to groundwater supplies and are commonly detected in groundwater.

\subsubsection{Sensors used for Volatile Organic Compounds}

There are four major types of sensors for in-situ monitoring subsurface contaminants: A) Chromatography and Spectrometry; B) Electrochemical Sensors; C) Mass Sensors; D) Optical Sensors [33]

A. Chromatography and Spectrometry: Chromatography is a method for separation and analysis of complex mixtures of volatile organic and inorganic compounds. A chromatograph is essentially a highly efficient apparatus for separating a complex mixture into individual components. When a mixture of components is injected into a 
chromatograph equipped with an appropriate column, the components travel down the column at different rates and therefore reach the end of the column at different times. A detector is positioned at the end of the column to quantify the concentrations of individual components of the mixture being eluted from the column [33].

Bench-Top Gas Chromatographs: It can provide superior discrimination capabilities relative to other sensors with excellent precision, sensitivity and reproducibility. But it is not portable, and it is very costly.

Manufacturer: Agilent 6890 Price: In the range of $\$ 20000$ to $\$ 50000$ [33]

Portable Gas Chromatographs: It is portable and reliable. It has remote monitoring capability. It has part per billion ( $\mathrm{ppb}$ ) level sensitivity to vapors. It can detect wide range of volatile and semi-volatile components. It cannot be used in-situ. It is most likely very expensive.

Manufacturers: Femtoscan, HAPSITE, Bruker-Daltronics Inc.[33]

\section{B. Electrochemical Sensors:}

There are different kinds of sensors that can directly detect the contaminants and provide the required data to monitor the contaminant concentration in the groundwater. Chemiresistor Arrays:

Micro-chemical sensors are under development at Sandia National Laboratories, which will reduce the need for labor-intensive manual sampling for environment by establishing a system that constantly monitors for contaminants in soil and water. The heart of the system is an array of tiny sensors that can detect different volatile organic compounds. A sensor, which is called chemiresistor, is a polymer-absorption device fabricated by dissolving commercial polymers in a solvent with conductive carbon 
particles. The ink-like fluid is deposited and dried on wire-like electrodes on a specially designed microcircuit. When contaminants such as Volatile Organic Compounds are present, they absorb into the polymers. This causes polymer swelling, which changes the electrical resistance of the material. These resistance changes can be measured and recorded. They can be used to calculate the concentration of a contaminant in the vapor that is contact with the polymer. The polymers shrink if the chemical or contaminant of interest is removed, causing the material to revert to its original state and electrical resistance.

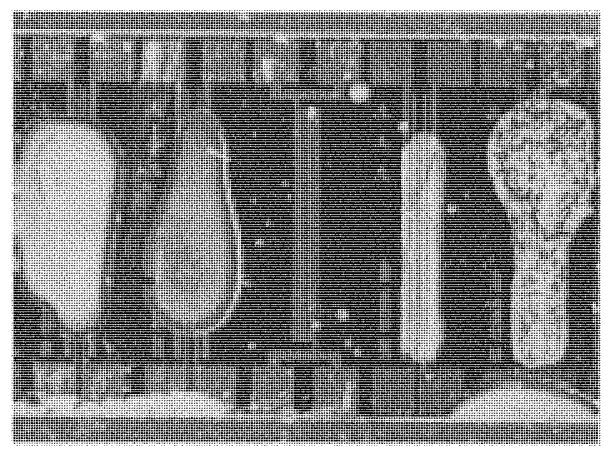

Figure 2: Chemiresistor array developed at Sandia National Laboratories (Source: Clifford K. Ho, Michael T. Itamura, Michael Kelley, and Robert C. Hughes, "Review of Chemical Sensors for In-Situ Monitoring of Volatile Contaminants", Sandia Report (SAND2001-0643) March 2001, page 14)

A package is also developed that can be used in water or underground. The package allows the chemiresistors to be exposed to contaminants in both gas and liquid phases. Inside the package the chemiresistor array chip is placed on a 16-pin dual inline package connected to a long weatherproof cable. The cable can be connected to any data logger. Measurements are made with DC voltages allowing cables to be almost any length. 
These sensors may not be able to replicate sampling method quality but improvements can be made that can help it achieve that. Currently improvements can be made in how sensors react to humidity, temperature and pressure changes. Also research can be done about how the various polymers in the chemiresistors will react to unknown chemicals.

Advantages: Chemiresistors are small, low power devices that have no moving parts and have good sensitivity to various chemicals. Another advantage in comparison to standard electrochemical sensor is that chemiresistors don't require liquid water to work properly.

Disadvantages: They may not be able to discriminate among unknown mixture of chemicals. Durability of polymers in subsurface environments is uncertain and robust packaging need to be developed. Although the process of measurement is reversible, signal may experience hysteresis and shift in the baseline when exposed to chemicals [33].

Manufacturers: Cyrano Sciences (Price - Around \$7000), Adsistor Technology (Around $\$ 50-60$ ), Sandia National Laboratory [33].

Catalytic Bead Sensors:

They are used in the detection of methane in the air. Catalytic bead sensor is comprised of a passive and active element, both made from an embedded coiled platinum wire in porous ceramic. The active element is coated with a catalyst such as platinum, and the passive element is coated with an inert glass to act as a reference element to compensate for the environmental conditions. Both elements are heated to a prescribed operating temperature ranging from $300^{\circ} \mathrm{C}$ to $800^{\circ} \mathrm{C}$. When a combustible gas such as 
methane contacts the elements, the vapor combusts on the active element and the active element increases in temperature. As a result, the resistance of the platinum coils changes. The two elements are connected to a Wheatstone bridge circuit, so the changes in resistance are measured as a change in voltage.

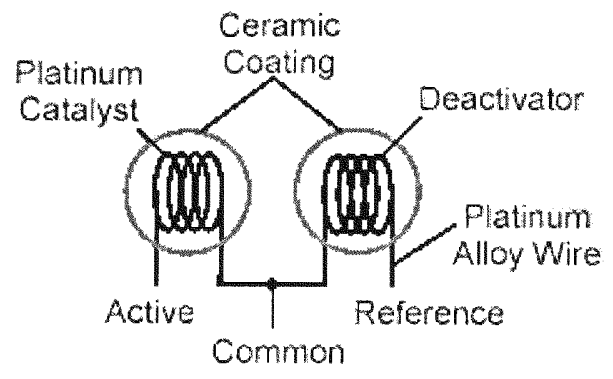

Figure 3: Catalytic Bead Sensor

(Source: Clifford K. Ho, Michael T. Itamura, Michael Kelley, and Robert C. Hughes, "Review of Chemical Sensors for In-Situ Monitoring of Volatile Contaminants", Sandia Report (SAND2001-0643) March 2001, page 15)

Advantages: The units are very portable.

Disadvantages: The units are not amenable for in-situ monitoring. It requires elevated temperatures for operation. Internal pump is required to sample the gas.

Manufacturer: GasTech (Price - \$2000) [33]

Metal-Oxide Semiconductor Sensors:

Advantages: These sensors have a high sensitivity to combustible gases. They are compact and durable. They are relatively expensive.

Disadvantages: The sensor has a fair amount of sensitivity to water humidity, which may be problematic in subsurface environments.

Manufacturer: Figaro 


\section{Mass Sensors:}

Surface Acoustic Wave Sensors/Portable Acoustic Wave Sensors:

Surface acoustic wave sensors (SAWS) are small miniature sensors used to detect VOCs. A SAW device consists of an input transducer, a chemical absorbent film, and an output transducer on a piezoelectric substrate like quartz. The input transducer launches an acoustic wave that travels through the chemical film and is detected by the output transducer. The device runs at a very high frequency $100 \mathrm{MHz}$. The velocity and attenuation of the signal are sensitive to the mass of the thin film that can allow for the identification of the contaminant. A signal pattern recognition system that uses a clustering technique is needed to identify various chemicals [33].

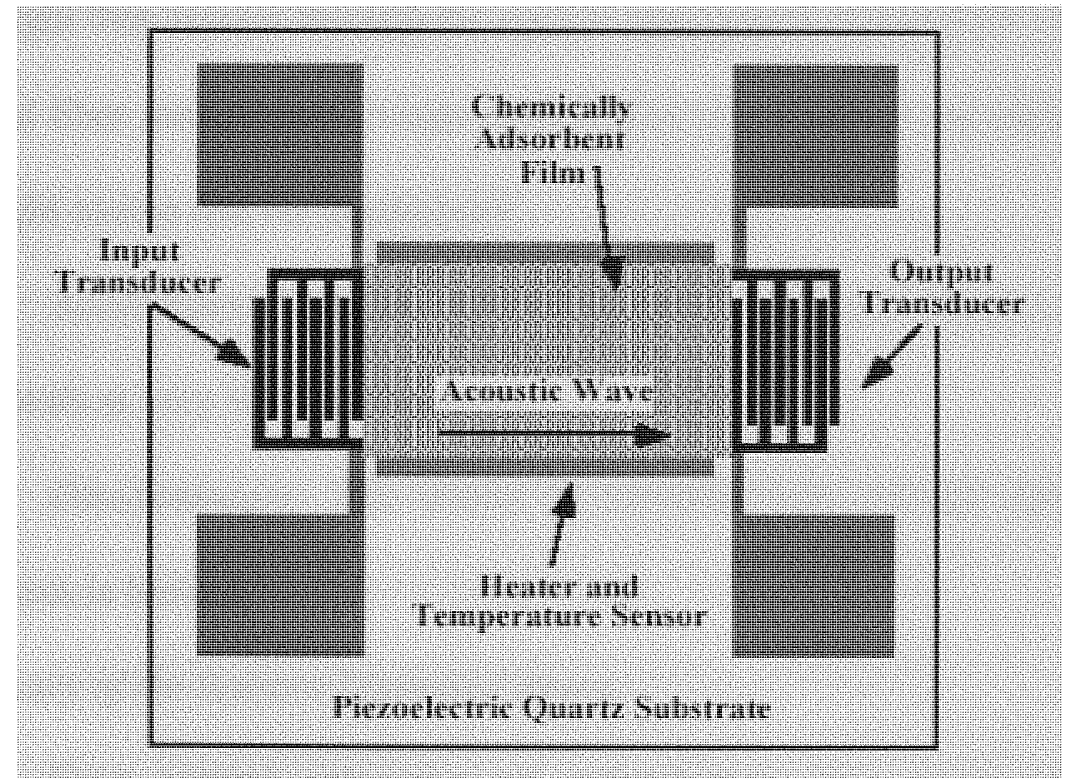

Figure 4: Schematic of Surface Acoustic Wave Sensor

(Source: Clifford K. Ho, Michael T. Itamura, Michael Kelley, and Robert C. Hughes, "Review of Chemical Sensors for In-Situ Monitoring of Volatile Contaminants", Sandia Report (SAND2001-0643) March 2001, page 19) 
Advantages: It is small, requires low power, and has no moving parts other than high frequency excitation. It can detect chemicals in very low concentrations.

Disadvantages: It may not be able to discriminate among unknown mixtures of chemicals. Some polymers react strongly to water vapor. It has uncertain durability in subsurface environments.

Manufacturer: Sandia National Laboratories

D. Optical Sensors:

Fiber Optic Sensors:

Fiber optic sensors are a class of sensors that use optical fibers to detect chemical contaminants. Light is generated by a light source and is sent through an optical fiber. The light then returns through the optical fiber and is captured by a photo detector. Some optical fiber sensors use a single optical fiber while others use separate optical fibers for the light source and for the detector.

Advantages: It has low power requirements, no moving parts. It can detect various chemicals at very low concentrations.

Disadvantages: The ability to transmit light through optical fiber over long distances is limited.

Summary of the Sensors Discussed Above:

Four general categories of technologies were reviewed for their potential application in real-time, in-situ chemical sensing applications. The first category reviewed was chromatography/spectrometry. The gas chromatographs reviewed provide excellent discrimination among various chemicals of interest. 


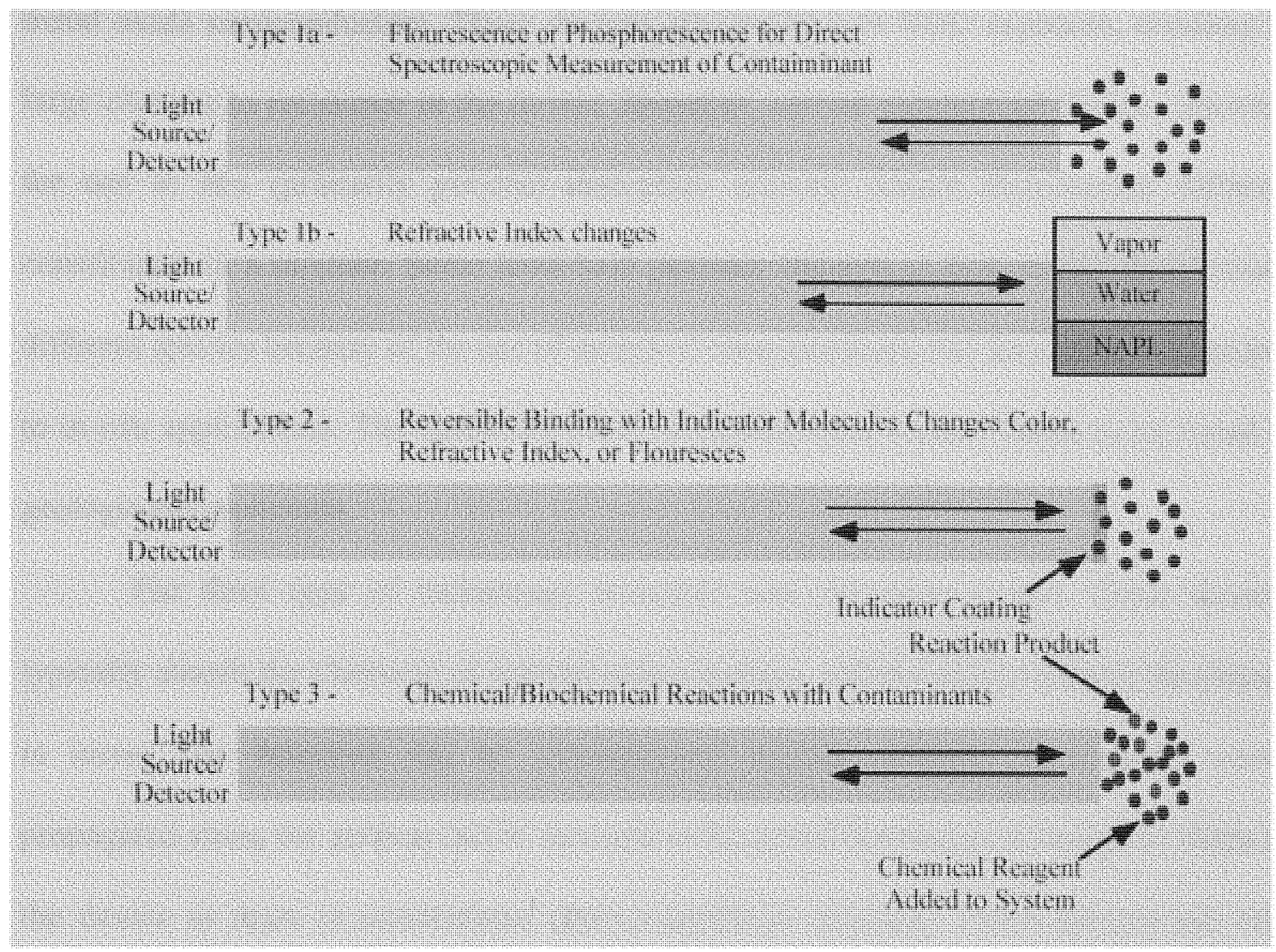

Figure 5: Fiber Optic Sensor (Three different types)

(Source: Clifford K. Ho, Michael T. Itamura, Michael Kelley, and Robert C. Hughes, "Review of Chemical Sensors for In-Situ Monitoring of Volatile Contaminants", Sandia Report (SAND2001-0643) March 2001, page 22)

The sizes of gas chromatographs range from large bench-top systems to portable hand-held systems and microchips the size of a coin. Although the portable GCs can be taken to the field and used manually to sample monitoring wells, the majority of these devices are not yet amenable to real-time, in-situ applications. The $\mu$ ChemLab might be a potential candidate, but it requires micro-pumps to circulate gas through the system, and these moving parts may not be able to withstand long periods in geologic environments.

The second category reviewed was electrochemical sensors. The conductometric sensors reviewed include polymer-absorption chemiresistors, catalytic bead sensors, and 
metal oxide semiconductors. These devices are sensitive to VOC exposure, resulting in large changes to resistance in the device. However, current commercial devices intended for use in situ (primarily polymer-absorption sensors) cannot discriminate different constituents in a mixture. Some hand-held polymer absorption devices can discriminate different species because of the use of arrays of chemiresistors, but they are not amenable to in-situ applications. The durability of all electrochemical sensors needs to be tested in geologic environments.

The third type of technology reviewed was the mass sensor. These devices typically absorb the chemical of interest onto a surface, and the device detects the change in mass. The detection can be accomplished through changes in acoustic waves propagated along the surface (SAW devices) or by actual bending or a change in shape of the device as mass is accumulated (micro-cantilever devices). These devices are very sensitive to the presence of various volatile and semi-volatile chemicals. However, the sensitivity of the device depends on high-frequency excitation or extremely small changes in shape; these aspects have not been tested in prolonged geologic environments.

Finally, the fourth category of devices reviewed was optical sensors. These include fiber optical sensors. These sensors rely on changes in electromagnetic radiation to detect and identify the presence of chemicals. The sensitivity of these sensors to VOCs can be good, and a TCE fiber optical sensor integrated with a cone penetrometer already exists. Its use in long-term applications still requires testing. The devices reviewed were not amenable for real-time, in-situ applications involving other lower volatility VOCs.

The most viable sensors for in-situ chemical sensing appear to be electrochemical sensors, specifically conductometric sensors, based on their simplicity and robustness. 
Reports from the U.S. EPA $(1992,1995)$ have indicated that polymer-absorption and metal-oxide-semiconductor sensors are viable candidates for use at underground storage tanks. In addition, fiber-optic sensors and mass sensors (SAW devices in particular) also appear to be viable candidates for in-situ applications. The general issue among all of these sensors is that few, if any, have been tested and demonstrated in long-term geologic environments. In addition, the data obtained from these sensors is not typically quantified (e.g. what chemical species are present? How much is present? Where is it located?) [33]. Table 1: Contaminant Specific Sensors w.r.t. Performance Criteria*

\begin{tabular}{|c|l|l|l|l|l|l|l|}
\hline $\begin{array}{c}\text { Sr } \\
\text { No }\end{array}$ & Sensor Type & Cost & Operability & Reliability & $\begin{array}{l}\text { Durabil } \\
\text { ity }\end{array}$ & Maintenance & $\begin{array}{l}\text { Data } \\
\text { Quality }\end{array}$ \\
\hline 1 & $\begin{array}{l}\text { Electrochemical } \\
\text { Sensors }\end{array}$ & & & & & & \\
\hline 1.1 & $\begin{array}{l}\text { Polymer- } \\
\text { Absorption } \\
\text { Chemiresistors }\end{array}$ & Low & High & Medium & Low & Medium & High \\
\hline 1.2 & $\begin{array}{l}\text { Metal-Oxide } \\
\text { Semiconductor }\end{array}$ & High & High & Medium & Medium & Medium & High \\
\hline 2 & Mass Sensors & Medium & High & Medium & Low & Medium & High \\
\hline 2.1 & $\begin{array}{l}\text { Surface Acoustic } \\
\text { Wave Sensors }\end{array}$ & Medium & Medium & Medium & Medium & Medium & High \\
\hline 3 & Optical Sensors & Medium & & & \\
\hline
\end{tabular}

* Details about performance criteria are discussed in the section 3.3.2

\subsubsection{Moisture Data}

To identify best ways to detect early warning signs of system failures for engineered isolation facilities such as landfills, and their caps is a significant issue for environmental remediation projects. Monitoring the moisture content and flux is emerging as a baseline monitoring approach regarding DOE engineered facilities [10]. Because moisture sensors are commercially available, many site managers would like to focus on developing integrated systems that monitor moisture flux, water content, and 
soil water potential below and around remedial systems. The monitored moisture data not only give an early indication of potential system failure, but also facilitate specific site understanding of the transport pathways and processes that influence contaminant movement.

Two examples of existing environmental sensor systems are considered highly potential formats for use in integrating newly developed sensors into LTM programs. The first example is found in a U.S. Geological Service nationwide stream/river monitoring program, in which certain measurements are automatically taken remotely on a periodic basis, with the data transmitted via telemetry to a central database. The data are then accessible to stakeholders through the Internet. Standard commercial sensors and data acquisition systems are already in place in many applications. DOE could leverage its resources by using these established systems as the 'backbone' for monitoring systems and adapting the sensors as required for environmental applications [10].

The second example is the E-SMART® network technology developed during the last decade by General Atomics with the support of the Air Force Research Laboratory, Defense Advanced Research Projects Agency, U.S. General Services Administration, and DOE [10]. The system employs a standard networking protocol capable of connecting sensors from various manufacturers into a single network for device monitoring, data logging, and peer-to-peer communications. One E-SMART $®$ system in operation polls more than 100 sensor variables at ten-minute intervals. Variables include water level, pH, conductivity, dissolved oxygen, oxidation-reduction potential, turbidity, temperature, barometric pressure, humidity, wind speed, wind direction, rainfall, differential pressure, $\mathrm{AC}$ current, and total volatile organics (hydrocarbons and halocarbons, e.g., BTEXs, 
TCE, and PCE). Some signals are received directly via hardwire, while others are received via radio communication from remote sites (including sites at which sensors and radios are powered by solar panels). More information is available from the General Atomics website (http://www.ga.com/atg/html/environ.html) [10].

\subsection{Issues about Sensors and Field Analytical Methods}

The main concerns about sensors for contaminants are whether they can be selfcalibrated and stay calibrated for long periods of time, and whether they can be reliable over years of operation. In light of the significant investment required to overcome these concerns, alternatives to sensors might better serve monitoring programs. In addition, automated sampling combined with advanced field methods may offer more opportunity for productive change in monitoring protocols [10]. Furthermore sensors could be more advantageously used in several ways:

1. Be inexpensive, and placed in numerous positions where previously only one data point was captured,

2. Be designed to change out easily upon failure,

3. Reduce the sampling waste created in monitoring programs,

4. Be placed in difficult-to-reach locations and possibly eliminate exposure to contaminated medium for field workers previously collecting samples.

\subsection{Issues related particularly to Sensors}

Low-cost robust sensors are needed for remote, in-situ monitoring of contaminants and verification of remedial actions. Subsurface sensing targets include contaminants such as organics, metals, radionuclides, water quality indicators such as 
salinity, $\mathrm{pH}$, oxidation-reduction potential, etc., soil characteristics and radiation levels. Some commercially available sensors can meet the general functional requirements for sensing these parameters in soil or groundwater, but are not suited to remote, in situ operation with automated data transmission.

Site-specific constraints, such as the size of a borehole or depth to the water table, are the chief obstacles to the deployment of existing sensors. Harsh operating conditions and variable contaminant levels also present challenges for the deployment and operation of sensors for long-term monitoring (LTM) applications [16].

When selecting sensors for a given application, factors such as measurement frequency, sensitivity, selectivity, depth of deployment, and life cycle cost must be considered. Additionally, real-time, continuous operation or the ability to take periodic measurements may be needed. Customer, regulatory, and stakeholder requirements determine the sensor performance characteristics and impose constraints upon the operation and emplacement of the sensor packages. The sensors must be sensitive enough to meet detection limits required by State and Federal regulators. Instruments designed for other applications (such as, characterization of high-level waste) may not have the required sensitivity to detect contaminants at the ppb or ppt level and usually require frequent calibration [16].

The depth of deployment is an issue because the length of coaxial cables or fiber optics required to reach groundwater could cause signal degradation, especially in the western U.S. where the depth to the water table may be more than $500 \mathrm{ft}$ at some sites. In such cases, a signal booster may be necessary. 
The costs of equipment (sensors, data loggers, telemetry system, etc.), installation, operation and maintenance, data collection, and data transfer for the LTM system must provide a cost savings over traditional monitoring schemes such as periodic manual sampling, laboratory analysis, and disposal of investigation-derived waste. The higher initial start-up cost of installing a sensor system needs to be balanced against the potential long term cost savings based on the life expectancy of the LTM sensor system. If the site is heavily contaminated, environmental, safety and health issues will impact the installation cost of the sensor system.

The sensor package must have the ability to withstand harsh environments and provide reliable data over the expected range of environmental conditions and events, including temperature, radiation, $\mathrm{pH}$, humidity, lightning strikes, etc. For example, the operating temperature range in the vadose zone is $-20^{\circ} \mathrm{C}$ to $45^{\circ} \mathrm{C}$, for groundwater it is $2^{\circ} \mathrm{C}$ to $30^{\circ} \mathrm{C}$, and for instrumentation above ground it is $-45^{\circ} \mathrm{C}$ to $150^{\circ} \mathrm{C}$. How long the sensor will last in corrosive media or in harsh chemical or high radiation environments is a key concern. In such instances, the sensor(s) may need to be sealed within an inert package and should be capable of maintaining functionality, calibration, and accuracy for years without replacement or repair, since the sensors are intended to be left in place for extended periods with little or no maintenance.

Smart sensors can serve as sentinels to trigger an alarm if a threshold value is exceeded or a sensor malfunction is detected. In the event of a sensor malfunction, the sensor should be retractable for servicing or replacement. The sensor package must operate remotely with data stored and downloaded by telemetry to a centrally located processing station. The remoteness and topology of the waste sites can cause power 
supply and communication problems. The sensor package should have low power requirements, with power provided by batteries or a renewable energy source, such as solar power, for remote site monitoring. A stable and reliable power source must be available for the telemetry system to ensure the transmission covers the expected distances for deployment [16].

The physical size requirements for sensors are determined by the available methods of sensor emplacement. For subsurface monitoring using existing boreholes or monitoring wells, the sensor package should typically have a diameter of less than 4 inches, and in some cases, less than 2 inches. Length should be less than 4 feet for monitoring in wells. For vadose zone monitoring, sensor configurations need to be shorter than a foot. For new boreholes, the diameter of the sensor package is limited to inside diameter of emplacement technique (e.g., direct push). It must be ensured that the sensor package is protected from mechanical damage or vibration during insertion and positioned at the correct depth [16].

Regulatory acceptance of the data generated by LTM sensors is critical to their utilization. Field-testing is essential prior to full-scale deployment for all interested parties to evaluate the acceptability of the data if the sensors deployed are new and comparative data is lacking. Data obtained from sensors deployed for LTM of VOCs, metals, or radionuclides must be compared to data derived using currently accepted methodologies [16].

One of the alternatives is to design sensors systems for monitoring indicator* parameters such as moisture; $\mathrm{pH}$ or oxygen levels and that might be more easily achievable. The indicator parameter measurements might be better indicators of early 
system failures than direct contaminant measurements. Also monitoring indirect parameters can provide critical information about the condition and integrity of a remedial system.

* - Also called bulk parameters; indirect approach to monitoring the Fate \&Transport of the contaminants of concern

2.5 Indicator Parameters: Example of LTM for Monitored Natural Attenuation (MNA)

Long-Term Monitoring systems require real-time, in-situ sensors that measure contaminants up to certain concentration levels reliably over extended periods. However designing sensors and integrated sensor systems for monitoring alternative or surrogate parameters that are good indicators of remedial system performance might be easily achievable. Indicator parameters might be better indicators of early system failures than actual contaminant measurements. As an example, indicator parameters for monitoring MNA processes are addressed in the table and graphs below. MNA - Monitored Natural Attenuation - is basically the reliance on natural attenuation processes that reduce mass, toxicity, volume or concentration of the contaminants in the groundwater to achieve the remedial objectives. Some of the natural processes are biodegradation, dispersion, and dilution. Many chlorinated contaminants can be destroyed by biodegradation. Detailed discussion about MNA is in the section 3.1.

Table 2 describes each indicator parameter, its meaningful range and that range reflects. 
Table 2. Examples of the Indicator Parameters for MNA process activity

\begin{tabular}{|c|c|c|}
\hline Parameter & Meaningful range & What it reflects \\
\hline Chloride & $>2 \mathrm{x}$ background & $\begin{array}{l}\text { Daughter product of organic chlorine } \\
\text { being generated }\end{array}$ \\
\hline Dissolved Oxygen & $<0.5 \mathrm{mg} / \mathrm{L}$ & $\begin{array}{l}\text { Suppresses reductive dechlorination at } \\
\text { higher concentrations }\end{array}$ \\
\hline Dissolved Oxygen & $>1 \mathrm{mg} / \mathrm{L}$ & $\begin{array}{l}\text { Vinyl chloride may be oxidized } \\
\text { aerobically, but reductive dechlorination } \\
\text { will not occur at higher DO levels }\end{array}$ \\
\hline Nitrate & $<1 \mathrm{mg} / \mathrm{L}$ & $\begin{array}{l}\text { May compete with reductive pathway at } \\
\text { higher concentration }\end{array}$ \\
\hline $\begin{array}{l}\text { Oxidation- } \\
\text { Reduction } \\
\text { Potential }\end{array}$ & $\begin{array}{l}<50 \mathrm{mV} \text { against } \\
\mathrm{Ag} / \mathrm{AgCl}\end{array}$ & Reductive pathway possible \\
\hline $\mathrm{PH}$ & $5<\mathrm{pH}<9$ & $\begin{array}{l}\text { Tolerated range for reductive pathway; } \\
\text { outside these boundaries anaerobic } \\
\text { dechlorination may not happen }\end{array}$ \\
\hline Sulfate & $<20 \mathrm{mg} / \mathrm{L}$ & $\begin{array}{l}\text { May compete with reductive pathway at } \\
\text { higher concentration }\end{array}$ \\
\hline
\end{tabular}




\subsubsection{Trend Graphs - Examples}

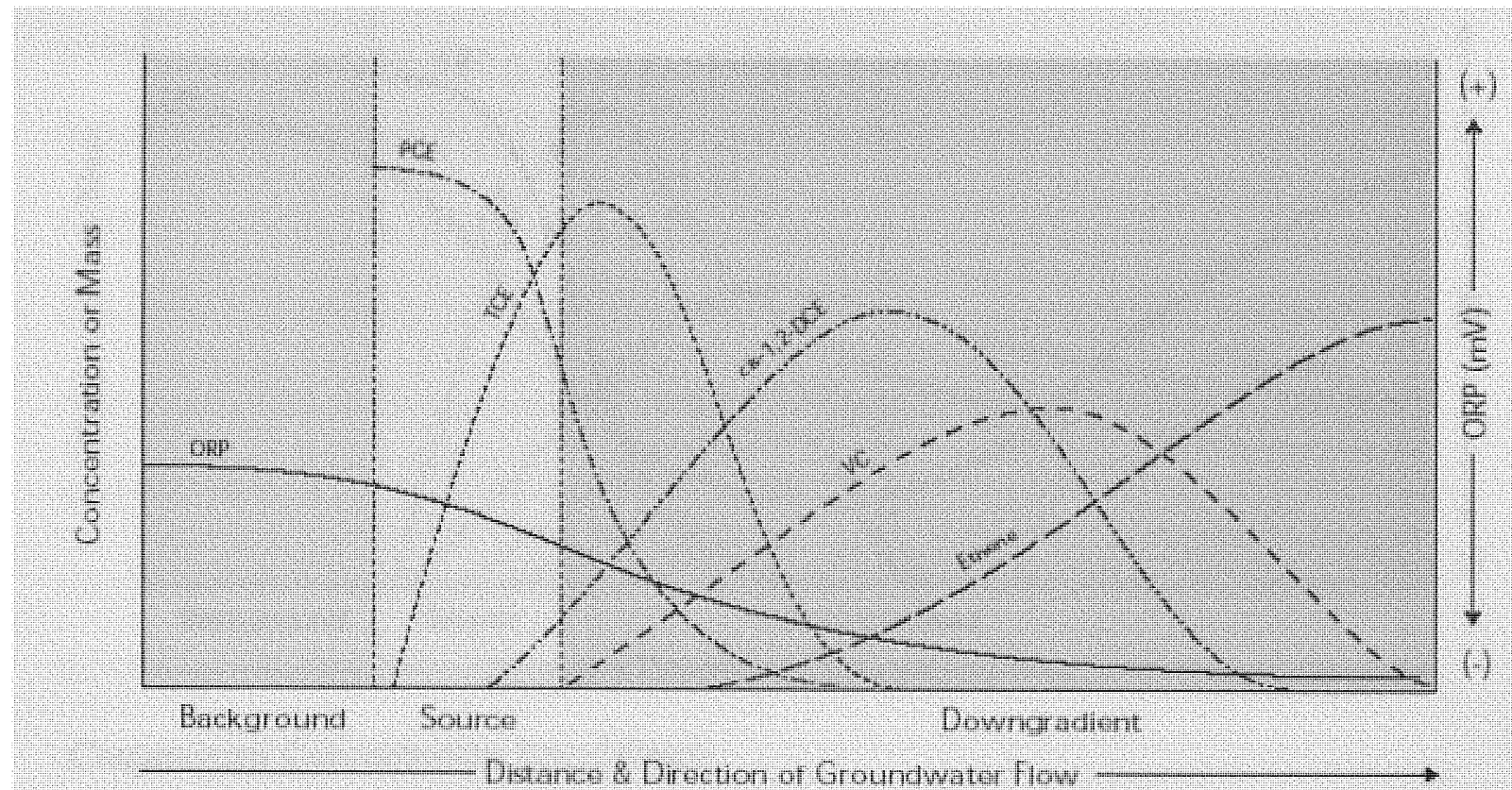

Contaminant Concentration Trend Graph

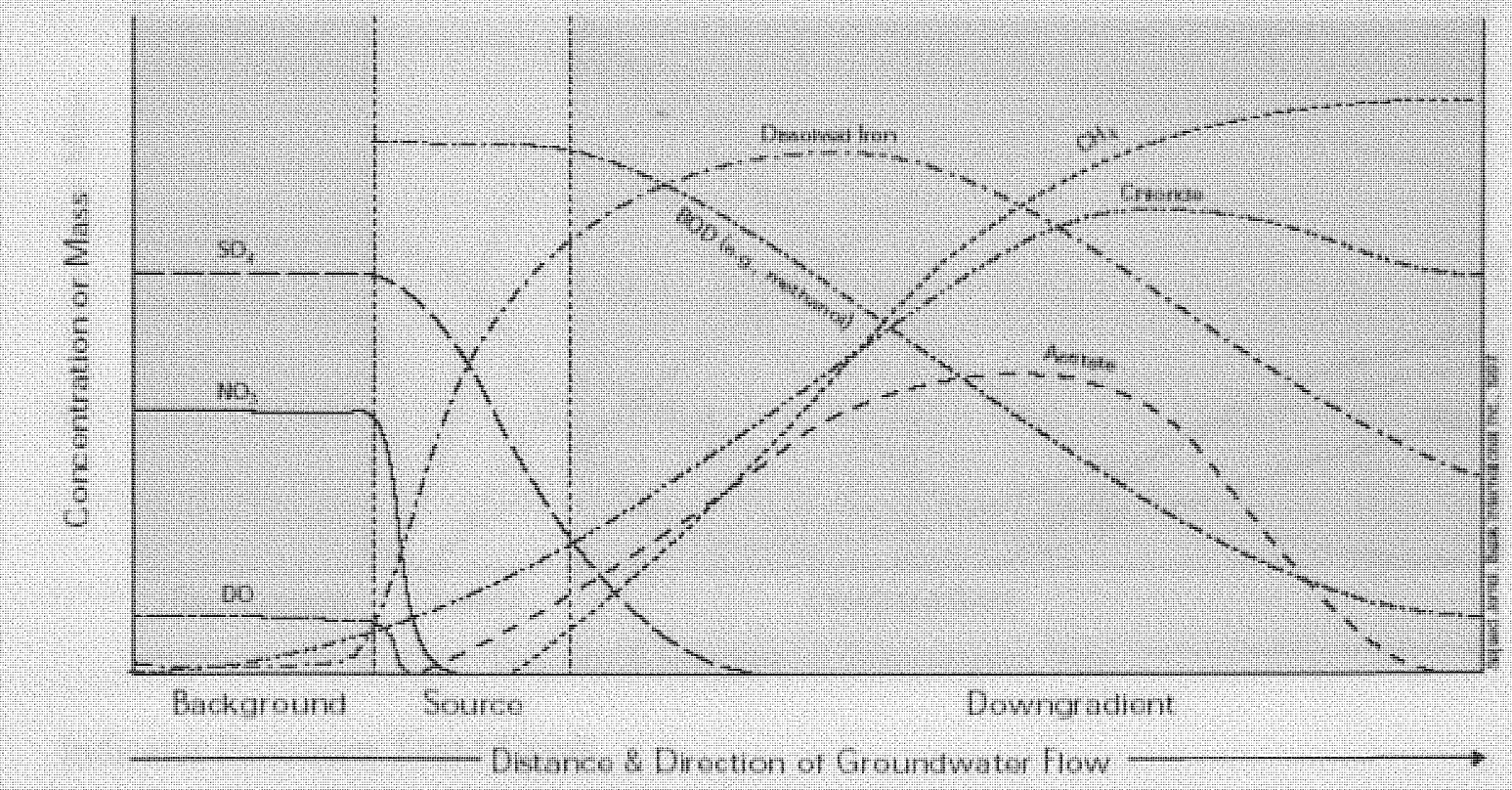

Indicator Parameter Trend Graph

Figure 6: Common Patterns of Chlorinated Solvent Biodegradation in an anaerobic system [32]. 


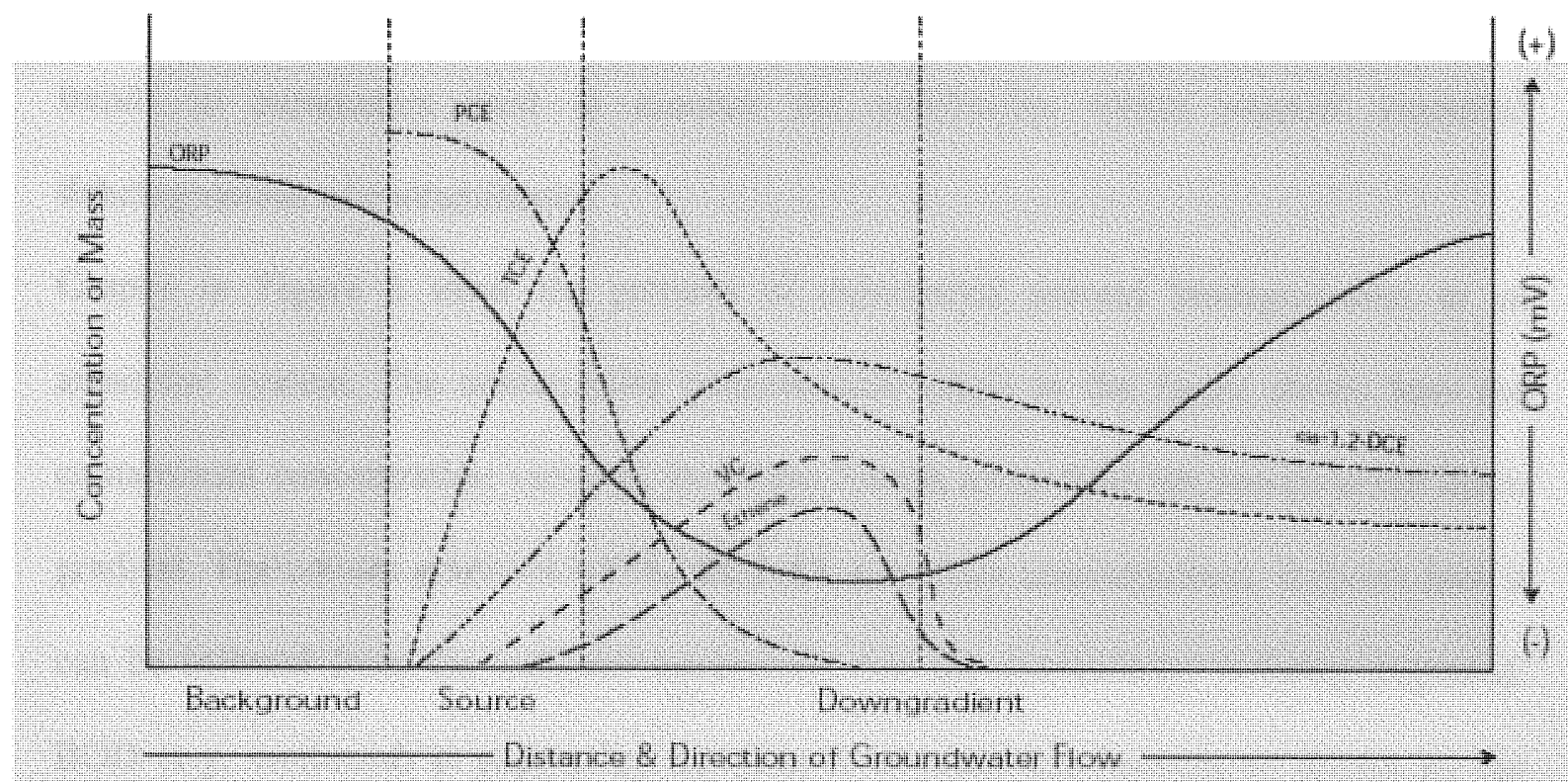

Contaminant Concentration Trend Graph

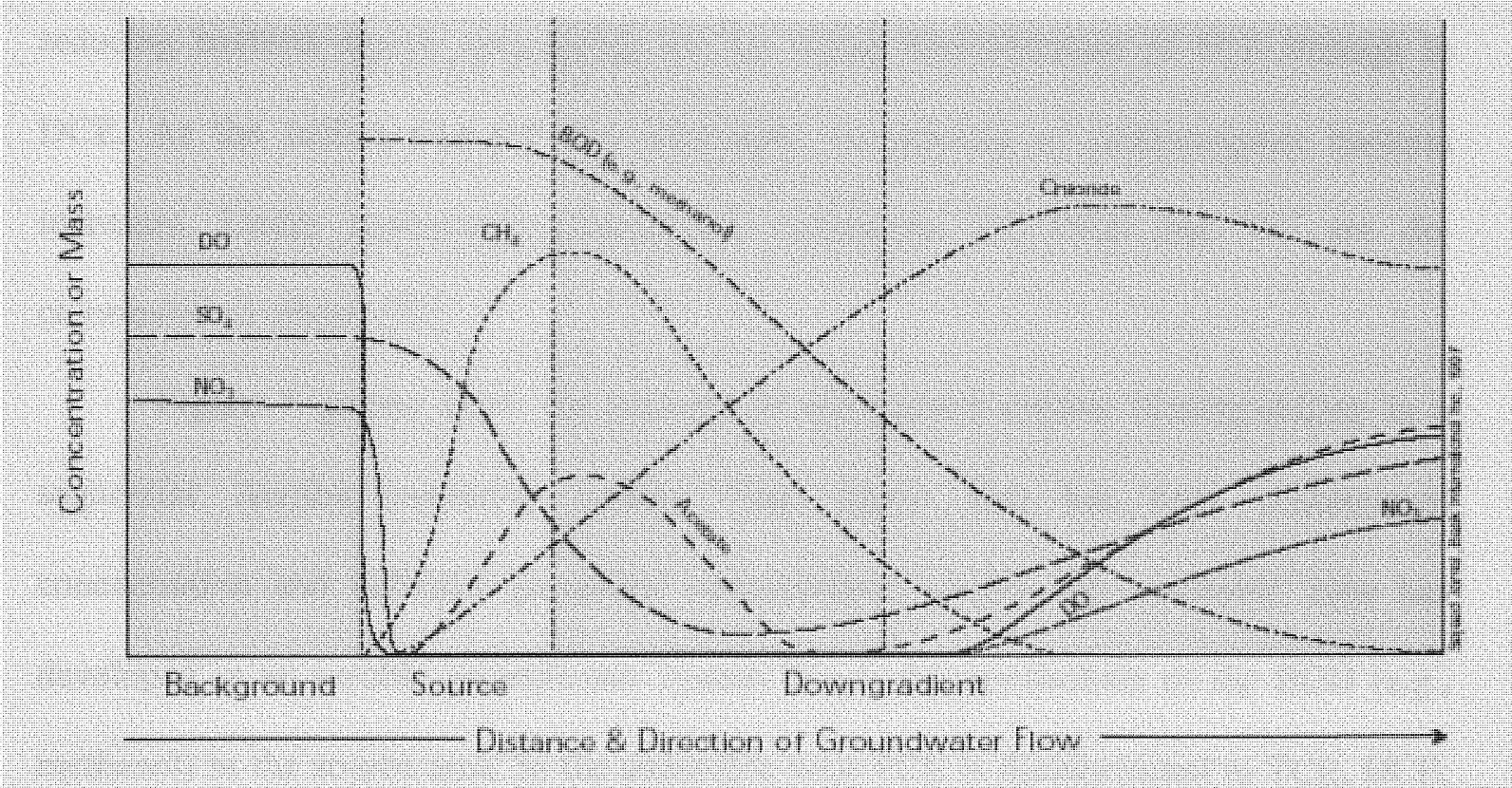

Indicator Parameter Trend Graph

Figure 7: Common Patems of Chlorinated Solven Diodegradation in a Sequental Aerobic/Anaerobic System [32]. 
These graphs compare the trends in degradation of chlorinated contaminants with the trends in parameters used to monitor the conditions, which support anaerobic dehalogenation of contaminants.

Graphs in Figure 6 show the patterns of chlorinated solvent biodegradation in an anaerobic system. The contaminant concentration graph in the figure shows how the contaminant concentration varies as the plume travels away from the source to the down gradient. The bottom graph shows the indicator parameter concentrations. It shows how it can be inferred from the indicator parameters that the contaminant is being degraded. BOD (e.g. methanol) is supporting the growth of anaerobic bacteria as shown by the production of methane and acetate and depletion of sulfate. Sulfate-reducing and ironreducing bacteria appear responsible for the initial dechlorination of PCE through to DCE. As the sulfate concentration decreases, the activity of methanogenic bacteria increases. Under methanogenic/acetogenic conditions 1,2 DCE and VC are dechlorinated to ethene [32].

Figure 7 graphs show the patterns of chlorinated solvent biodegradation in a sequential Aerobic/Anaerobic system. That figure also has both contaminant concentration and indicator parameter concentration graphs. An anaerobic zone has developed in an aerobic groundwater system due to the metabolism of the BOD (e.g., methanol) in the source area. In the anaerobic zone PCE is dechlorinated to TCE, DCE, VC and finally ethene. Methanogenic, sulphate-reducing, iron-reducing, and acetogenic bacteria are active, and their interactions are responsible for the dechlorination. However, the dechlorination rate is insufficient to cause all of the TCE and DCE to be dechlorinated in the anaerobic zone. These chemicals along with methane, ethane and 
vinyl chloride migrate into the transition and aerobic zones. In the transition zone the TCE and DCE is partially cometabolized by methanotrophs growing on the methane. Ethene and $\mathrm{VC}$ are mineralized to $\mathrm{CO} 2$ by aerobic bacteria in the aerobic zone [32].

\subsubsection{Generic Scenario:}

This section describes the general scenario of a contaminated groundwater system. The figure below shows the scenario in detail.

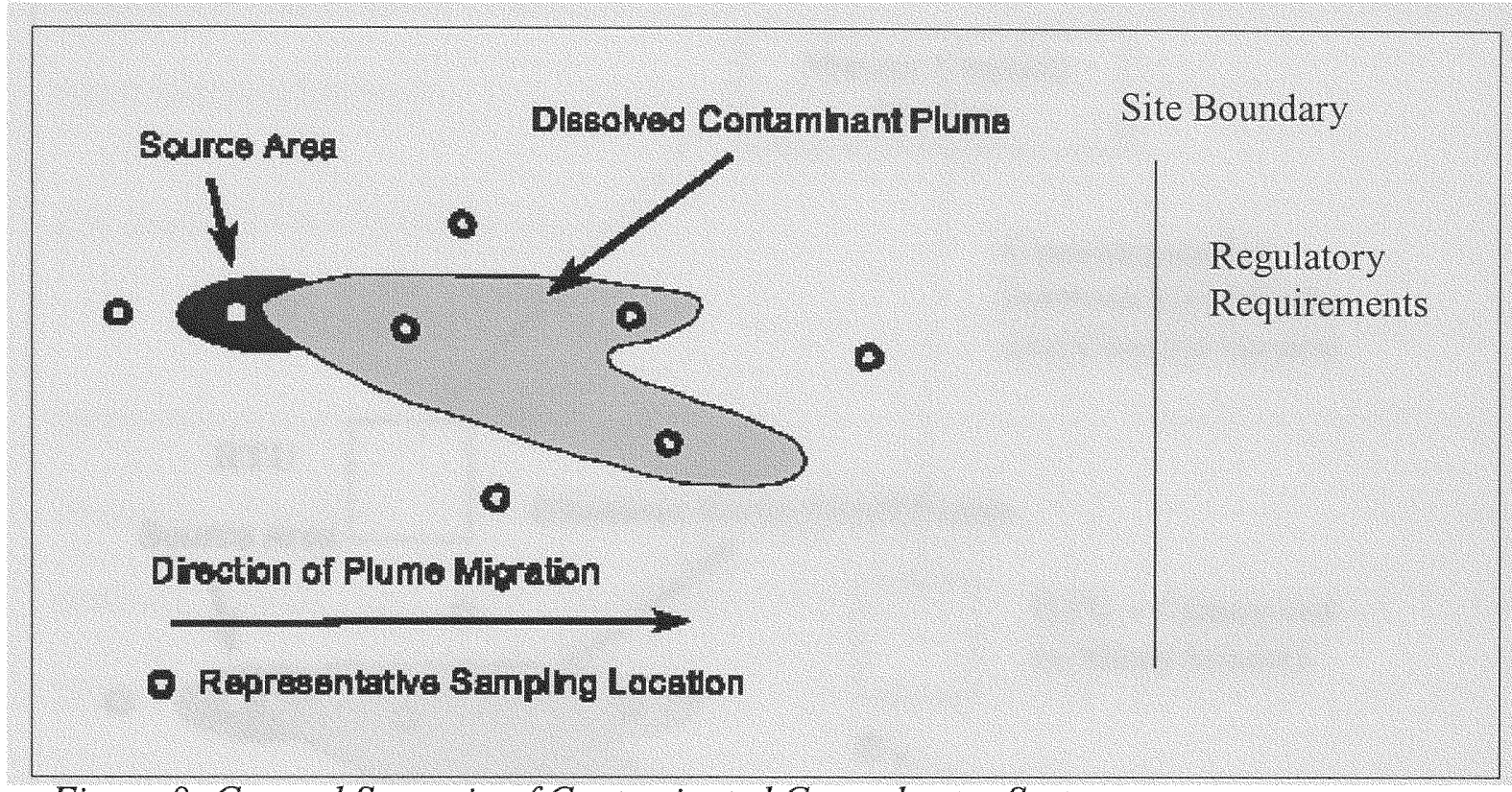

Figure 8: General Scenario of Contaminated Groundwater System

(Source: United States Environmental Protection Agency "Technical protocol for evaluating natural attenuation of chlorinated solvents in groundwater" Sept 1998)

The monitoring wells are located as shown in the figure 8 to monitor the contaminant concentrations in the groundwater plume. The groundwater plume moves at certain groundwater movement velocity. At the site boundary, regulators define some requirements regarding what should be the contaminant concentration when the plume reaches that point. The velocity of the groundwater plume, the regulatory requirement for the contaminant concentration, distance between the source and the site boundary, current 
contaminant concentration, all influence the prediction of whether the actual contaminant concentration will be within the regulatory requirements when it reaches the site boundary. The contaminant concentration should be continuously monitored in order to be sure that it stays below the limits when it reaches that site boundary.

Long Term Monitoring (LTM) System for the generic scenario:

Following figure 9 shows a typical Long-Term monitoring system.

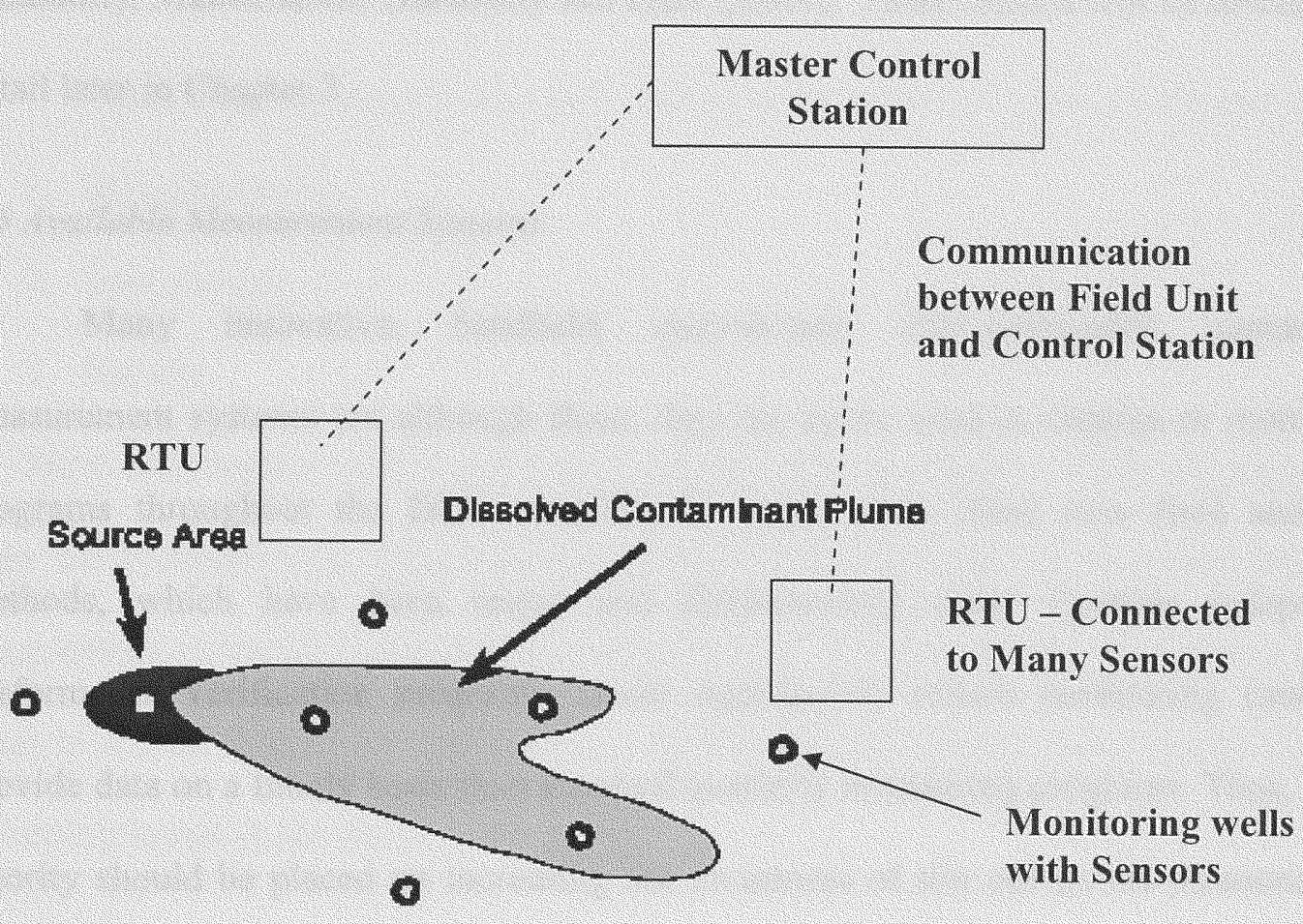

Direction of Plume Migration

- Representative Sampling Locstion

Figure 9: Typical Long Term Monitoring System

(Source: United States Environmental Protection Agency "Technical protocol for evaluating natural attenuation of chlorinated solvents in groundwater" Sept 1998) 
In a long-term monitoring system, as shown in the figure, there are sensors in the field, installed at the sampling locations, the Remote Terminal Units to which the sensors are connected, the communication link between the field units (RTU) and the Master Control Station. Optimization of the long-term monitoring system could be done by using optimized components. Comparison between the available technologies for each component considers the priority among the performance criteria - Cost, Operability, Reliability, Maintenance, Durability and Data Quality. These criteria will be discussed in detail later in Chapter 3.

\subsection{Available Measurement Systems}

Many innovative, handheld, easy-to-use, field-deployable contaminant measurement systems are although there, they are rarely used in cleanup or monitoring programs throughout the DOE complex. In many cases these new field analytical methods, which have been tested and demonstrated under rigorous independent performance verification programs, could significantly reduce monitoring costs and provide data on a timely basis than standard quarterly monitoring programs. Thus, a high priority should be placed on increasing the awareness of site operations personnel, site managers, and regulators about these methods and their potential uses [11]. 


\section{SECTION II - ENVIRONMENTAL MONITORING SYSTEM}

\subsection{Data Loggers}

Data logger is an electronic instrument that records measurements and events over time. The following sections discuss about the components of a typical data logger, its operation, and how it can be used as an environmental monitoring system.

\subsubsection{Data Logger Components:}

Typically, data loggers are small, battery-powered devices that are equipped with

1. Microprocessor,

2. Data storage

\section{Sensors.}

Most data loggers utilize software on a personal computer to initiate the logger and view the collected data. Data loggers are used in remote areas or anywhere where the convenience of battery power is desired to record measurements. Data loggers can be used in a wide variety of applications. Their small size makes data loggers ideal for mounting in out-of-the-way locations [17].

\subsubsection{Operation of a Data Logger}

The data logger is connected to a personal computer and the software is used to select the parameters such as sampling intervals, start time, etc. and to initiate the logger. The logger is then disconnected and deployed in the desired location. The logger records each measurement and stores it in memory along with the time and date. The logger is then reconnected to the personal computer and the software is used again to read-out the 
data and to view the measurements graphically, showing the measurements trending over time. Data in tabular form can be viewed or exported to a spreadsheet for further manipulation [17].

The low power requirements, long-term storage, and lack of emphasis on (supervisory) control distinguish these systems from SCADA systems.

\subsubsection{Data Loggers as part of Environmental Monitoring System}

Data loggers are typically used for environmental monitoring at remote sites. They are capable of some control actions, but the primary purpose of data loggers is to $\log$ values from a variety of sensor types with good reliability and accuracy, maintain that data for long periods, and operate on low power budgets.

\subsection{SCADA}

This section addresses topics including what is SCADA, what is SCADA comprised of, what the important applications of SCADA are, who makes them, what are the tradeoffs in using SCADA systems instead of other systems, and the security aspects that need to be considered.

\subsubsection{What is a SCADA?}

SCADA is the acronym for Supervisory Control and Data Acquisition and SCADA will be used for any further reference to these types of data acquisition systems. SCADA allows an operator at a central location to monitor and control a widely distributed process. It is most beneficial when a process occupies a large geographical area and instead of sending personnel on-site to take readings and make adjustments, 
monitoring and control of the entire system can be done from one central location. SCADA can store and retrieve historical data and also can provide comparisons of history with current process data. Standard PCs with windows-based graphical user interface can be used for the operator interface [18].

\subsubsection{SCADA Components}

The SCADA consists of a Remote Terminal Unit (RTU), which provides interface to the field analog and digital signals situated remotely, and a Master Terminal Unit (MTU) where information is displayed on the operator interface. The RTU communicates with the MTU through a communication link (radio, telephone, satellite or microwave). This technique used in transmitting and receiving information or data over a transmission medium is called telemetry. The relation between the MTU and RTU is analogous to master and slave.

\subsubsection{Master Terminal Unit:}

The MTU initiates all communication, gathers data, stores the information, and sends information to other systems and interfaces with the operator. See Figure No 10.

\subsubsection{Remote Terminal Unit:}

There are two types of RTUs, one type carries out measurements, transmits them to the MTU, and applies the commands it receives from the MTU. This type doesn't have decision-making capability. The central computer does the entire load of processing and scanning RTUs continuously. This is not a preferred category of RTU. 


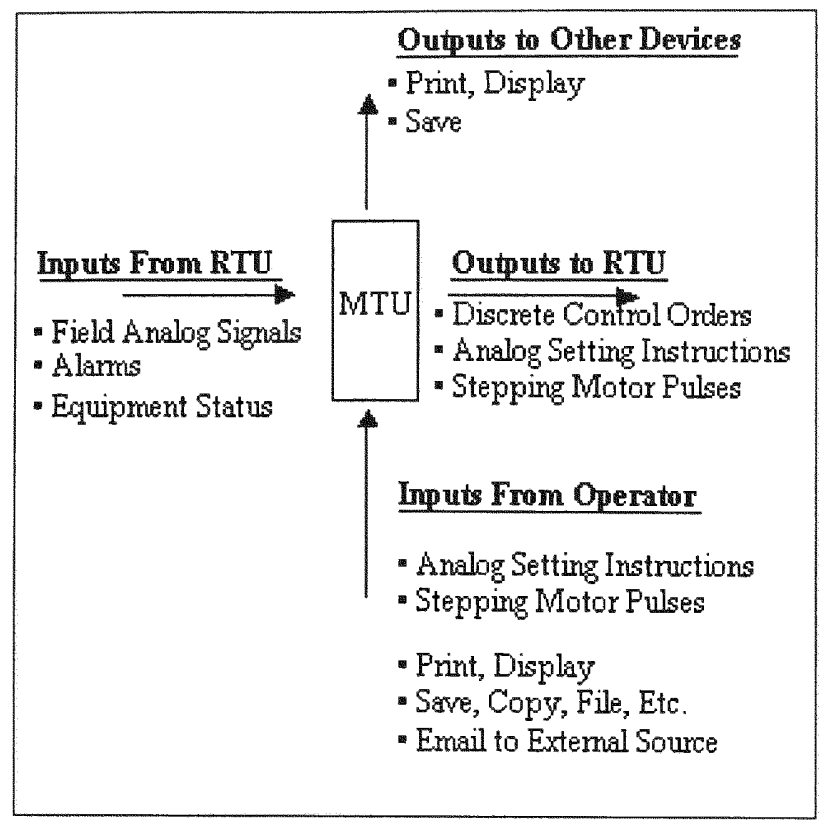

Figure 10: Master Terminal Unit - Interfaces to RTU and other devices (Source: http://www.riskinfo.com/cyberisk/Watersupply/SCADA-thesis.html)

The other type of RTU can make decisions on its own depending on the inputs, without consulting the MTU. This accelerates the overall system's reliability since it continues to operate even if MTU stops performing or the communication is lost. These aspects are presented in figure no. 11.

\subsubsection{Communication between RTU and MTU}

Telemetry is a technique used in transmitting and receiving information or data over a transmission medium. The information can be measurements, such as voltage, speed or flow. These data are transmitted to another location through a medium such as cable, telephone or radio frequency. Information may come from multiple locations. 


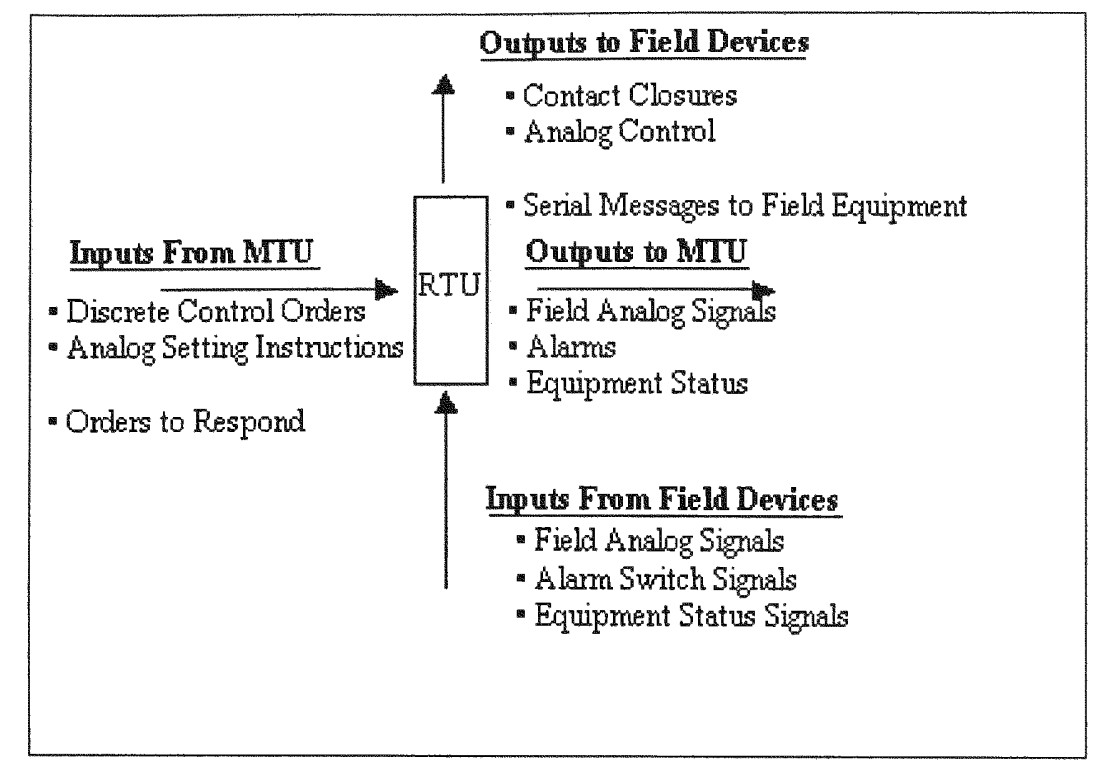

Figure 11: Remote Terminal Unit - Interfaces to MTU and other devices (Source: http://www.riskinfo.com/cyberisk/Watersupply/SCADA-thesis.html)

There are two typical network configurations for the wireless telemetry radiobased SCADA systems. They are the point-to-point and the point-to-multipoint configurations.

\section{Point-to-Point Configuration}

The Point-to-Point configuration is the simplest set-up for a telemetry system. Here data is exchanged between two stations. One station can be set up as the master and the other as the slave.

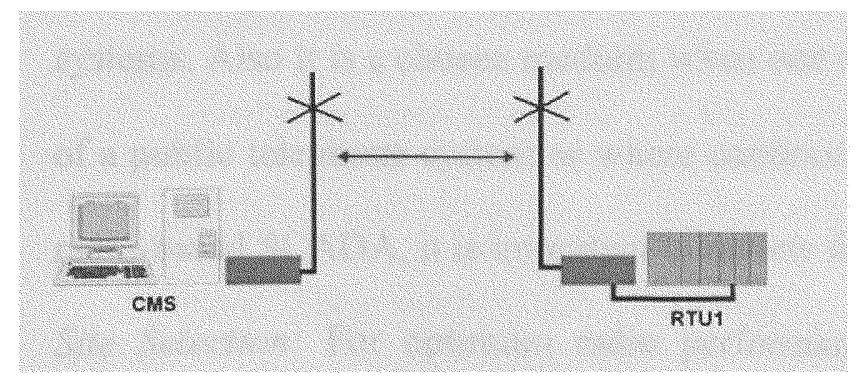

Figure 12: Point-to-Point Configuration (Source: http://www.micrologic-systems.com/primers/scada10.htm) 


\section{Point-to-Multipoint Configuration}

The Point-to-Multipoint configuration is where one device is designated as the master unit to several slave units. The master is usually the main host and is located at the control room, while the slaves are the remote units at the remote sites. Each slave is assigned a unique address or identification number.

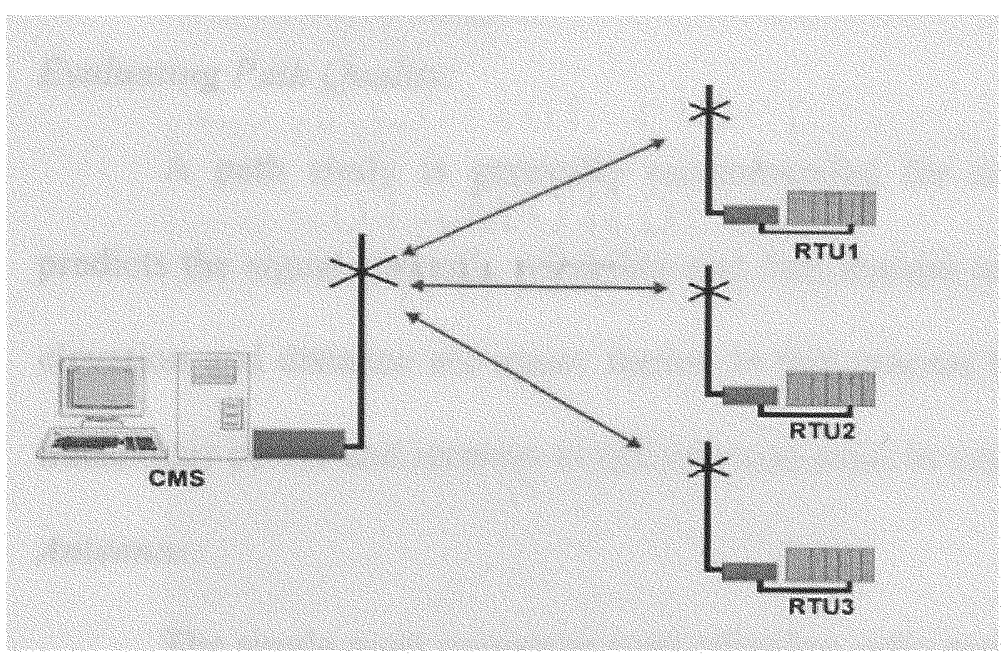

Figure 13: Point-to-Multipoint Configuration

(Source: http://www.micrologic-systems.com/primers/scada10.htm)

Providing a reliable, cost-effective communication method of transporting data is a critical element in realizing the full benefits of the SCADA system. While there are numerous methods available for transporting data, radio represents an attractive option to many people because it offers easy availability and gives the user direct control over their systems. Also it is a chosen medium when one or more sites are outside the coverage area of a public telephone system or where continuous data monitoring is required. In case of radio-based SCADA, it is important to review following parameters [12]:

Site Selection: For optimum radio performance the installation sites for master and remote stations must be carefully considered. Suitable sites should provide - 
- Protection of radio equipment from direct weather exposure

- Source of adequate and stable primary power

- Suitable entrances for antenna, interface or other required cabling

- Antenna location that provides unobstructed transmission path in the direction of the associated station [12].

Evaluating Path Quality:

A path study is generally recommended for new installations. A path study predicts the signal strength, reliability and fade margin of a proposed radio link. Terrain, elevation and distance are major factors in this process but antenna gain, feed line loss, transmitter power and receiver sensitivity also must be considered [12].

Antennas:

The single most important item affecting radio performance is the antenna system. High-quality gain antennas, designed specifically for use at the intended operating frequency, should be used at all master and remote stations. SCADA communication antennas fall into two general categories:

1. Omi-Directional Antenna - Since it provides equal radiation in all directions, it is appropriate for use at master stations, which communicate with an array of remote stations scattered in various directions. Figure 14 shows the two types of omni-directional antennas - client antennas and base station antennas.

2. Directional Antenna - At remote stations, a directional antenna such as yagi is used. They confine the transmission and reception to a relative narrow lobe allowing greater communication range and reducing the chance of interference to and from other users. Figure 15 shows the directional antennas. 


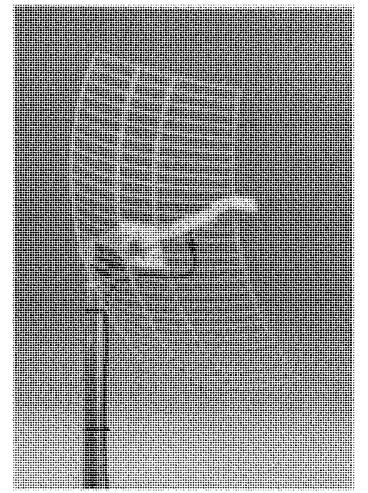

Client Antennas

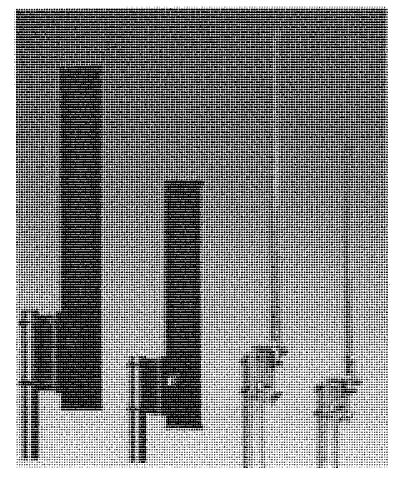

Base Station Antennas

Figure 14: Omni-Directional Antennas

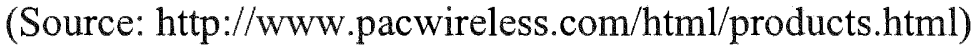

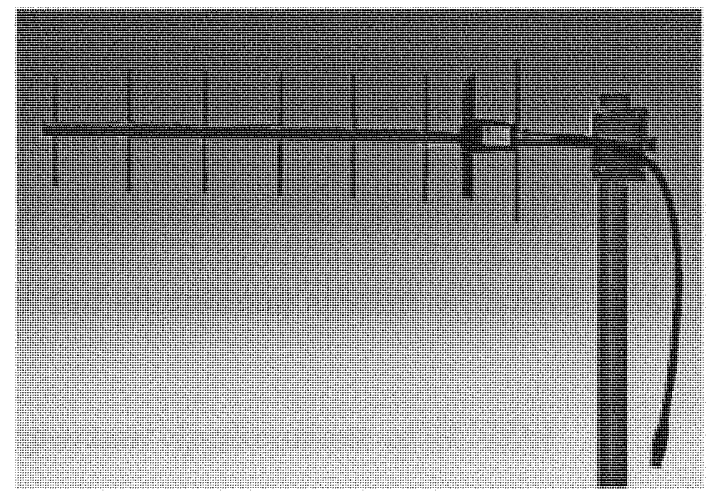

Figure 15: Yagi Antennas

(Source: http://www.pacwireless.com/html/yagi_900mhz.html)

The following recommendations apply to all antenna installations:

- Mount the antenna at a location that provides clear path in the direction of the associated station.

- Polarization of the antenna is important. Cross polarization between stations can cause a signal loss of $20 \mathrm{~dB}$ or more. 
Feedlines: It is very important to use low-loss antenna feedlines because the wrong cable can cause a huge reduction in efficiency, and these losses cannot be recovered with any amount of antenna gain or transmitter power.

Maintaining Performance: To ensure peak operating efficiency, preventive maintenance is required. Master and remote sites should be checked periodically to identify and correct problems. Antennas and feedlines should be inspected for physical damage. Spread Spectrum Technology:

Spread spectrum (SS) is one of the technologies applied to radio-based SCADA systems. SS uses a modulation technique that distributes a transmitter's signal over a wide bandwidth, making it virtually undetectable to a conventional receiver. Direct Sequence systems are commonly used in short-range SCADA applications. In the US, no license is required for $902-928 \mathrm{MHz}$ SS operation, with a maximum transmitter power of one watt and an antenna system with $6 \mathrm{~dB}$ gain [12].

\subsubsection{Important Applications of SCADA}

1. Water Industry:

- Brisbane City Council has installed a major water / waste water management SCADA network which currently consists of:

- Six hot standby base stations

- Approximately 15 supplementary base stations

- In excess of 300 Remote Terminal Units

The system employs report by exception and polling. 
- The Jordan Valley Water Conservancy District (JVWCD) recently installed a monitoring system that records water quality parameters of the culinary water supply before it enters the aquifer. The system consists of a CR10X data logger that monitors $\mathrm{pH}$, ORP, conductivity (total dissolved solids), and free chlorine. The data is passed to a PLC and then on to a central computer. A CR10X-based weather station monitors general weather parameters at the main treatment plant [21].

- Municipal water system - With a line of sight range over 20 miles, license free communications can be used for wide area SCADA communications either on a system wide basis or in conjunction with licensed radio system. In one application a water district used license free SCADALink as tail end extensions in a municipal SCADA communications systems, which used $900 \mathrm{MHz}$ licensed radio communications. The license free links are used to improve or enable coverage to sites obstructed by terrain and not within licensed RF coverage. License free links are used since it was not possible or difficult to get additional licensed frequencies for telemetry purposes in this dense urban environment [22].

In another application, a central PLC in the water treatment plant controlled and monitored 6 well pump sites, which were equipped with wireless RTUs. To reach remote sites outside of the 20-mile range of the wireless RTU, two back- to-back RTU's may be configured as a repeater to extend coverage [22]. 
2. Natural Gas Monitoring

3. Plant Facility Water Systems

4. Wastewater Treatment Plant

5. Irrigation Automation

6. Electrical Substation Monitoring and Control

7. Chemical Plant - Environmental Monitoring

8. Oil and Gas Industry

Commercial Vendors of SCADA:

[1] Geomation Measurement and Control Systems. [2] Motorola Inc.

[3] DATAC Control International Ltd.

[4] Measurement Systems Ltd.

[5] Campbell Scientific, Inc.

[6] Adroit Technologies

[7] $\mathrm{ABB}$

[8] Advanced Control Systems

[9] Automation \& Electronics, Inc.

[10] Automation Solutions.

[11] B\&R Industrial Automation Corp.

[12] Bentek Systems.

[13] The Foxboro Company, Invensys.

[14] C3-ilex, LLC.

[15] Horton Automation Inc.

[16] Intellution

[17] Moseley Associates.

[18] Yokogawa Inc.

Other Control Systems used

Apart from SCADA, there are a few other popular control systems used, including the following:

- Programmable Logic Controller Systems (PLC Systems)

- Distributed Control Systems (DCS)

- Building and Facility Management Systems (BMS/FMS) 
- Operator Interface Systems (OI)

2.8.5 Differences between Distributed Control Systems (DCS) and SCADA Systems:

A Distributed Control System, (DCS) is another type of control and monitoring system. Historically Distributed Control Systems use Programmable Logic Controllers (PLC) and SCADAs use RTU. PLCs have more intelligence capabilities than RTUs, but recently the difference has been minimized, and the lines between the two have blurred considerably.

The most important difference between a SCADA system and a DCS system is that SCADA systems can have irregular and incomplete reporting of field information but DCS systems are expected to have regular and complete I/O scans. DCS systems MUST poll ALL I/O within some given time constraint.

SCADA systems do not have any fixed period of time in which they complete an I/O scan. After issuing a control to the field, a SCADA system can raise the rate at which a remote station is polled for a more timely display of an expected change. Then after some event or at the operator's discretion, the polling rate can be reduced. DCS systems poll all the $\mathrm{I} / \mathrm{O}$ regardless of process changes.

Closed loop control is definitely not recommended for SCADA systems. SCADA systems do not guarantee polling of inputs at any rate. DCS systems are well suited to closed loop control. This guarantees polling at a given rate.

SCADA systems handle wide area networks of $\mathrm{I} / \mathrm{O}$, and provide overall supervisory data acquisition, alarming, and operator-commanded control. Due to the nature of SCADA, they tend to be asynchronous systems. This means that they send a lot 
of requests out to a lot of remote $\mathrm{V} / \mathrm{O}$ devices, and wait for responses. So SCADA systems are tolerant of non-responsive or slow to respond $\mathrm{I} / \mathrm{O}$ systems. To handle these remote communications with a high degree of security and limited communications bandwidth, SCADA protocols have developed over the years to handle a high degree of error detection. Common protocols today are DNP 3.0 (USA), IEC 870-101 (International). As SCADA systems access widely geographic areas, the Graphical User Interface (GUI) must be able to display geographic maps with functions such as panning, zooming, layering. World coordinate mapping and interface to GIS systems is also required.

DCS systems generally have localized $\mathrm{I} / \mathrm{O}$ with deterministic response times. Therefore these systems tend to be synchronous systems. They execute a control based on the input. They generally are not tolerant of failure to access data. DCS systems always provide some easy-to-configure method of defining control algorithms.

DCS systems are usually limited in distance to the $\mathrm{I} / \mathrm{O}$, new developments can extend the transmitters over fiber to kilometer ranges, but SCADA I/O can be located anywhere in the universe as long as there is a common channel of some type between the locations.

\subsubsection{Selection of the Appropriate SCADA Package}

There are many SCADA packages available on the market and the choice of the best available SCADA package requires the development of a detailed specification. It is very critical to identify who is going to use it. It is essential to make sure whether the package meets the alarm handling needs, the way alarms are displayed and are routed to the operator [13]. 
Flexibility of $\mathrm{I} / \mathrm{O}$ scanning is another important consideration. It should be possible to delay less important scans and allow high priority scans to proceed because this reduces the maximum load on communications. Another major technique for speeding up throughput is using event-driven systems that only report significant changes. This can reduce the loading of the communications by an order of magnitude. It is always a good idea though to do a complete scans of all I/O periodically [13].

Whatever system is chosen, initial hardware cost will represent part of the total system cost. Configuration is the most expensive aspect, so anything that simplifies it saves time and money. Maintenance costs are proportional to the cost and complexity of the original configuration. Selection of the operating system is also a very critical task [13].

\subsubsection{SCADA Operating Security}

SCADA systems are vulnerable to cyber attack by hackers and tampering. These assaults can damage the system substantially if it is not protected.

Risk:

SCADA systems are at great risk because more people are familiar with computers and also the systems. These are readily accessible as they use standard interfaces such as telephones. The things that are possible fall into two categories:

1. Accessing Information

2. Modifying settings 


\section{Secure Communication:}

There are a lot of techniques developed to ensure secure communication. The most important thing is the application of appropriate algorithms in a logical manner. This will make the access to communication medium or data hard, though not impossible.

The basic idea behind this is that making the message difficult to understand, i.e. unintelligible. That protects the message during the time it passes between the sender and receiver. Even if the message in intercepted it is worthless to the interceptor since it is encrypted and it has to be decrypted in order to read it.

Communication security has three major aspects; (1) ensuring privacy i.e. blocking access to transmitted information, (2) ensuring that the message is unaltered, and (3) authenticating the sender so that the system responds only authorized users. An Encryption Suite:

A number of algorithms are available for encryption, such as symmetric key algorithms and public key algorithms. Since no single algorithm can provide the desired result, the choice of three different algorithms is recommended:

- Data Encryption Standard (DES) for its speed and robustness. Basic messages can be encrypted using DES, with shared keys between sender and receiver.

- RSA (Rivest, Shamir, Adleman) to provide a secure method to exchange DES session keys between sender and receiver, and

- Diffie-Hellman, to provide a method of independently calculating the same key on two systems while exchanging information. Unauthorized attempts to calculate the key don't succeed using the same methods. 
- Triple-DES was used as a method of transferring private RSA keys to smaller units from larger computers. Larger computers can perform the calculations to compute the values. Using the DES algorithm multiple times with different keys on a message significantly strengthens the encryption while conserving program space on individual units [14].

\subsection{Operator Display}

The Operator Display Capabilities:

The operator display must provide a means of creating and displaying color graphic displays that will be used by the operator to monitor and control each process. Real-time values being read from the field devices should be displayed periodically in a variety of user-configurable formats.

The monitoring operator display should show the sensor network overlaid onto maps and images for better situational perspective. It is possible to monitor and manage all of the sensors from a single user interface or from more than one redundant operator stations. It should also be possible for the operator to access sensor data once he clicks on the image or name of the sensor shown on the display.

\section{Alarming:}

Each sensor can be configured to alarm at the detection high, mid, or low levels of threat. Maintenance alarms can also be configured for informational purposes.

It should be possible to display the actual contaminant concentration and the variation trend, even if surrogate parameters rather than the contaminant are measured. 
The user should be able to see whether the groundwater contamination level has reached the alarming level or not. 
CHAPTER 3 - OPTIMIZATION OF LTM SYSTEMS FOR MONITORED NATURAL ATTENUATION (MNA)

As has been discussed above, LTM systems are being used in a variety of industrial applications. In addition, the use of LTM systems for monitoring environmentally contaminated sites has been discussed with regard to sensors for indicator parameters and contaminant-specific sensors. In this chapter the example of the application and optimization of LTM systems for monitored natural attenuation will be discussed in detail.

\subsection{Monitored Natural Attenuation}

Depending on regulatory agency requirements, some form of post-closure monitoring will continue long after site cleanup has been completed (30 years is typical). Due to the regulatory requirement for extended compliance monitoring of waste units, long term monitoring will become a significant cost.

The regulatory authorities are gradually accepting monitored natural attenuation, (MNA) as a legitimate approach for remediating contaminated groundwater plumes and also for very low contaminant concentration in soils. Two conditions generally must be applied before an MNA scenario can be approved for closure of a waste unit. A source reduction technology must be applied to eliminate or drastically reduce the supply of new contaminant into the plume, and also an extensive monitoring program must be implemented to ensure that the predicted natural decay of the contaminants meets regulatory permit limits. 
A typical long-term monitoring program as required under an MNA scenario would be very expensive to operate using conventional field sampling and analysis at an off-site laboratory. Traditional long term monitoring techniques for remediation sites requires sampling on a quarterly basis or semi-annual. The subsequent laboratory analysis can take two weeks or longer. The average cost for sampling and analysis is approximately $\$ 3000$ per sample, for a comprehensive suite of constituents [15]. There is a new generation of more cost-effective in-situ monitoring technologies either commercially available or in late stages of development. Significant cost savings can be realized by installing these technologies at the remediation site and providing a means for remotely monitoring the instrument output [15].

\subsection{Issues Related to Traditional Technology}

In-situ monitoring provides faster turnaround for data interpretation giving a real time picture of the contaminant plume. Minimally intrusive monitoring technologies are required for both chemical and radionuclide contaminants. These contaminants include volatile organic compounds (TCE, PCE) as well as tritium and other radioactive contaminants. Sensors will be required to be durable such that they may continue to operate accurately without removal for as long as possible.

With remote monitoring techniques, the number of samples required from the field is drastically reduced, which also reduces the worker safety issues.

Establishment of in-situ remote monitoring technologies allows data to be collected more often and at substantial cost savings, compared to traditional monitoring techniques. This "real time" data would provide an instant picture of the plume [15]. 
For the purpose of this research, a single contaminated plume is considered. Different types of monitoring strategies are considered for this particular example. The advantages and tradeoffs for each of the strategy are discussed.

\subsubsection{Diagram of contaminated groundwater plume with monitoring wells for}

monitoring MNA process activity

The Figure 16 below shows the maximum plume boundary based on the understanding of flow, transport and attenuation processes. This example is considered for preparing comparative analyses of LTM.

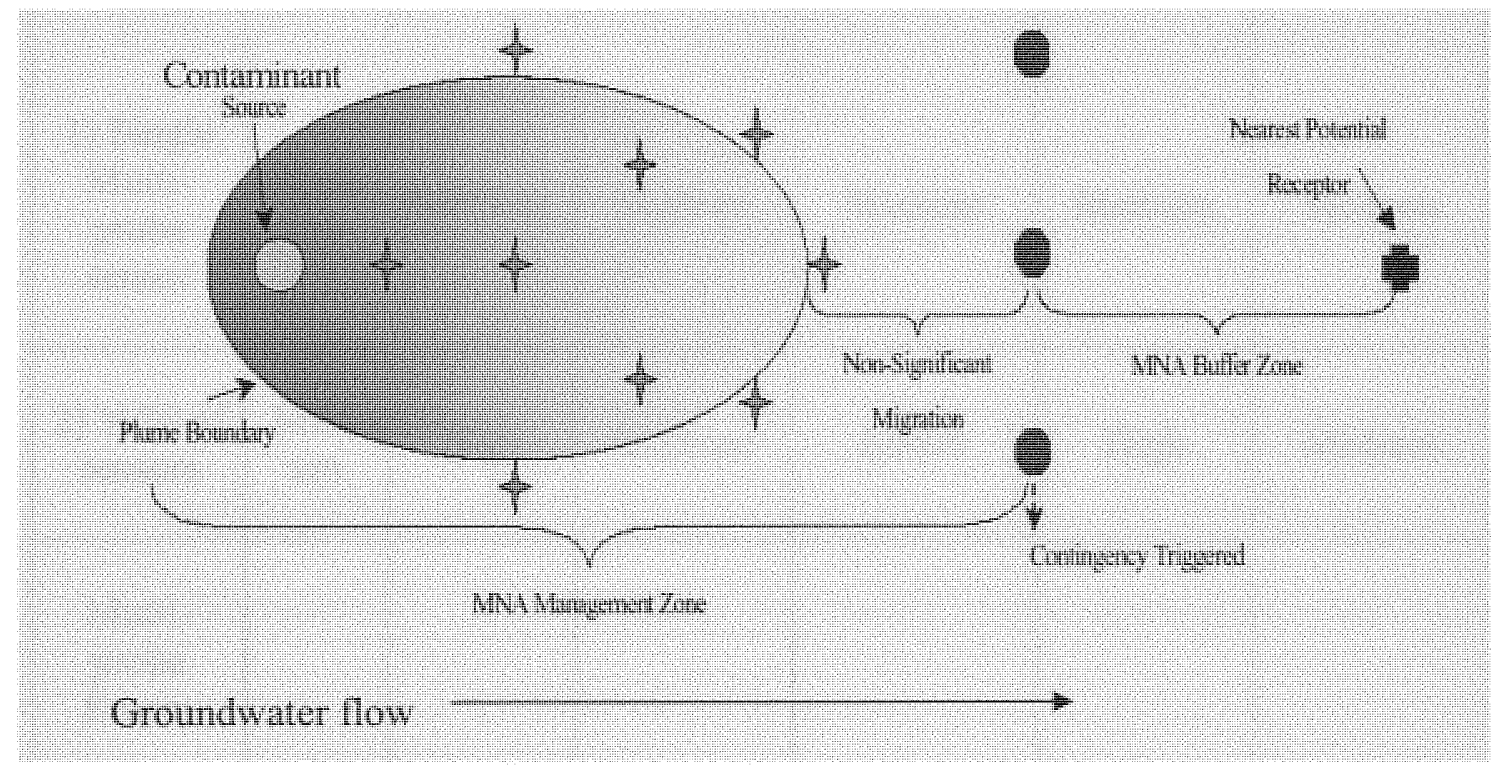

Figure 16: The Contaminated Groundwater Plume

(Source: Technical guidance for the long-term monitoring of natural attenuation remedies at department of energy sites - Office of Environmental Restoration, October 8, 1999.) 
3.3.2 LTM performance criteria

Table 3: Summary Table of LTM Performance Criteria

\begin{tabular}{|c|c|c|c|c|c|c|c|}
\hline & $\begin{array}{l}\text { LTM } \\
\text { Strategy }\end{array}$ & $\operatorname{Cost}^{I}$ & $\begin{array}{l}\text { Opera- } \\
\text { bility }^{2}\end{array}$ & $\begin{array}{l}\text { Relia- } \\
\text { bility }^{3}\end{array}$ & Durability $^{4}$ & $\begin{array}{l}\text { Mainte- } \\
\text { nance }\end{array}$ & $\begin{array}{l}\text { Data- } \\
\text { Quality }^{6}\end{array}$ \\
\hline 1 & $\begin{array}{c}\text { Well } \\
\text { Sampling } \\
\text { and } \\
\text { Analysis }\end{array}$ & High & Low & High & NA & Low & $\begin{array}{c}\text { Very High } \\
\text { (Currently, } \\
\text { the } \\
\text { Standard) }\end{array}$ \\
\hline \multirow{3}{*}{2} & $\begin{array}{c}\text { Well } \\
\text { Sampling } \\
\text { and } \\
\text { Multi- } \\
\text { Probe } \\
\text { Sensor }\end{array}$ & & & & & & \\
\hline & $\begin{array}{l}\text { 1) Single } \\
\text { Multi- } \\
\text { probe } \\
\text { sensor }\end{array}$ & $\begin{array}{c}\text { Medi } \\
\text { um }\end{array}$ & Medium & Medium & Medium & Medium & Medium \\
\hline & $\begin{array}{l}\text { 2) } N / W \text { of } \\
\text { sensors }\end{array}$ & $\begin{array}{c}\text { Medi } \\
\text { um }\end{array}$ & High & Medium & Lower & High & Medium \\
\hline 3 & $\begin{array}{l}\text { Multi- } \\
\text { probe } \\
\text { Sensor }\end{array}$ & & & & & & \\
\hline & $\begin{array}{l}\text { 1) Single } \\
\text { Multi- } \\
\text { probe } \\
\text { Sensor }\end{array}$ & Low & Medium & Low & Medium & Medium & Medium \\
\hline
\end{tabular}




\begin{tabular}{|c|c|c|c|c|c|c|c|}
\hline & $\begin{array}{l}\text { LTM } \\
\text { Strategy } \\
\end{array}$ & Cost $t^{l}$ & $\begin{array}{l}\text { Opera- } \\
\text { bility }^{2}\end{array}$ & $\begin{array}{l}\text { Relia- } \\
\text { bility }^{3}\end{array}$ & Durability $^{4}$ & $\begin{array}{l}\text { Mainte- } \\
\text { nance }\end{array}$ & $\begin{array}{l}\text { Data- } \\
\text { Quality }^{6}\end{array}$ \\
\hline & $\begin{array}{l}\text { 2) } N / W \text { of } \\
\text { sensors }\end{array}$ & Low & Higher & Medium & Low & High & Medium \\
\hline \multirow{2}{*}{4} & $\begin{array}{l}\text { Special } \\
\text { Sensors }\end{array}$ & & & & & & \\
\hline & $\begin{array}{l}\mathrm{N} / \mathrm{W} \text { of } \\
\text { sensors }\end{array}$ & $\begin{array}{l}\text { Low- } \\
\text { er }\end{array}$ & Higher & High & Low & Medium & High \\
\hline
\end{tabular}

Notes:

1. Cost - Overall cost of the implementation and operation of the strategy

2. Operability - Operability is defined here as the ease of operation of the strategy.

3. Reliability - Reliability is how the strategy is reliable in terms of making accurate contaminant concentration measurements.

4. Durability - Durability is defined as to how much time the system can be used in the field without having to be replaced, i.e. the total cost-effective life span.

5. Maintenance - How frequently the system needs inspection, repair and maintenance.

6. Data Quality - how useful is the data to find out the exact contaminant concentration, the accuracy of the data, with respect to required data quality objectives. 
3.3.3 Well Sampling and Analysis:

This is the first of all the LTM strategies that will be discussed. This is the traditional approach used for long-term monitoring. In order to study the well sampling against all the above performance parameters, the plume shown in the figure is used.

Government regulated groundwater monitoring traditionally consists of installing at least three types of monitoring wells - background, plume and down gradient - but often many more are added. Periodically each set of wells is sampled and then the samples are sent to laboratories for offsite analysis.

Well sampling and analysis is very expensive. All the samples need to be collected manually, which requires a lot of labor and in turn a lot of money. The offsite analysis, which is done at the laboratories, is expensive too.

Operability is low since this requires an operator to go in the field and collect samples. Reliability of the analysis depends on the accuracy of the sample collection and analysis of the sample in the laboratory. Manual errors can reduce the reliability of the overall result.

When a site characterization with well sampling needs to improved, the first thing one should seek to improve is the data that gets collected. Data Quality depends on the distribution of the monitoring wells over the plume. A sufficient number of wells should be installed in close proximity such that there is continuous coverage from the seasonal high water elevation down to a depth below the water table, beneath which it is unlikely that a dissolved plume will flow. The network of wells should be dense enough to provide a high degree of confidence that the plume is not migrating undetected, either between wells or beneath them. The plume should be surrounded by wells that lie outside 
the plume and samples collected from these wells should contain no trace of contamination at any depth [19] [20].

\subsubsection{Well Sampling and Multi-Probe Sensor:}

This LTM strategy involves combination of expensive well sampling and the use of multi-probe sensor. Multi-probe sensor unit measures various parameters simultaneously. These parameters are the indicator parameters like $\mathrm{pH}$, Nitrate, oxidation-reduction potential as discussed in section 2.5. These can be better indicators of early system failures. There are few commercially available multi-probe sensor units, which will be discussed later.

In this strategy, well sampling is done for some period until it is assured that the contaminant concentration is reduced below certain level. Then a multi-probe sensor is used to measure the indicator parameters and ensure that the contaminant concentration level is below the alarmed level. There should be some overlap between the well sampling and the use of multi-probe sensor in order to compare both the measurements and then build enough confidence in using only multi-probe sensor thereafter.

Using this strategy over long-term would certainly reduce the cost to some extent because the expensive well sampling and analysis will not be conducted after some period of time. Operability is low because the operator has to deploy the multi-probe sensor in the field in order to take its readings. Reliability is lower than the traditional approach because a multi-probe sensor is used instead of using well sampling and analysis for long-term. 


\subsubsection{Multi-Probe Sensor:}

Use of multi-probe sensor system to measure contaminant concentrations is the third LTM strategy being considered.

There can be only one multi-probe sensor that has to be taken in the field to take the readings, or there can be a sensor network and the data is transmitted over some communication medium.

\section{Single Multi-Probe Sensor}

In this case, the cost is lower since there is no expensive offsite analysis of samples. The probe will measure concentrations for the parameters that are available in the multi-probe sensor unit. The readings can be noted down by the operator and then taken back for analysis.

Operability is an issue since the operator has to go in the field to take the readings. Reliability is lower than the traditional approach in this case. Durability is medium, as the multi-probe sensor unit will be taken to the field only at time of taking the readings.

Data quality is not as high as the well sampling and analysis option, since the parameters being measured here are only indicator parameters and not the actual contaminant concentrations.

\section{Network of Multi-Probe Sensors}

Multi-probe sensor units can be installed in all the monitoring wells and connected to each other in a network. The data collected by each multi-probe unit will be transmitted to the central control station for the operator to monitor and analyze. 
The cost of the whole is going to increase compared to the option of using single multi-probe sensor, but it will be still very much lower than the traditional approach of well sampling. Operability is higher than single sensor as there is no need for a person to go in the field to take the readings. Reliability is also more as the data is transmitted automatically without any human help and there is no manual error. Durability of the sensors is a potential problem here and it needs to be improved if the sensors are to be installed in the groundwater for long term. Maintenance and calibration is higher than the single sensor option because one has to go in field to do that. Data quality would be medium and actual contaminant concentrations need to be interpreted from the indicator parameter measurements.

\subsubsection{Special Sensors}

There were lots of improvements in the sensor technology with a focus on ultraminiaturization through the use of microlithography during late 1990s. Many new sensors are being developed through silicon chip fabrication technologies. The sensing element is fabricated directly on the silicon chip to allow direct integration with any required signal and data processing circuitry. Not only are these sensors small, but since they can be mass-produced using standard silicon chip fabrication methods, they are expected to be inexpensive as well. These inexpensive sensors can be deployed in redundant numbers as one sensor fails; another can simply be brought online in its place without the expense of retrieval [3].

There are many special sensors available commercially as discussed in section 2.2.2, which can be installed in large numbers in the field. These are then connected to 
each other in a network and the measurements are sent to the central control station using telemetry.

Operability in this case is higher. During the lifetime of the sensor, reliability is high. The sensors are not as durable as required by the long-term monitoring of contaminated sites but if deployed in redundant numbers, there won't be much of a durability and maintenance issue. Data quality is supposed to be high.

\subsubsection{Strategies for Optimization of LTM}

Following are the two strategies that can be considered for optimization of the Long-Term monitoring system.

1) Network of multi-probe sensors i.e. indicator parameter sensors

2) Network of special sensors i.e. contaminant-specific sensors

But since sensors used in both these strategies cannot be installed in the groundwater for long periods of time, the durability of sensors is an issue. Thus significant cost savings cannot be achieved by using these strategies over the traditional well sampling approach. One approach can be used while using these two strategies is not to install the sensors directly in the groundwater. The sensor (either Multi-Probe Sensor or the Contaminant specific sensor) can be installed at one place and the groundwater samples from different monitoring wells can be pumped to that place. This way the sensor need not be placed in the groundwater for long periods of time and hence durability of the sensor can be improved substantially. 
Once it is decided to use one of the above two strategies for optimization of LTM, it is necessary to design each system, select individual optimized component from the currently available ones in the market for the system.

\subsubsection{LTM System with Network of Multi-probe Sensors:}

A. Sensors:

First it needs to be decided which indicator parameters should be measured as an alternative to measuring the actual contaminant concentration. There are three multiprobe sensors available that measure many parameters simultaneously. These sensor units are compared for type and number of the parameters measured simultaneously, accuracy and other performance characteristics. The three manufacturers are - 1) Hydrolab Inc. 2) In-Situ Inc. 3) YSI Inc. Out of these three manufacturers, following two are the best options available for the purpose and those are discussed in the following sections.

\section{Hydrolab DataSonde 4a Multi-probe sensor:}

DataSonde 4a series instruments from Hydrolab are designed for in-situ and flow through applications and it measures up to fifteen or more parameters simultaneously. It is used for remote/unattended monitoring of polluted water in both subsurface and groundwater. It comes with seven built-in expansion ports for field replaceable sensors.

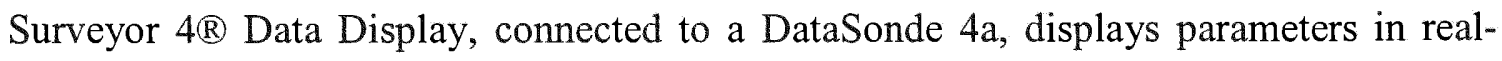
time or automatically stores or downloads more than 375000 measurements. The integral Trimble ${ }^{\circledR}$ Global Positioning System (GPS) produces location data accurate to 5 meters or less [23][24]. 
2. In-situ Troll 9000 Multi-probe sensors:

The TROLL 9000 designed with the highest quality $316 \mathrm{~L}$ stainless steel, is very rugged yet lightweight. It is capable of monitoring up to nine sensors simultaneously and is the ultimate tool for water quality profiling applications and unattended long-term deployments.

The Multi Parameter TROLL 9000 interfaces with an ultra-light, weatherized, handheld terminal or with any laptop computer or SDI-12 logger for longer-term data logging. Because of the smart sensor ports, adding sensors of choice to the specific ports can create custom sensor configurations. Also since the sensor remembers its calibration data even after being removed from the Troll 9000, it can be calibrated on a one unit and then plugged into completely different unit in the field without any need for recalibration.

It has a powerful internal data logger that allows up to 16 tests to be scheduled, run or completed and stored while a test is running. With a $4 \mathrm{MB}$ of onboard memory, a total of 1 million data points can be recorded. The low power circuitry allows Troll 9000 to be deployed for months at a time using off the shelf alkaline D-size batteries [25][26].

\section{B. Remote Terminal Units and SCADA:}

In this strategy, multi-probe sensors are installed on all the monitoring wells and Remote Terminal Units (RTU) help send the sensor data to a central monitoring station. Monitoring wells in close proximity are grouped together for the sake of data handling and data transfer and one RTU will handle this group of monitoring wells/multi-probe sensors. The figure 17 shows how the RTUs can be arranged for a particular example. 


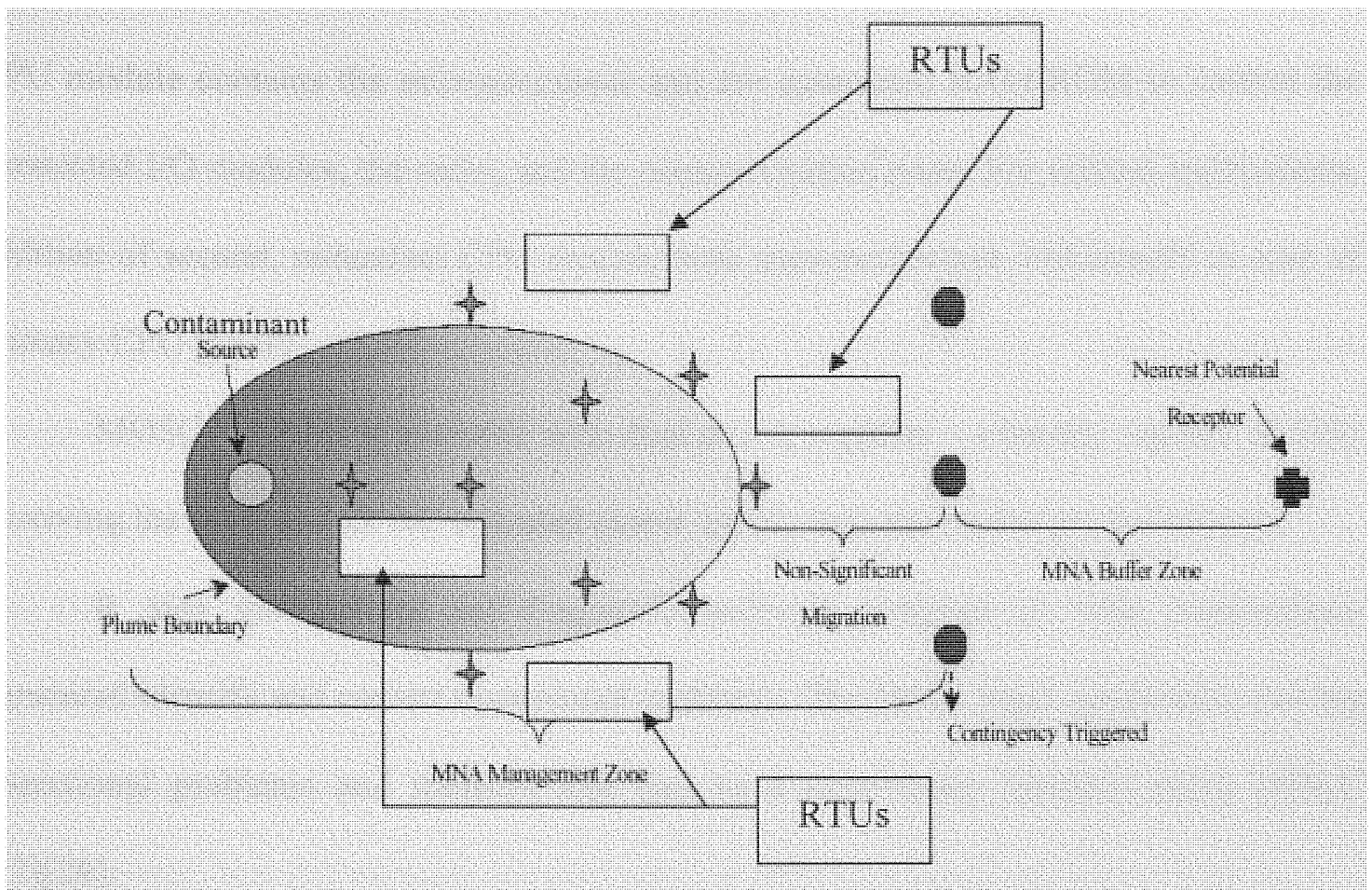

Figure 17: Possible RTU Arrangement for Typical Example (Source: Technical guidance for the long-term monitoring of natural attenuation remedies at department of energy sites - Office of Environmental Restoration, October 8, 1999.)

Following are the examples of SCADAs and a lot of RTUs that can be selected for the purpose of LTM:

\section{Motorola SCADA-MOSCAD:}

Remote Terminal Unit: MOSCAD RTU provides a data collection and processing unit. It is a microprocessor based RTU with large memory capacity that can make control decisions without the need for real-time messages from other supervisory centers (Local Intelligence). It is programmable and uses advanced symbolic ladder logic application language. Routines written in ' $\mathrm{C}$ ' may be executed as part of the total application. MOSCAD RTU uses the OSI (Open Systems Interconnection) based MDLC communication protocol for data signaling. Multiple 3 rd-party protocols are also supported. MOSCAD permits RTU-to-central and RTU-to-RTU communications. The 
CPU module contains the microprocessor and the associated RAM/ROM to control the connected $\mathrm{I} / \mathrm{O}$ modules, the radio, and the communication ports. For critical applications, addition of second, redundant CPU module with auto-switchover will benefit in case of primary CPU failure.

MOSCAD RTU is available with dual power supplies - a battery capable of fully powering the RTU and an AC operated power supply that also recharges the battery.

It also incorporates self-diagnostic software routines to help maintenance personnel identify and correct operational problems. The ladder logic application can log operational problems and transmit that data to a supervisory terminal using MDLC $[27][28]$.

I/O Modules: The 8AI Analog Input module is an accessory to the MOSCAD RTU family that allows up to $8 \mathrm{dc}$ voltages or currents. The analog voltages or currents applied to the module are multiplexed to an on-module analog-to-digital converter (ADC). The AI module also multiplexes two additional inputs to the ADC; one is logic ground to eliminate $\mathrm{ADC}$ offset-drift and the other temperature sensor to minimize the effects of ambient temperature on the accuracy of the ADC. All inputs are protected by optical isolators, which also function as the multiplex switches.

Communications via two-way radio, digital microwave radio, and wire lines is supported [27][28].

2. Campbell Scientific SCADA:

Campbell Scientific SCADA systems consist of supervisory computer running HMI (Human-Machine Interface) software and control units performing data acquisition and control functions. 
Control Units: The control units make measurements as well as report back to and execute commands from the supervisory computer. Control units function as PLCs or RTUs. They perform advanced measurement and control independent of the central computer, even if the communications to main the computer are lost. These systems are compatible with Campbell Scientific or other vendors' HMI software packages. The control units have their own UPS and during AC power loss they continue to measure and store time stamped data. Control units provide onboard statistical and mathematical capabilities. Systems are easily expandable i.e. new sensors can be added to existing sites. Control units have wide operating temperature ranges and operate in rugged environments. The control units can store up to 2 million time-stamped data points.

Sensors: Each of the control units features a variety of channel types for flexibility in measuring many different types of sensors.

Communications: Control units support multiple communication options, including radio, Ethernet, telephone, cell phone, and satellite. They also feature peer-topeer event and polled communication.

Supervisory Computer: The supervisory computer consists of a PC running either Campbell Scientific's HMI software or another vendor's software. Campbell Scientific's HMI software package like other packages provide a graphical interface that the operator uses to view the status of the remote sites, acknowledge alarms, and control the units [29].

CR10X Measurement and Control System: The CR10X consists of a measurement and control module and a detachable wiring panel. The measurement and control module measures sensors, drives direct communications and telecommunications, controls 
external devices, and stores data and programs in on-board, non-volatile storage. Typical field based CR10X systems include a data retrieval option, power supply, and environmental enclosure. To determine the best data retrieval option for any application, accessibility of the site, availability of services like cellular phone, satellite coverage, quantity of data to collect and desired time between data collection sessions needs to be considered [30].

\subsubsection{Actual Implementation of the above system:}

The above LTM strategy was tested in HCET laboratories for Department of Energy's Savannah River Project. For the test setup, multi-probe sensor DataSonde 4a from Hydrolab was used along with the CR10X data logger from Campbell Scientific. The Hydrolab's multi-probe sensor was installed at a place and the samples from different wells were pumped to that location. The multi-probe sensor unit readings were taken and all the results were well within accuracy limits except for chloride and nitrate sensors. This is the best strategy that can be used to achieve significant cost reduction over the traditional approach of well sampling.

\subsubsection{Cost Analysis:}

The cost analysis is done using RACER ${ }^{\mathrm{TM}}$ software. The Earth Tech, Inc holds all the rights for this RACER (Remedial Action Cost Engineering and Requirements) software. The detailed analysis is attached in the Appendix D.

While calculating the cost using the RACER software, some assumptions were made. It is assumed that the sensor and SCADA equipment would need to be replaced 
after every 5 yrs. It is also assumed that other equipments like pumps would need to be replaced after 15 yrs. The escalation factor is applied to the cost as well.

For the baseline sampling approach, approximate cost of collecting and analyzing one monitoring well is - (This cost includes - Sample collection, Analysis, and Labor and escalation of cost over the period of 30 years)

Total approximate cost per well for 12 months $-\$ 8500$

Total approximate cost per well for 30 yrs $-\$ 357,271$

For the multi-probe sensor approach discussed in the section 3.3.7.2, the cluster of three wells is considered and one multi-probe sensor is used for these three wells. The cost is calculated for the whole assembly and it is divided by three to get the cost per well. The approximate cost per well is as shown below -

Total approximate cost per well for 12 months - $\$ 6300$

Total approximate cost per well for $30 \mathrm{yrs}-\$ 121,319$

Thus the approximate cost per well for multi-probe sensor approach for $30 \mathrm{yrs}$ is about $1 / 3$ of the cost for the baseline sampling approach.

The figure on the next page shows the graph of the overall cost per fifteen hundred wells (Number of wells at Savannah River Site) versus the number of years.

That graph shows that approximately $\$ 350 \mathrm{M}$ of cost savings can be achieved that is about 66 percent over period of 30 years if the multi-probe sensor approach is used for fifteen hundred wells. In the graph there are two trend lines - one for the multi-probe approach and the other for the baseline sampling approach. 


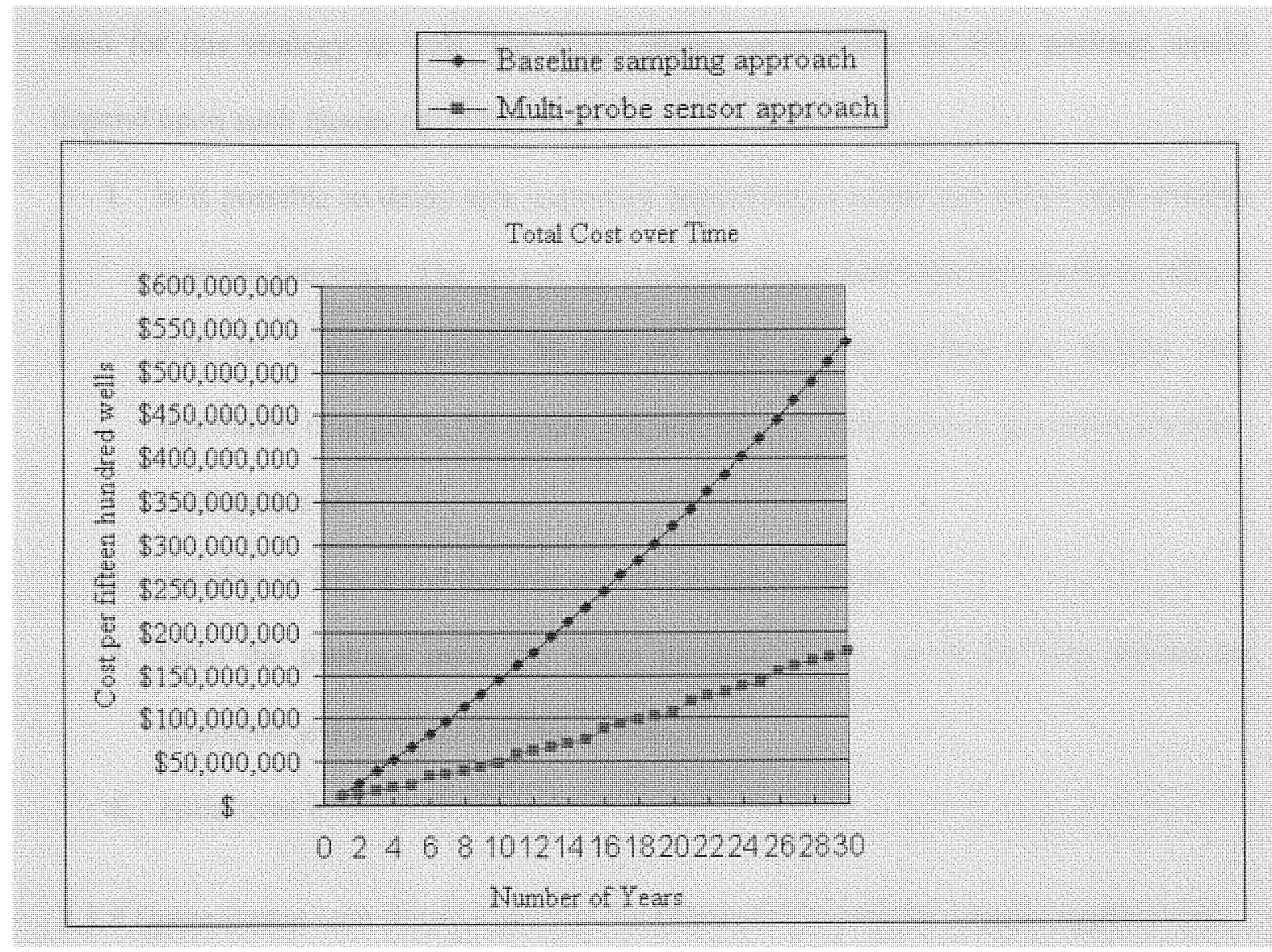

Figure 18: Graph of total cost per fifteen hundred wells versus the number of years.

\subsubsection{LTM System with Network of Special Sensors}

\section{A. Sensors:}

The contaminant specific sensors are discussed before in Section 2.2.2 and as per that, there are no sensors currently available that can be installed in the groundwater for long periods of time. The approach discussed in section 3.3.7.2 can be used for these types of sensors as well.

B. Remote Terminal Units and SCADA:

Remote Terminal Units and SCADA configuration would be same as the one used for LTM system with Network of Multi-probe Sensors. The same type of systems can be 
used for this strategy as well. The actual choice of available SCADA package would depend upon such factors as -

1. Is it possible to delay less important Input/Output scans and allow high priority scans to proceed? The reason the package should have this is because it reduces load on communications. Is this an event driven system that reports only significant changes to the central control station? This reduces the communication load quite a lot.

2. Number of Inputs and the area covered by those inputs.

3. Does the package meet the alarm handling needs, as in the way alarms are displayed and are routed to the operator?

4. Initial cost of the package and the maintenance cost

\subsubsection{Optimization with Respect to LTM Performance Criteria for specific applications:}

Two scenarios are considered where the Long-Term Monitoring system is used and then a tradeoff analysis is done for both the systems.

\subsubsection{Optimization using LTM System with Multi-Probe Sensor for a GW Plume:}

In the first scenario, the groundwater plume is moving downwards and the horizontal distance traveled by the plume is small compared to the downward movement.

For this scenario, the most appropriate monitoring system would be Long-Term Monitoring System with Multi-probe sensors. The reason behind this is that many monitoring wells are in a close proximity and one Multi-probe sensor can be used for a group of monitoring wells. 
The samples from these wells are collected to a place where the Multi-probe sensor is installed. Thus significant cost reduction is achieved, by reducing the number of Multi-probe sensors units. Multi-probe sensors units from either Hydrolab or In-situ, as discussed in the section 3.4.7.1 above, can be used.

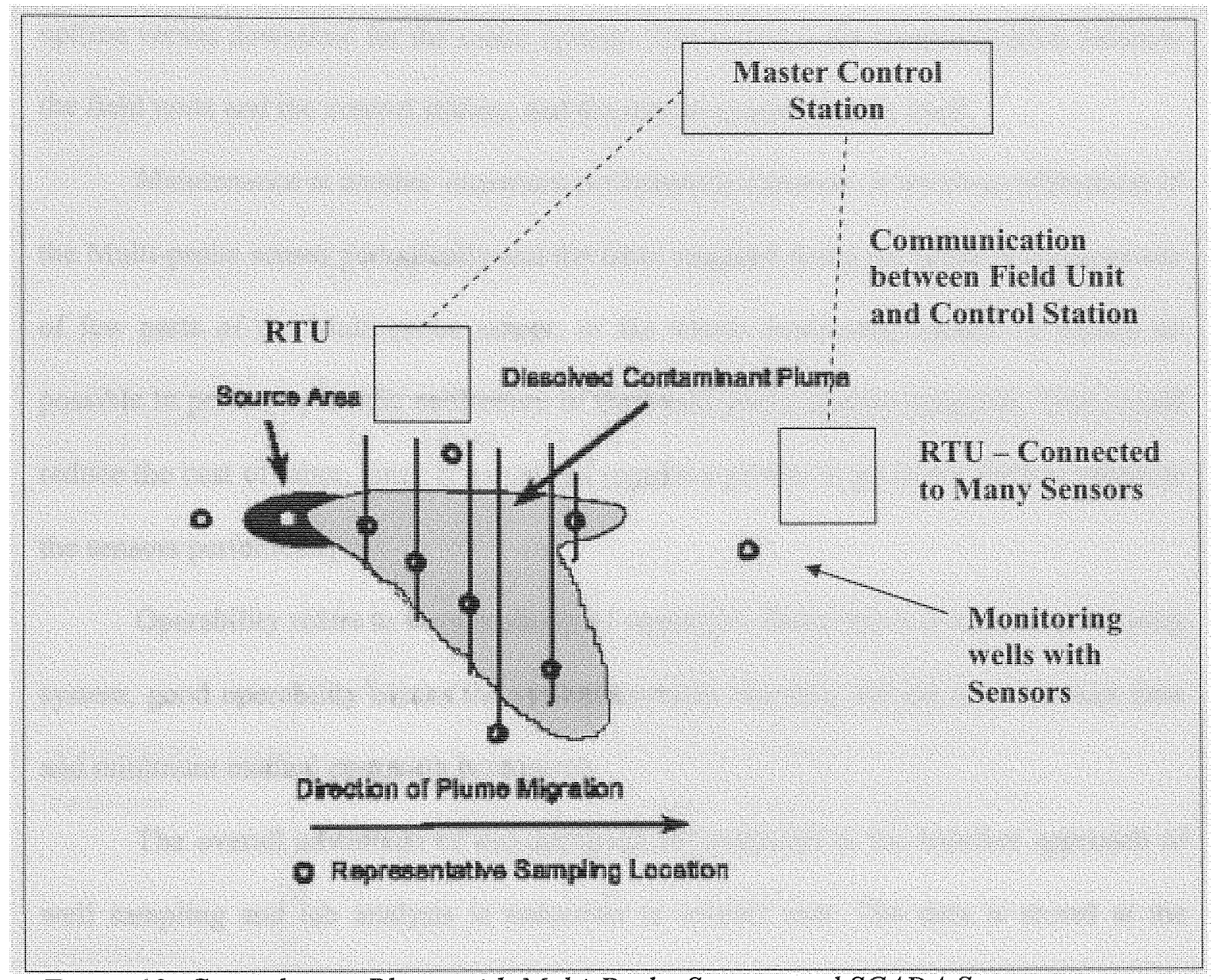

Figure 19: Groundwater Plume with Multi-Probe Sensors and SCADA System (Source: United States Environmental Protection Agency "Technical protocol for evaluating natural attenuation of chlorinated solvents in groundwater" Sept 1998)

Data quality is the most important performance criteria in this particular monitoring system configuration because the measurement in this case is indirect (i.e., measures biodegradation indicator parameters rather than the contaminant concentration itself). The indirect measurements have to be precise to a certain degree because the 
actual contaminant concentrations are inferred from these indirect measurements. The two sensors discussed offer the required accuracy and precision.

Reliability is the next most important criteria. The sensor should be reliable over a specified minimum period of time. Reliability also refers to the ratio of operational uptime versus downtime for the entire system. This includes the communication between the field units and the control station, and the master control station itself.

Maintenance is another important performance criterion. It involves calibration of the Multi-probe sensor periodically and the labor required doing so, repair or replacement of few parts of the Multi-probe sensor, or the whole monitoring system. It should be possible to perform periodic calibration of the Multi-probe sensors remotely. That would reduce the cost of labor, since it would not require a person to go in the field to calibrate the sensors periodically.

Operability refers to the simplicity of operation. Since this is a remote monitoring system, good operability means the system operates remotely for the most amount time and minimum manual operation is required.

The overall advantage of this approach as compared to the baseline approach of well sampling and lab analysis is reduction in overall cost. The data is stored at the central control station and can be retrieved at any time. Trend analysis can be done using this stored data and that can help predict future trends.

Following figures shows one of the case studies from In-Situ Inc. website. It shows the overall picture of how Troll 9000 sensors are installed in the field, how the individual multi-probe sensor is installed and how the data is transferred from the sensors to the central control station. 


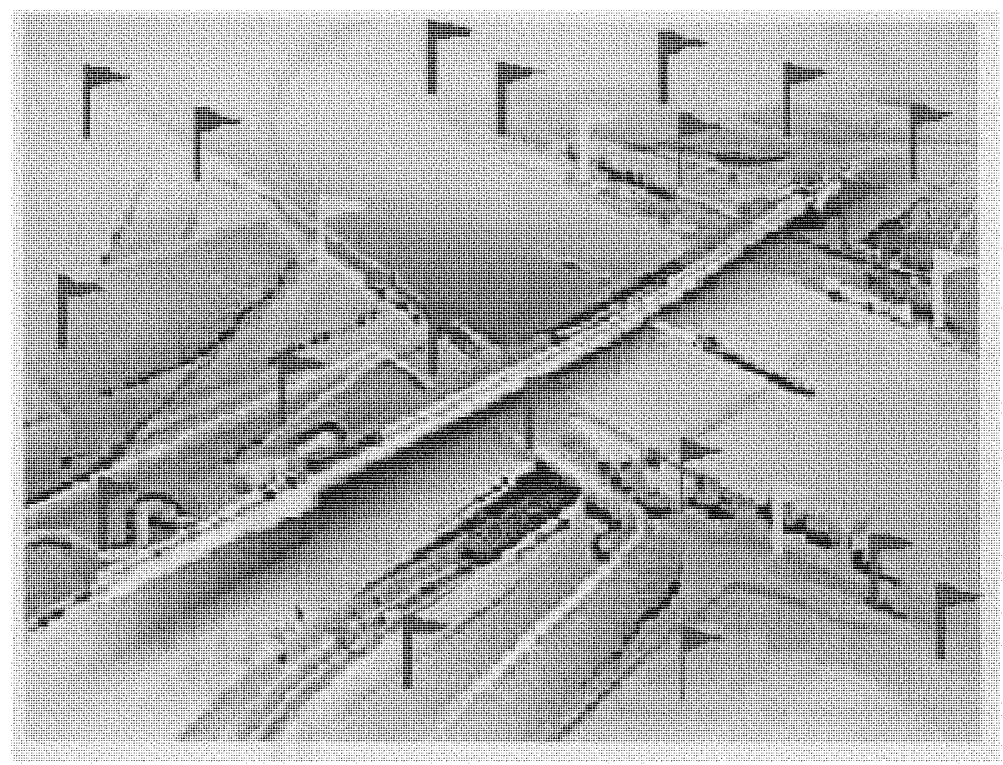

Figure 20: miniTroll sensors in the field (Source:http://www.in-situ.com/In-

Situ/Downloads/pdf/Application_Technical $\% 20$ Notes/App $\% 2012 \% 20$ Telemetry_Koend ers.pdf)

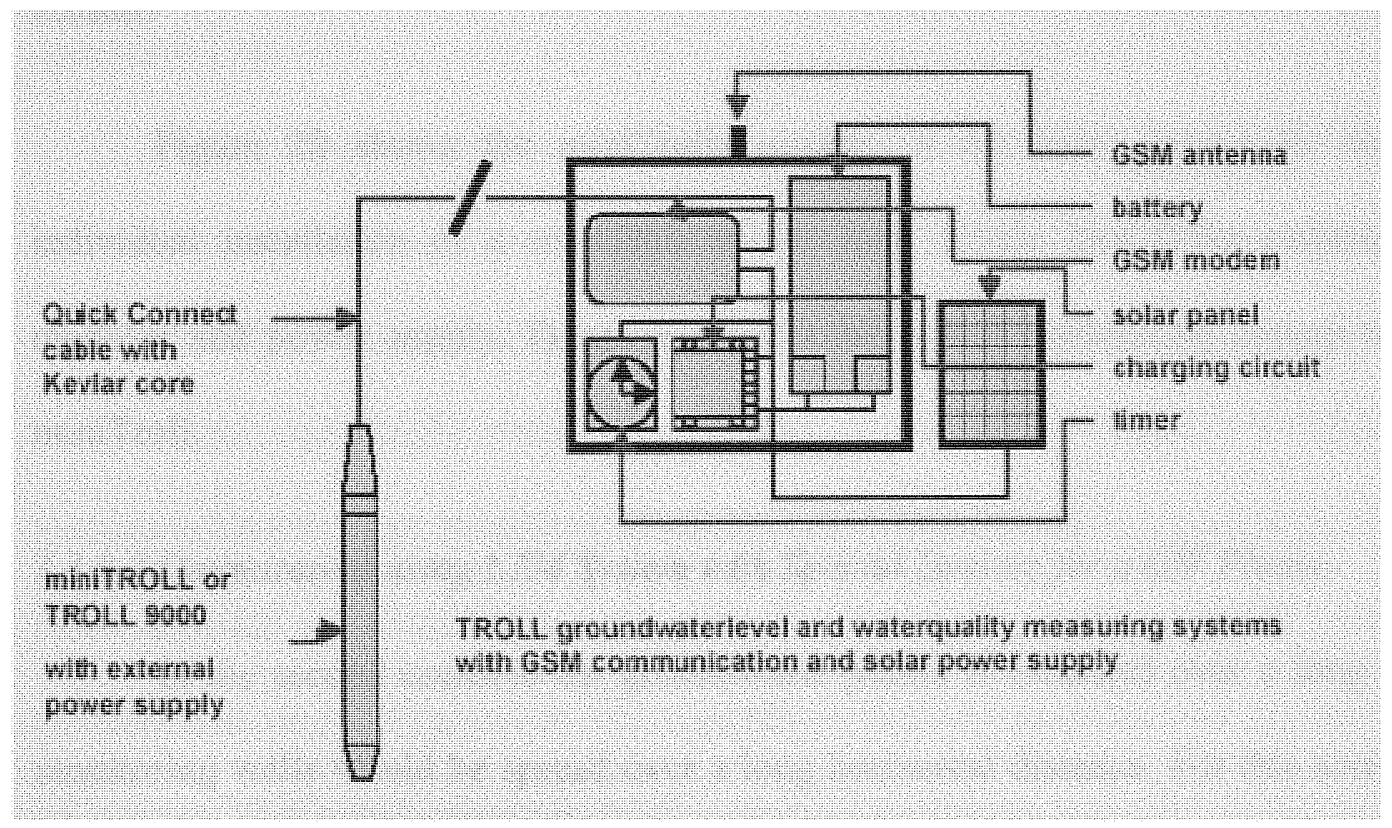

Figure 21: Individual installation of a Troll 9000 or miniTroll sensor (Source:http://www.in-situ.com/InSitu/Downloads/pdf/Application Technical\%20Notes/App $\% 2012 \% 20$ Telemetry Koend ers.pdf) 


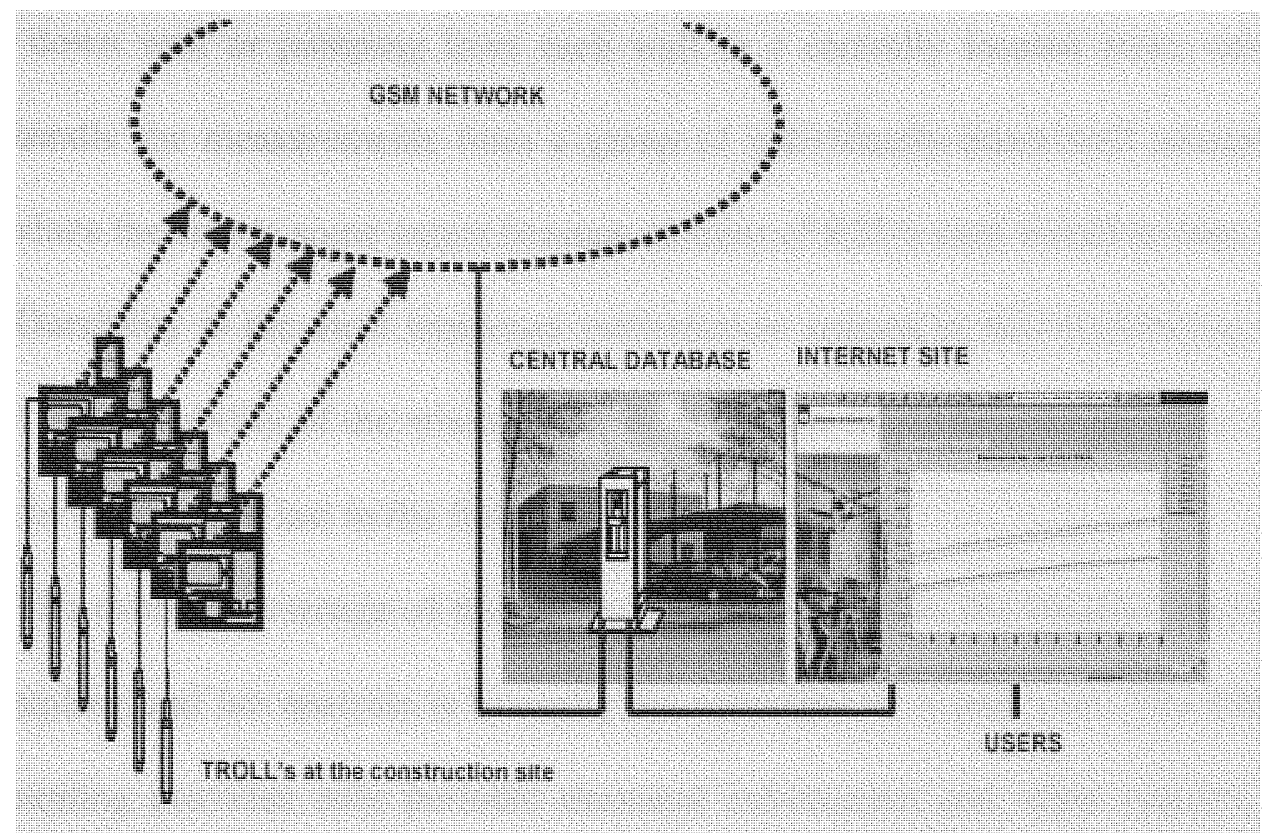

Figure 22: The Overall Monitoring System (Source:http://www.in-situ.com/InSitu/Downloads/pdf/Application_Technical $\% 20$ Notes/App $\% 2012 \% 20$ Telemetry Koend ers.pdf)

Figure 20 shows the miniTrolls sensors in the field. Figure 21 shows the individual installation of a miniTroll sensor with details like GSM communication modules and power supply. Figure 22 shows the overall monitoring system using the miniTroll.

\subsubsection{Optimization using LTM System with Special Sensors for a GW Plume:}

In the second scenario the groundwater plume is extended over a long area and monitoring wells would be spread over a long horizontal distance. For this scenario, the most appropriate Long-Term Monitoring System would be the one with the special contaminant-specific type of sensors. These sensors would be installed individually over a large area, thus preventing the need for installing individual arrays of multi-probe 
sensors down well or pumping from each well to a centrally located array; one RTU would handle the measurements from a group of individually-down-well sensors. This could reduce the cost to some extent because number of RTUs used would be less.

Durability is the most important performance criteria in this monitoring system strategy. The reliability of the whole system depends on how much longer these sensors are able to operate and last in the field.

The overall advantage of this approach as compared to the baseline approach of well sampling and lab analysis is basically the reduction in overall cost. The traditional well sampling provides only periodic chemical information and often requires highly trained operators using sophisticated and expensive equipment. But this approach offers real-time continuous information using a relatively inexpensive system with automatic operation. [31].

Following paragraph describes a particular case study in which portable acoustic wave sensor system is used for in-situ field screening of volatile organic compounds.

Portable acoustic wave sensor (PAWS) systems have been developed for realtime, online, and in-situ monitoring of volatile organic compounds (VOCs). These systems utilize the high sensitivity of surface acoustic wave devices to changes in mass or other physical properties of a film cast onto the device surface. PAWS systems are portable and easy to implement. Demonstrations indicate sensitive and accurate analysis of isolated VOCs and agreement with baseline instruments typically within 10 percent. Minimum detection levels for typical VOCs are about 1 to $10 \mathrm{ppm}$. The calibrations are stable; for example, in the last field demonstration, calibrations before and after the test were within 1.5 percent, even following the large amount of handling experienced during 
the field demonstration. The systems are designed to enable online baseline analysis so they can be used continuously for long-term monitoring. Response is rapid and reversible, so concentration fluctuations can be monitored in real time on a few-second time scale. The systems can be configured for either aboveground monitoring or in situ analysis in vadose zone monitoring wells [31].

Calibration of the sensor device is performed by challenging it with known concentrations of the chemical of interest. The SAW devices are sensitive to the mass of chemical absorbed on the device surface; they will detect adsorption of water vapor. Periodically ambient air should be directed to the sensor to purge the sensor of the contaminant and to reestablish sensor baseline. System stability dictates how often sensor baseline must be reevaluated [31].

The monitoring using this sensor can be implemented in one of the two ways:

1. Packaging the sensor, electronics, and gas handling hardware and inserting them into the monitoring well at a point above the groundwater for in-situ analysis. Or

2. Extracting a gas sample from the well through a long tube and delivering it to an aboveground sensor package [31].

\subsection{Conclusions and Recommendations}

In earlier chapters different available LTM components were discussed and the concept of LTM was developed. In this chapter optimization of LTM for monitored natural attenuation was discussed in detail. First, different monitoring strategies - well sampling \& analysis, well sampling \& multi-probe sensors, LTM with multi-probe sensors and LTM with special (contaminant specific) sensors - were discussed. Then out 
of these strategies two were selected for optimization of LTM. Each strategy discusses which sensors and monitoring systems can be selected for optimization. It was also discussed how optimization is achieved with respect to the LTM performance criteria.

Then two specific examples - one is LTM system with multi-probe sensor for a GW Plume and the other is LTM system with special sensor for a GW Plume - are discussed. It is also discussed how optimization of LTM is achieved in these two examples. Along with this two case studies are discussed.

In the design and optimization of long-term monitoring systems for contaminated environmental sites, the use of either multi-probe or contaminant specific - special sensor systems, including data acquisition system / SCADA and its components, and the approach as discussed, have been shown to offer significant performance advantages over the baseline monitoring approach of well sampling and lab analysis. Long-term monitoring systems with multi-probe sensors would be the most appropriate and optimized monitoring system for vertical groundwater plume. Vertical groundwater plume is where the plume travels vertically more than it travels horizontally. Long-term monitoring system with special (contaminant specific) sensors can be the most appropriate and optimized system for a groundwater plume that travels over larger horizontal distance. However, the specific monitoring approach, equipment, and implementation will depend largely on the specific environmental site and monitoring objectives. Many commercially available options exist for sensors, SCADAs.

The cost analysis of the baseline approach and the multi-probe sensor approach shows that significant cost reduction can be achieved by using the multi-probe approach over the baseline approach. With the multi-probe sensor approach and for a period of 30 
years, for one well about $\$ 238 \mathrm{~K}$ of cost savings can be achieved and for a particular application of Savannah River site with approximately fifteen hundred wells about $\$ 350 \mathrm{M}$ of cost savings can be achieved, which is about 66 percent of cost savings.

Recommendations for further studies and developments might include (1) developing concepts for networking sensor arrays, (2) field testing actual monitoring strategies to evaluate their feasibility under actual field conditions, e.g., limitations on telemetry due to site topography, (3) field testing of actual monitoring systems to evaluate actual data quality achieved and maintenance issues under longer term operating conditions. 
[1] Hanadi S. Rifai, Philip B. Bedient, and Gretchen L. Shorr. "Monitoring hazardous waste sites: characterization and remediation considerations" Journal of Environmental Monitoring, 2, 2000, 199-212

[2] Applied Research Associates. "Long-Term Monitoring Sensor Network" http://www.netl.doe.gov/products/em/IndUnivProg/pdf/3161.pdf , Office of Science and Technology TMS Tech ID: 3161, Sept 2002, 63-64

[3] Brian B. Looney, Ronald W. Faltra. "Vadose Zone Science and Technology Solutions - Volume 1", Battelle Press, 510-563

[4] Cullen S.J., J.H. Kramer, and L.R. Luellen. "A Systematic Approach to Designing a Multiphase Unsaturated Zone Monitoring Network," Ground Water Monitoring and Remediation, 15(3), (1995): 124-135.

[5] Cullen. S.J., "Vadose Zone Monitoring: Experiences and Trends in the United States," Ground Water Monitoring and Remediation, 15(3) (1995): 136-144.

[6] Choudhury, P.K., "Optical Sensors in Environmental Monitoring," Current Science, 74(9) (1998): 723-725.

[7] Chang S.M., Y.H. Kim, J.M. Kim,Y.K. Chang, and J.D. Kim. "Development of Environmental Monitoring Sensor Using Quartz-Crystal Micro-Balance," Molecular Crystals and Liquid Crystals Science and Technology Section A-Molecular Crystals and Liquid Crystals, 267 (1995): 405-410

[8] Rodgers, K.R., and E.J. Poziomek. "Fiber Optic Sensors for Environmental Monitoring," Chemosphere, 33(6) (1996): 1151-1174.

[9] Kounaves, S.P., W. Deng, P.R. Hallock, G.T.A. Kovacs, and C.W. Storment. "Iridium-Based Ultramicroelectrode Array Fabricated by Microlithography," Analytical Chemistry v 66(3) (1994): 418-423

[10] Department Of Energy, USA. "Long-Term Monitoring Sensor and Analytical Methods Workshop Report", Workshop in Orlando, FL. June 13-15, 2001

[11] Hydrolab - Hach Company, Technical Support, Technical Notes. Link to the webpage: http://www.hydrolab.com/TechSupport.html

[12] Carey, Kevin (Microwave Data Systems). "Planning improves reliability in radiobased SCADA systems", Pipe line \& gas industry, volume 82, February 1999, 41-45.

[13] Jones, Jeremy. "How do you get successful SCADA?", Control \& Instrumentation Journal, 24(10), October 1992, 34-36. 
[14] John A. Kinast, Manager, Systems development, Energy systems and business analysis, Institute of gas technology, William F. Rush, Jr., Ph.D., Assistant institute physicist, Gas operations technology, Institute of gas technology. "Encryption provides low-cost SCADA operating security". Link to the webpage: http://www.bandwidthmarket.com/resources/speeches/ops/kinast/entelec-scadasecurity.doc

[15] General reference information. "Long term monitoring technologies need/opportunities", Need/Opportunity ID No.: SR02-3029, October 2001. Link to the webpage: http://www.srs.gov/general/scitech/stcg/Needs/02-3029.htm

[16] Donna L. Hale, P.E, Michael S. Roddy, P.G, and Timothy R. Smail. "Deployment Issues for sensors for long-term monitoring applications", International containment and remediation technology conference, June 2001. Link to the webpage: http://www.containment.fsu.edu/cd/content/pdf/419.pdf

[17] Onset Computer Corporation. "What is a data logger", Link to the webpage: http://www.onsetcomp.com/MiscInfo/3564 whatlogger.html

[18] "Spread Spectrum Scene- SCADA Stuff", Link to the webpage: http://www.SsSmagc.com/scada.html

[19] Hul White. "Do Monitoring Wells Monitor Well? Part I", LUSTLine Bulletin 40, Link to the webpage: http://www.epa.gov/swerust1/cat/LL40WanderLust.pdf

[20] Hul White. "Do Monitoring Wells Monitor Well? Part II", LUSTLine Bulletin 41, June 2002. Link to the webpage: http://www.epa.gov/swerust1/cat/LL41WanderLust.pdf

[21] Campbell Scientific, Inc., SCADA Applications. Application in Water Quality Monitoring - Water Conservancy District. Link to the webpage: http://www.campbellsci.com/centers/scada-zjvwcd.html

[22] Bentek Systems, SCADA \& Telemetry Solutions. "SCADA Applications - water, Municipal Water System - tail end data extension \& wide area SCADA". Link to the webpage: http://www.scadalink.com/slappwatdataextend5.htm

[23] Hydrolab Inc. Link to the webpage: http://www.hydrolab.com/Products.html

[24] DataSonde $® 4$ and MiniSonde ${ }^{\circledR}$ Water Quality Multiprobes User's Manual. Link to the webpage: http://www.hydrolab.com/PDFs/Series\%204A\%20Manual.pdf

[25] In-Situ Environmental Monitoring Instrumentation and Systems. Link to the webpage: http://www.in-situ.com/In-Situ/Products/Products.html

[26] In-Situ Inc. Product specification sheets. Link to the webpage: http://www.insitu.com/In-Situ/Downloads/Downloads_SpecSheets.html 
[27] MOSCAD - Motorola SCADA Documentation. Link to the webpage: http://www.moscad.com/documentation.html

[28] MOSCAD - Motorola SCADA Remote Terminal Unit features. Link to the webpage: http://www.itsinfo.com/moscadpdf/MOSCAD.PDF

[29] Campbell Scientific Inc. SCADA Info Center. Link to the webpage: http://www.campbellsci.com/centers/scada.html

[30] Campbell Scientific Inc. SCADA - Control Units Documentation - CR10X Measurement and Control System Specifications. Link to the webpage: ftp://ftp.campbellsci.com/pub/outgoing/lit/b cr10x.pdf

[31] Gregory C. Frye-Mason, Sandia National Laboratories, Albuquerque, New Mexico "In situ field screening of volatile organic compounds using a portable acoustic wave sensor system"

[32] The Interstate Technology and Regulatory Cooperation Work Group, In Situ Bioremediation Work Team "Natural Attenuation of Chlorinated Solvents in Groundwater: Principles and Practices" - Final - May 1999.

[33] Clifford K. Ho, Michael T. Itamura, Michael Kelley, and Robert C. Hughes, "Review of Chemical Sensors for In-Situ Monitoring of Volatile Contaminants", Sandia Report (SAND2001-0643) March 2001

[34] H. Gvirtzman, M.J. Pinto, and S.M. Gorelick, "In-Well VOC Removal", Link to the webpage: http://pangea.stanford.edu/hydro/research/in-well/in-well content.htm

[35] Troll's guarding construction site and performing at Internet. Applications of Troll 9000. Link to the webpage: http://www.in-situ.com/InSitu/Downloads/pdf/Application_Technical\%20Notes/App $\% 2012 \% 20$ Telemetry_Koend ers.pdf 
Appendices 


\section{Appendix A: Indicator Parameters}

Following are some of the Indicator Parameters and usefulness of their measurement. Ammonium

Ammonium, $\mathrm{NH} \mathrm{4+,} \mathrm{is} \mathrm{an} \mathrm{ionized} \mathrm{form} \mathrm{of} \mathrm{nitrogen.} \mathrm{Nitrogen} \mathrm{compounds}$ dissolved in water are usually classified as organic or inorganic. Soluble inorganic nitrogen compounds include ammonia, nitrite, and nitrate. Nitrate is related to ammonia in that nitrifying bacteria convert ammonia to nitrate, which is less toxic to animal life. Ammonia has two forms - the ammonium ion, and unionized, dissolved ammonia gas (NH 3). The form depends on $\mathrm{pH}$, with ammonium predominating when the $\mathrm{pH}$ is below 8.75 , and ammonia predominating above $\mathrm{pH} 9.75$. The forms are freely interconverted during a change in $\mathrm{pH}$. Total ammonia is the sum of ammonium and ammonia concentrations [11].

Usefulness of Ammonium Measurement

Biologically available nitrogen is found in both suspended solids and dissolved compounds in natural waters. Many natural waters are nitrogen-limited, meaning that nitrogen compounds are the limiting nutrients. Thus even small changes in biologically available nitrogen levels can dramatically effect the levels of microbiological, plant, and eventually, animal life. High levels of accessible nitrogen, of which total ammonia is one form, can lead to an over abundance of microorganisms, a situation which often results in mortality to higher organisms (such as fish and shrimp) because of depleted dissolved oxygen. Excessive total ammonia can also result in mortality to the higher organisms, especially when high $\mathrm{pH}$ levels favor dissolved ammonia gas, which is more toxic than 
the ammonium form. Applications of ammonium ion measurement include tracing the movement of point or non-point source pollutants [11].

\section{Chloride:}

Chloride is an ionized form of chlorine. Chloride can indicate intrusion of seawater or other saline waters into underground drinking water supplies. Chloride is often used as an "early warning" tracer for leakage of contaminants into the ground water since it is highly mobile in the earth, where diffusion of many organic compounds is slowed down by their interaction with rocks and soil [11].

The usefulness of chloride measurement includes the following:

- The chloride ion does not react with, or adsorb to, most components of rocks and soils, and so is easily transported through water columns. Thus chloride is an effective tracer for pollution from chemicals moving from man-made sources into natural water bodies, or for salt-water intrusion.

- Applications of chloride ion measurement include monitoring landfills for leaks, tracing the movement of point-or non-point source pollutants within a natural water body, detection of salt-water intrusion into drinking water supplies (ground or surface waters) [11].

\section{Conductivity:}

Conductivity is a measure of the ability of water to pass an electrical current, which increases with the amount of dissolved ionic substances that the water contains. Conductivity is inversely related to the resistance of a solution, and is measured in units of mhos $/ \mathrm{cm}$. The conductivity of water increases dramatically with increasing 
temperature; the exact amount is determined by the individual ions present in the solution. Specific conductance is the most common unit used, where the conductivity has been corrected to 25 degrees $\mathrm{C}$. The probe measures the current between 2 electrodes held at a fixed potential; additional electrodes are used to compensate for any fouling of the electrode surfaces [11].

Depth

Depth is the vertical distance between the probe and the surface of the water. A pressure transducer is used to measure the pressure at a particular point in the water column, and depth is calculated using the average density of water while compensating for any salt content. This allows the user to construct vertical profiles of other parameters in a water column [11].

Dissolved Oxygen (D.O.)

Dissolved oxygen is the measure of the amount of oxygen present in water and available for respiration. D.O. is measured in milligrams per liter as well as in percent saturation. Oxygen is less soluble in warm and salty waters, so the instrument compensates the apparent percent saturation for changes in temperature and conductivity. The probe (Clark cell) measures the current resulting from the electrochemical reduction of oxygen (at a gold cathode) diffusing through a selective membrane. Sufficient sample flow is required to prevent falsely low readings due to depletion of oxygen in the solution in front of the probe [11]. 
Nitrate:

Nitrate is an ionized form of nitrogen. Nitrogen compounds dissolved in water are usually classified as organic or inorganic. Soluble inorganic nitrogen compounds include ammonia, nitrite, and nitrate. Nitrifying bacteria convert ammonia to nitrate, which is less toxic to animal life [11].

The usefulness of Nitrate measurement includes the following:

- Nitrogen is an essential nutrient for all forms of life, including all levels of aquatic organisms. Biologically available nitrogen is found in both suspended solids and dissolved compounds in natural waters.

- Many natural waters are nitrogen-limited, meaning that nitrogen compounds are the limiting nutrients. Thus even small changes in biologically available nitrogen levels can dramatically effect the levels of microbiological, plant, and eventually, animal life. High levels of accessible nitrogen, of which nitrate is one form, can lead to an over abundance of microorganisms, a situation which often results in mortality to higher organisms (such as fish and shrimp) because of depleted dissolved oxygen [11].

Turbidity:

Turbidity is a measure of the clarity of a liquid, measured in NTUs (Nephelometric Turbidity Units). Turbidity particles cause light to be scattered and absorbed rather than transmitted in straight lines through a sample. Turbidity is the opposite of clarity. Turbidity probes measure the intensity of infrared light scattered at 90 degrees from the incident beam [11]. 


\section{Temperature}

Temperature is a measure of heat present in water. Aside from dissolved oxygen, temperature is considered the single most important parameter. Knowledge of water temperature is essential to the measurement of dissolved oxygen, conductivity (salinity), $\mathrm{pH}$, and virtually every other water quality parameter [11]. Biological processes typically are influenced by environmental temperature. e.g. the rate at which microbial enzymes degrade organic contaminant.

\section{Redox / Oxidation-Reduction Potential (ORP) / (Eh)}

Redox / ORP are terms for the voltage measured at an inert electrode, reflecting the extent of oxidation of a water sample. The more positive the ORP of a solution, the more oxidized are the chemical components of the water (less positive indicates less oxidized, or more reduced). The probe consists of a platinum electrode, whose potential is measured with respect to a reference electrode; this electrode rapidly comes into equilibrium with the potential of the sample solution. Because of the complex relationship between ORP and temperature, no compensation is attempted; it is thus best to report both the ORP and temperature of a water sample [11]. Anaerobic microbial degradation of organic contaminants usually takes place at very low oxidation-reduction potential, e.g. $-150-400 \mathrm{mV}$

\section{Salinity}

Salinity is the measure of the total quantity of dissolved salts in water. Salinity is measured in parts per thousand (ppt) or practical salinity units (psu). Salinity is calculated 
from the conductivity of the solution, and is usually the preferred unit when studying bay, estuary and ocean waters [11].

\section{Appendix B: Sensors}

Multi-probe Sensors:

DataSonde 4a, MiniSonde 4a Multiprobes and data displays by Hydrolab

The DataSonde Series 4a water quality instruments are designed for use in fresh, salt or polluted water. It is designed for in-situ and flow through applications and measure upto 15 or more parameters. It comes with 7 built in expansion ports for field replaceable sensors.

It can measure following parameters:

- Temperature

- $\mathrm{PH}$

- ORP

- Conductivity

- Dissolved oxygen

- Depth

- Turbidity

- Total dissolved gas

- Ammonia

- Nitrate

- Chloride

- Ambient light

- Transmissivity

- Chlorophyll 
MiniSonde $4 \mathrm{a}$ has four built-in ports and new sensors can be added as needed. Data can be downloaded to the Surveyor $4 \mathrm{a}$ or directly to a PC.

The Surveyor 4a provides data logging and display. It is connected to the DataSonde 4a or MiniSonde $4 \mathrm{a}$ and displays water quality parameters in real time or automatically stores.

Troll 9000 by In-Situ - Main Features:

\section{Smart Sensors:}

The sensors are plug and play type. They can be plugged in any sensor port, which allows for custom and redundant sensor configuration. The sensors can remember their calibration coefficients, so even if they are calibrated on one unit and are plugged into completely different unit, there is no need for recalibration.

\section{Digital D.O.:}

With this technology we can choose digital sleep mode for long term monitoring where no stirring required or it can measure D.O. in Continuous ON mode for extreme accuracy. Both will give the highest level of accuracy.

In general stirring greatly improves readings from ISE sensors such $\mathrm{pH}, \mathrm{ORP}$, nitrate, Chloride, ammonium etc. Up to this time, stirring with field instruments has not been practical due to the excessive power requirements. Additionally, stirrers that are currently available on some multi-parameter units are simply too turbid on the sample to obtain quality measurements.

In-situ's stirrer solves these problems by slowly stirring the sample and it is powered by D-size alkaline battery that lasts for 6 months. 


\section{Pre-calibrated:}

All units are pre-calibrated and are ready to use right out of the box. Each unit is subjected to intense environmental testing. Calibration is certified to NIST traceable standards. A calibration report is included with every unit. The sensors are automatically compensated real-time.

\section{Single "Quick-Cal" Solution:}

The TROLL 9000 is placed in the solution and just by pressing CALIBRATE, the sensors can be calibrated. Quick-Cal solutions can be used to simultaneously calibrate D.O., conductivity, $\mathrm{pH}$ and ORP sensors.

\section{Most Sensors in a 1.75" O.D.}

Troll 9000 features the most sensors in a 1.75 " housing.

\section{Powerful Datalogger:}

Internal logger allows up to 16 tests to be scheduled run or completed and stored while a test is running. With $4 \mathrm{MB}$ of onboard memory, over 1-million data points can be recorded. Since memory is non-volatile, memory is always protected even if power is disconnected. Also it has a low power circuitry so that it can be deployed for months at a time using alkaline D-Size batteries. It comes with surge and lightening protection.

\section{Software:}

Pocket-Situ is used for Surveying and profiling. It can be used for viewing and downloading data in real time. It is used to start and stop logging operations, manage schedule, save contacts. It comes with a watertight case.

Win-Situ is easy to use software. It has a wizard-based interface that is used from calibration to test setup. Data can be viewed real time as a graph or report. Some of the 
features of the software include - There is a tree organization of instruments, parameters and tests and by selecting a device in the tree it displays its parameters and tests. It has Wizards that helps in connecting new devices, setting up tests, calibrating sensors and editing parameters. It has icons for Scheduled tests, Running tests, Manual-start tests and completed tests for a device. It has readouts that display remaining memory and battery voltage.

\section{Other Features:}

It is designed with $316 \mathrm{~L}$ stainless steel and it's rugged yet lightweight. MP Troll 9000 is offered in six different versions. Versions are scaled to meet the needs and budgets of the organizations and individuals needing it. 


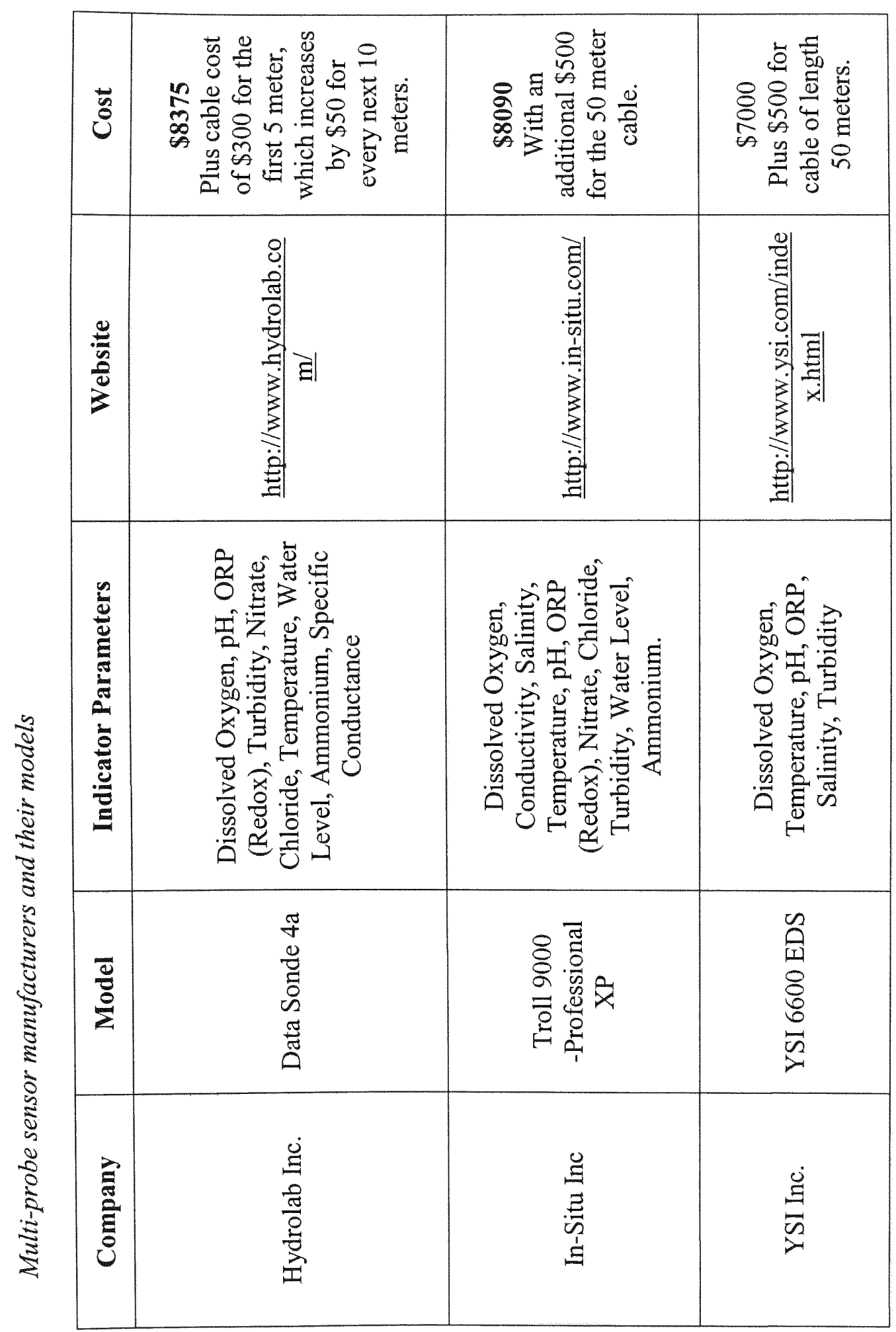




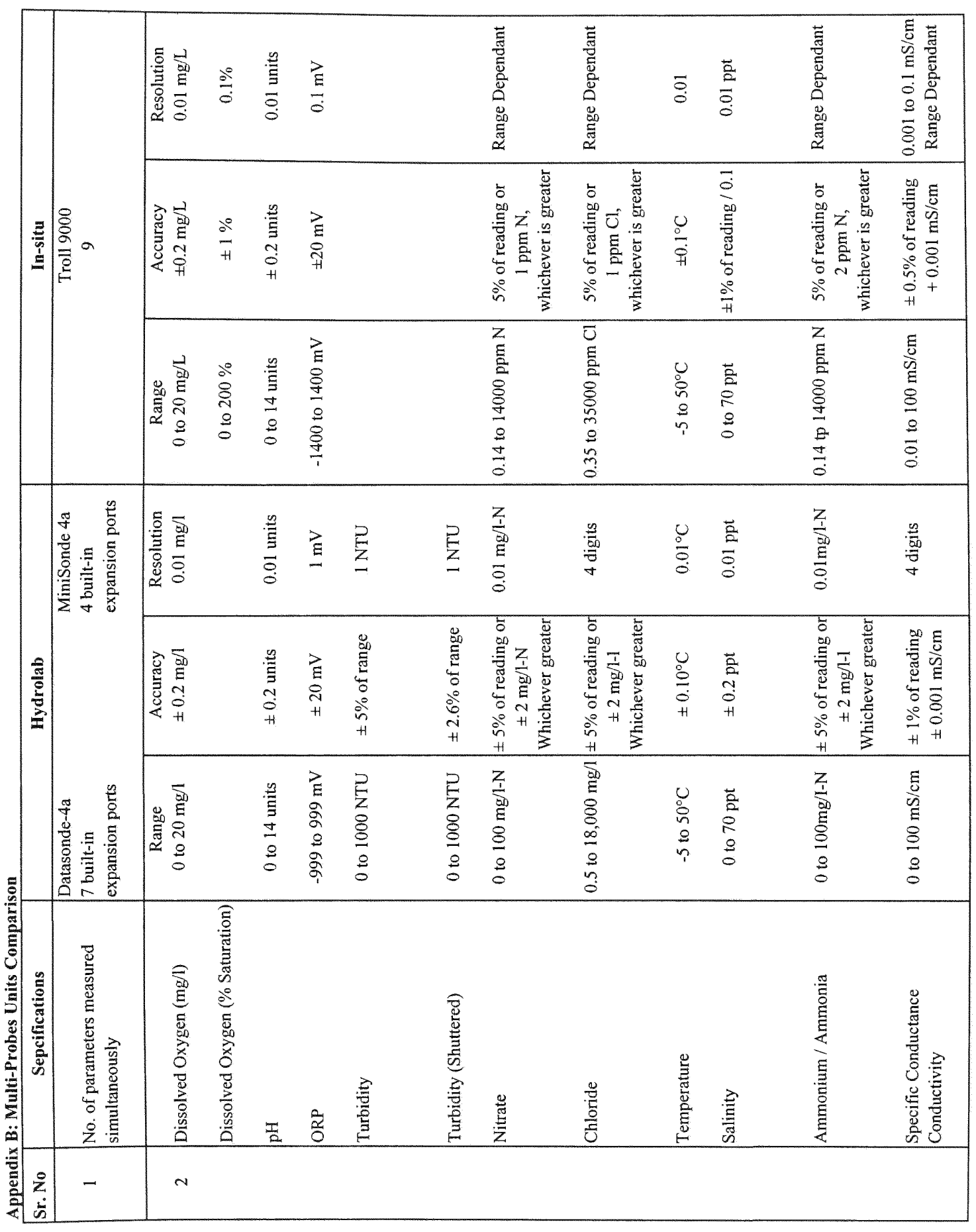

2 


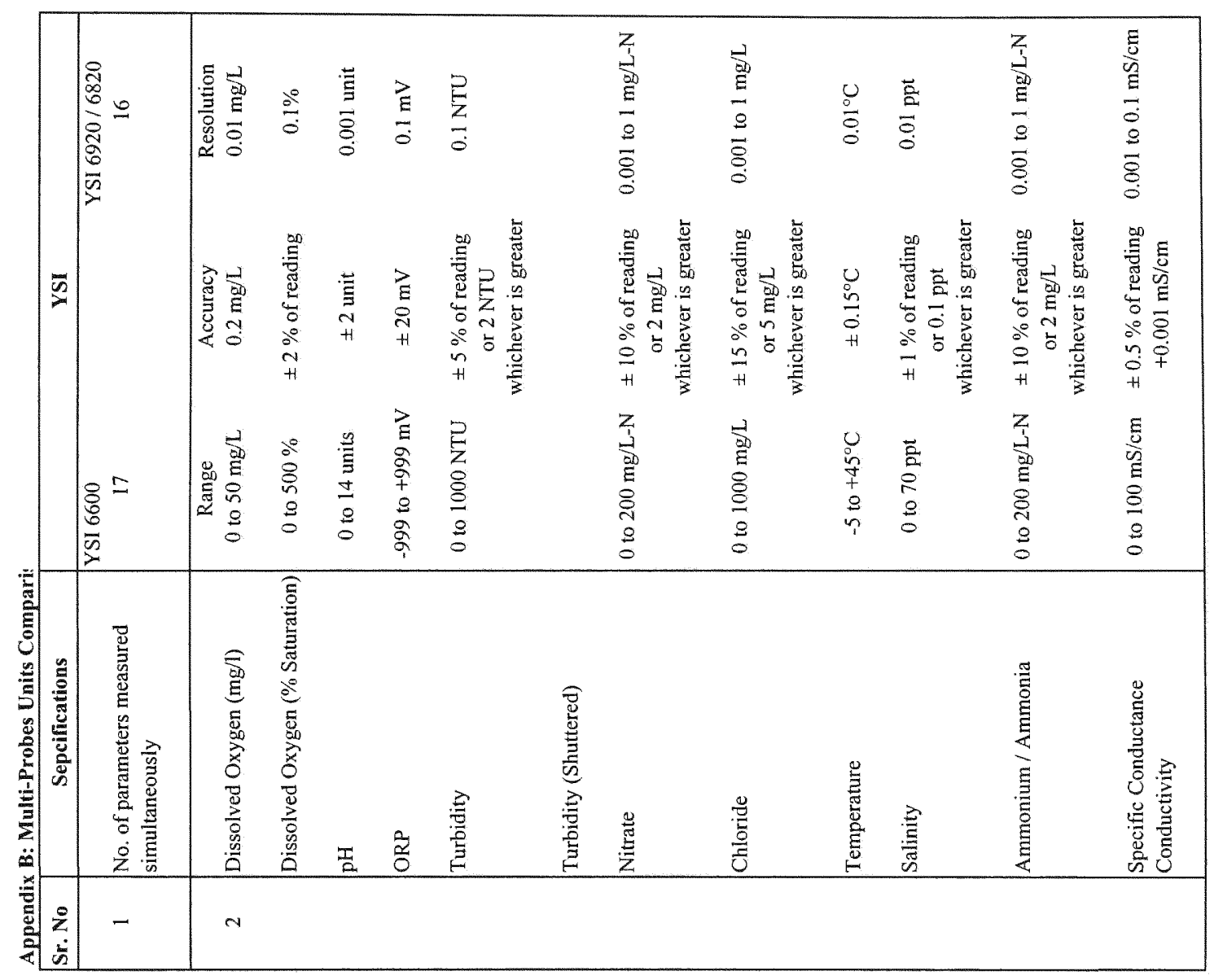




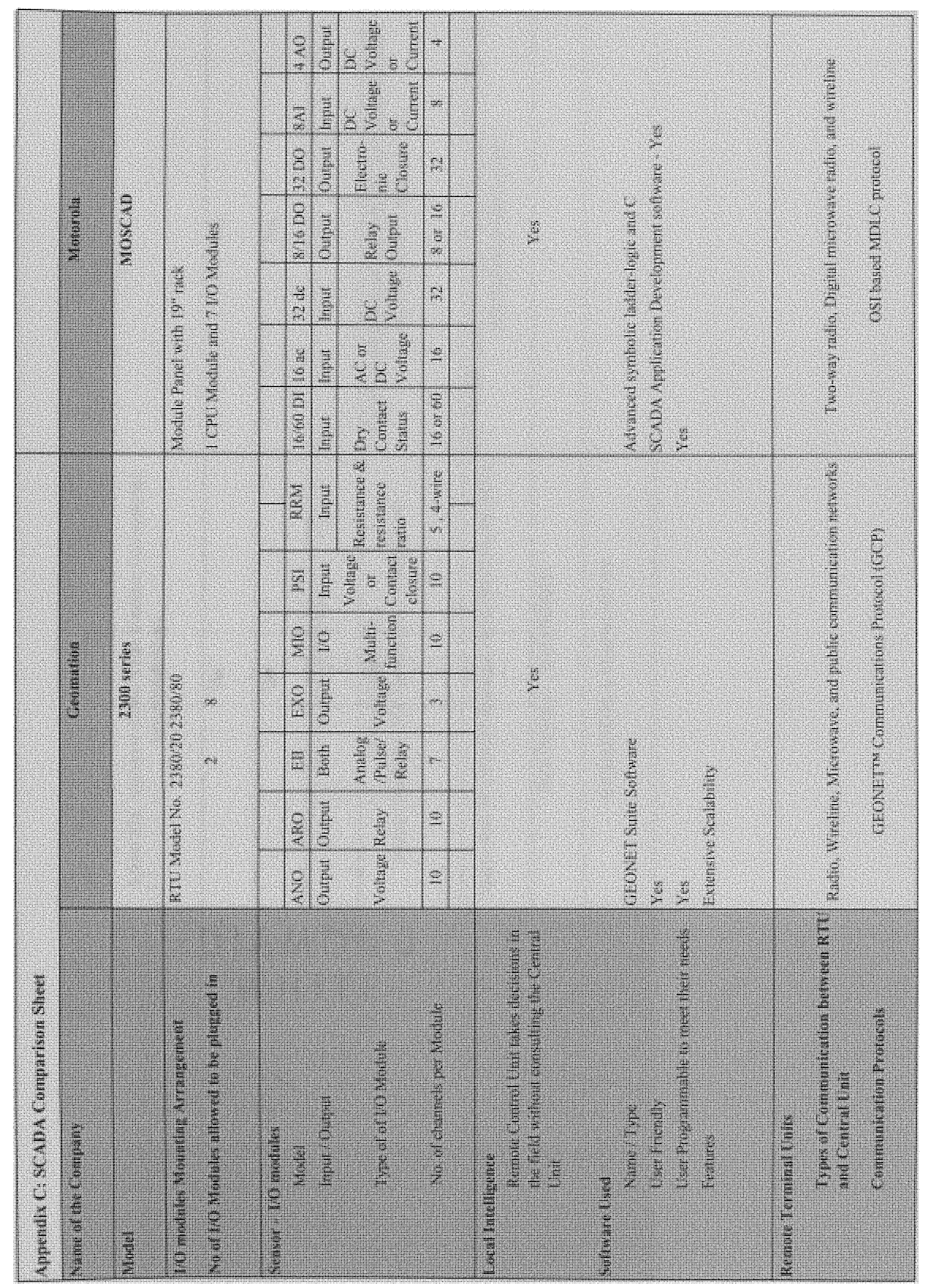

$\bar{g}$ 


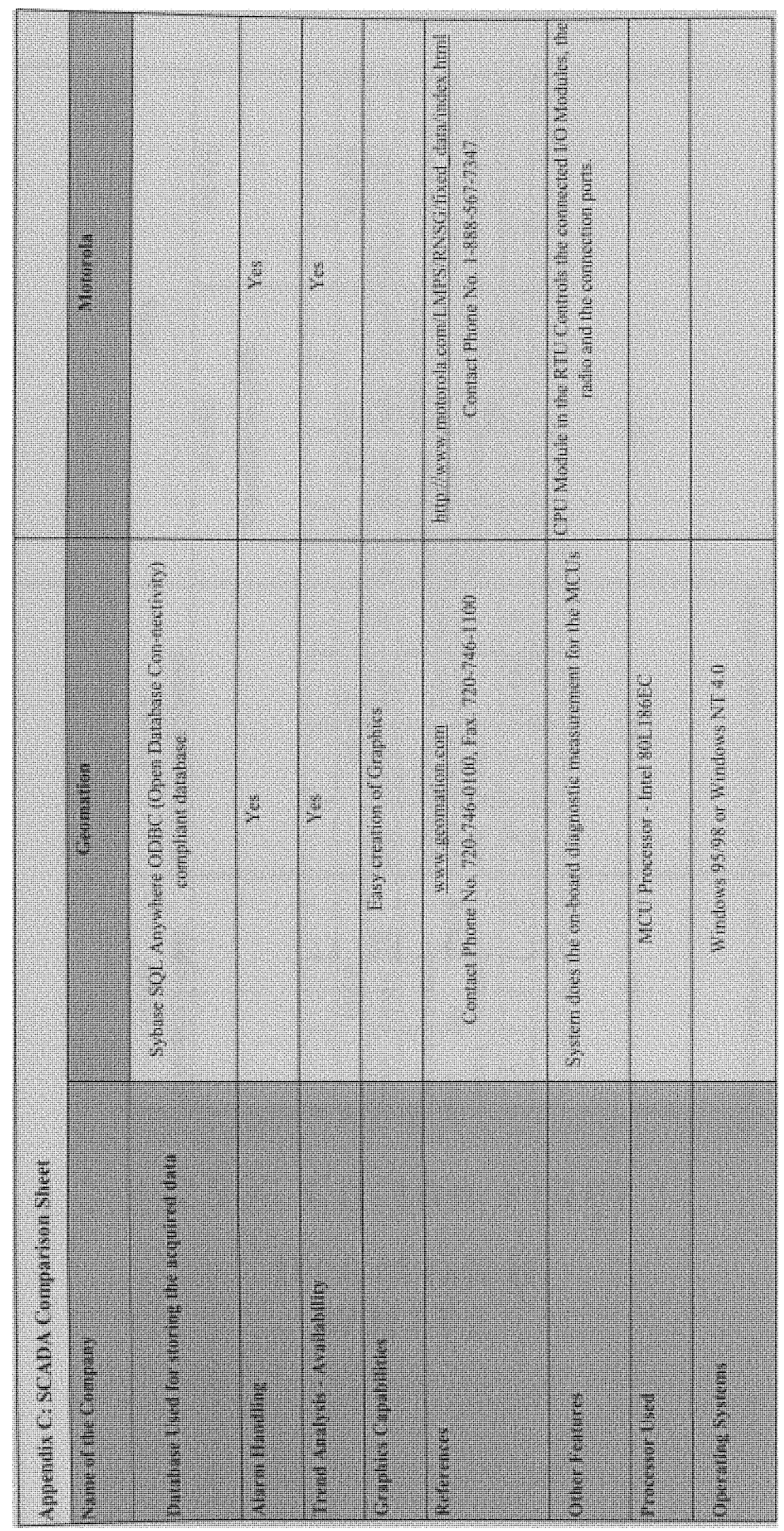




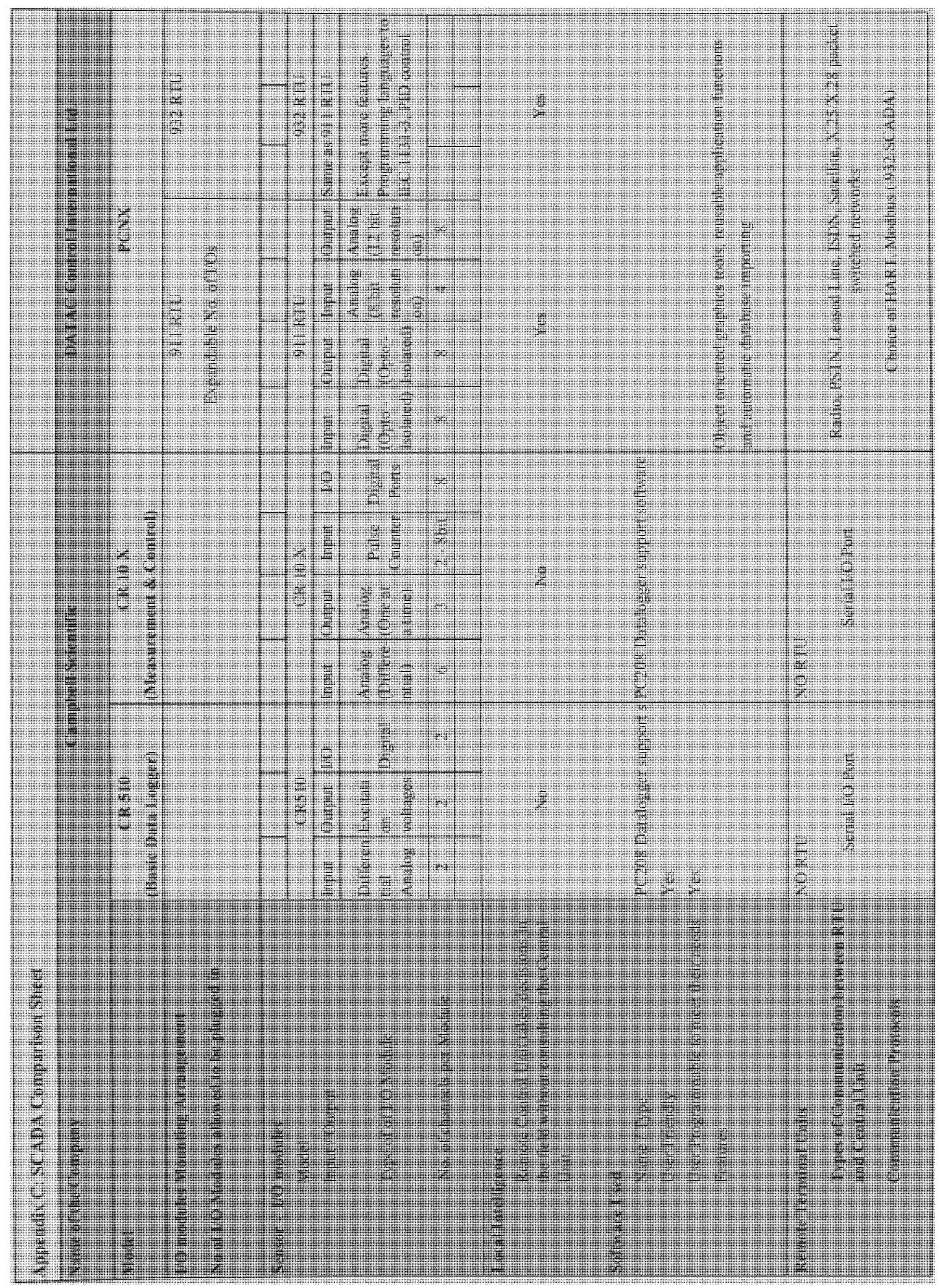




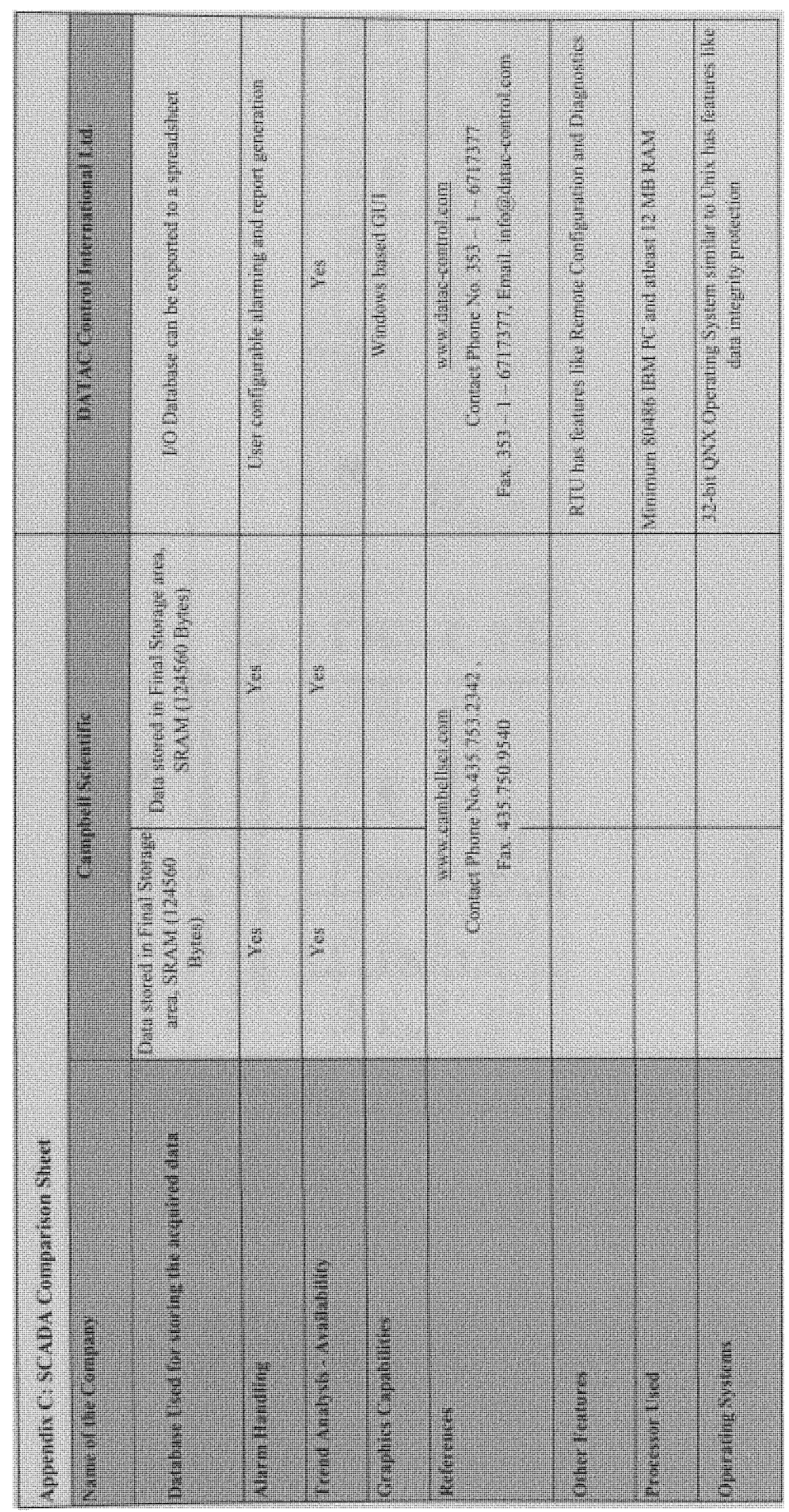




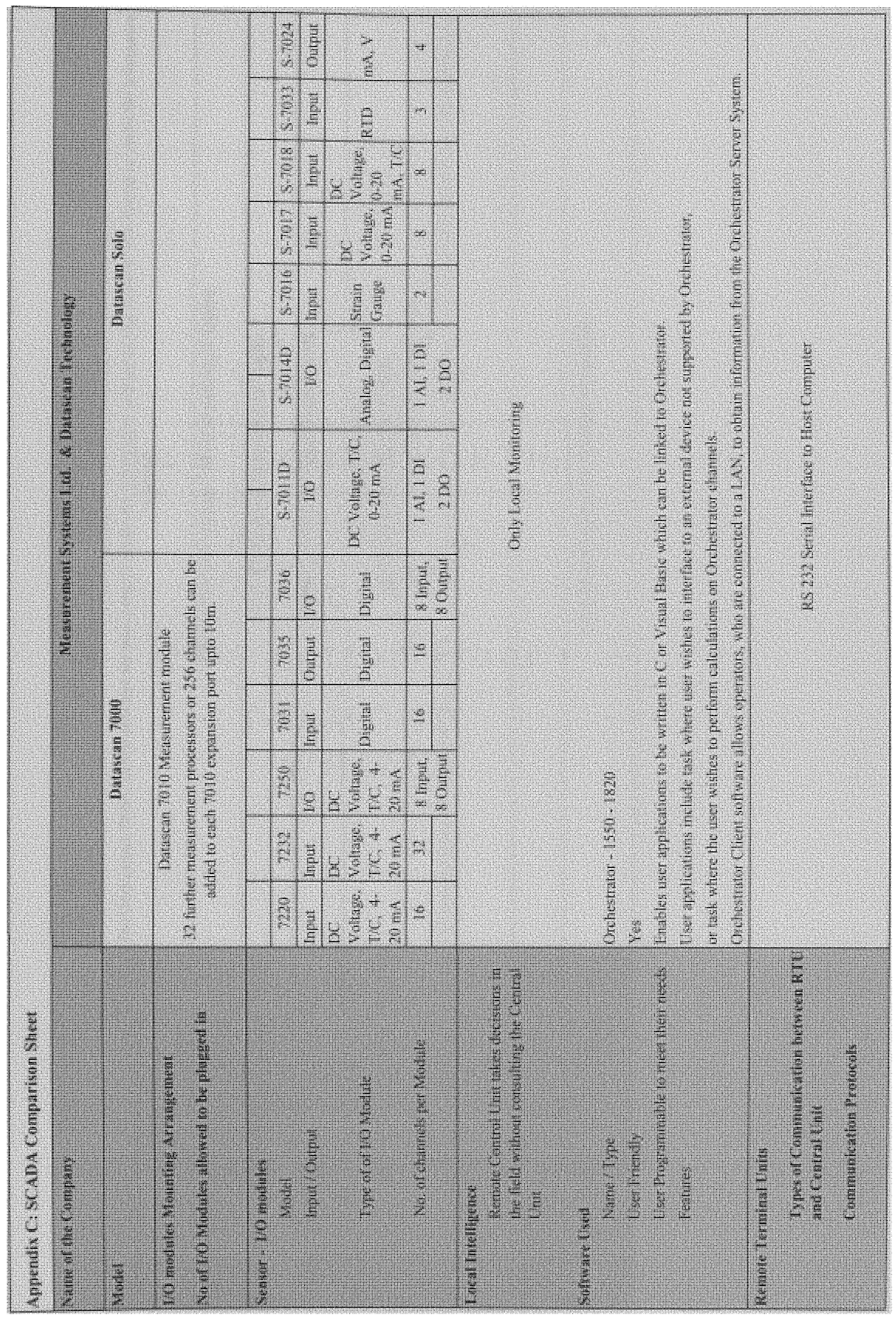




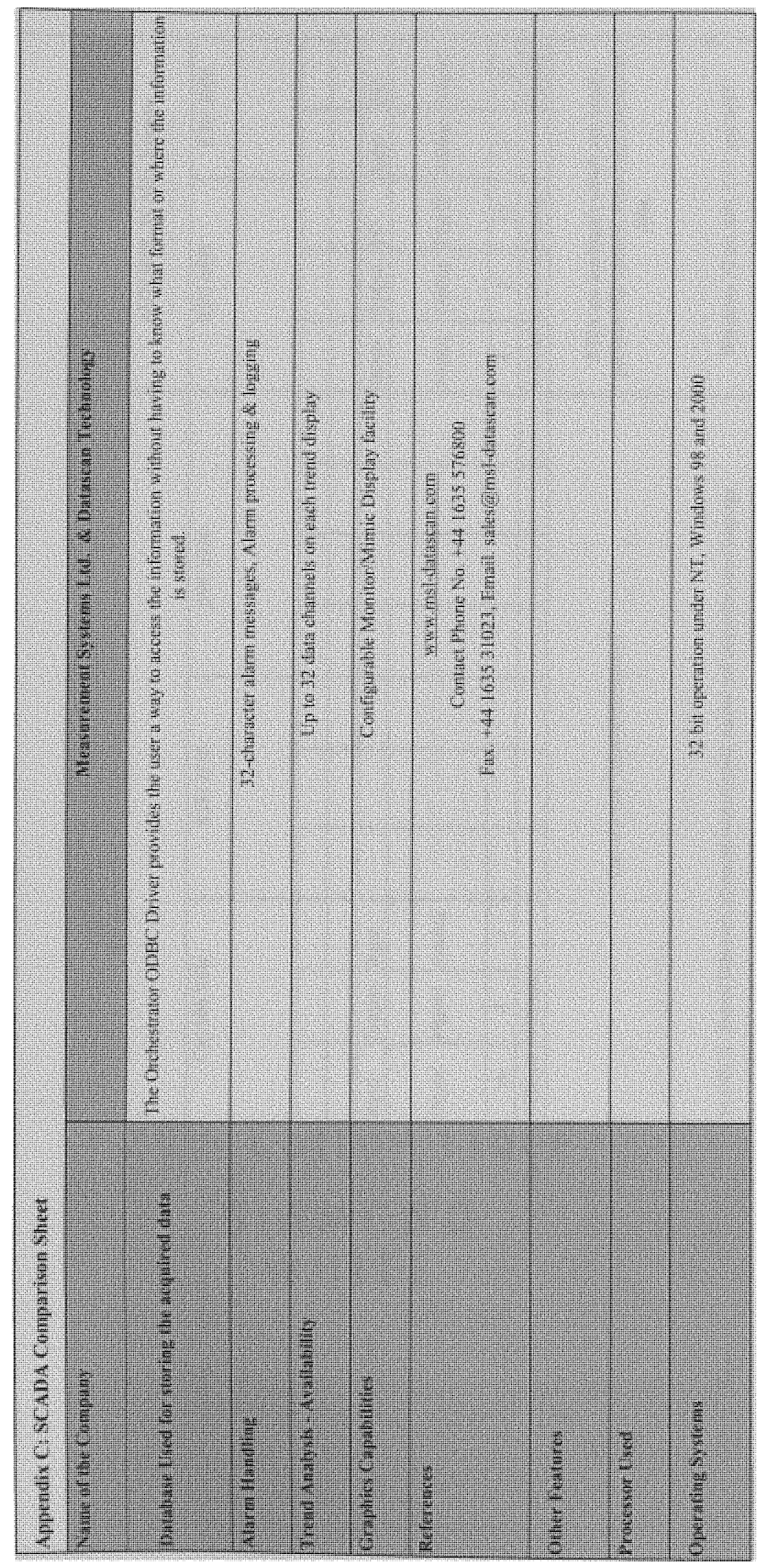




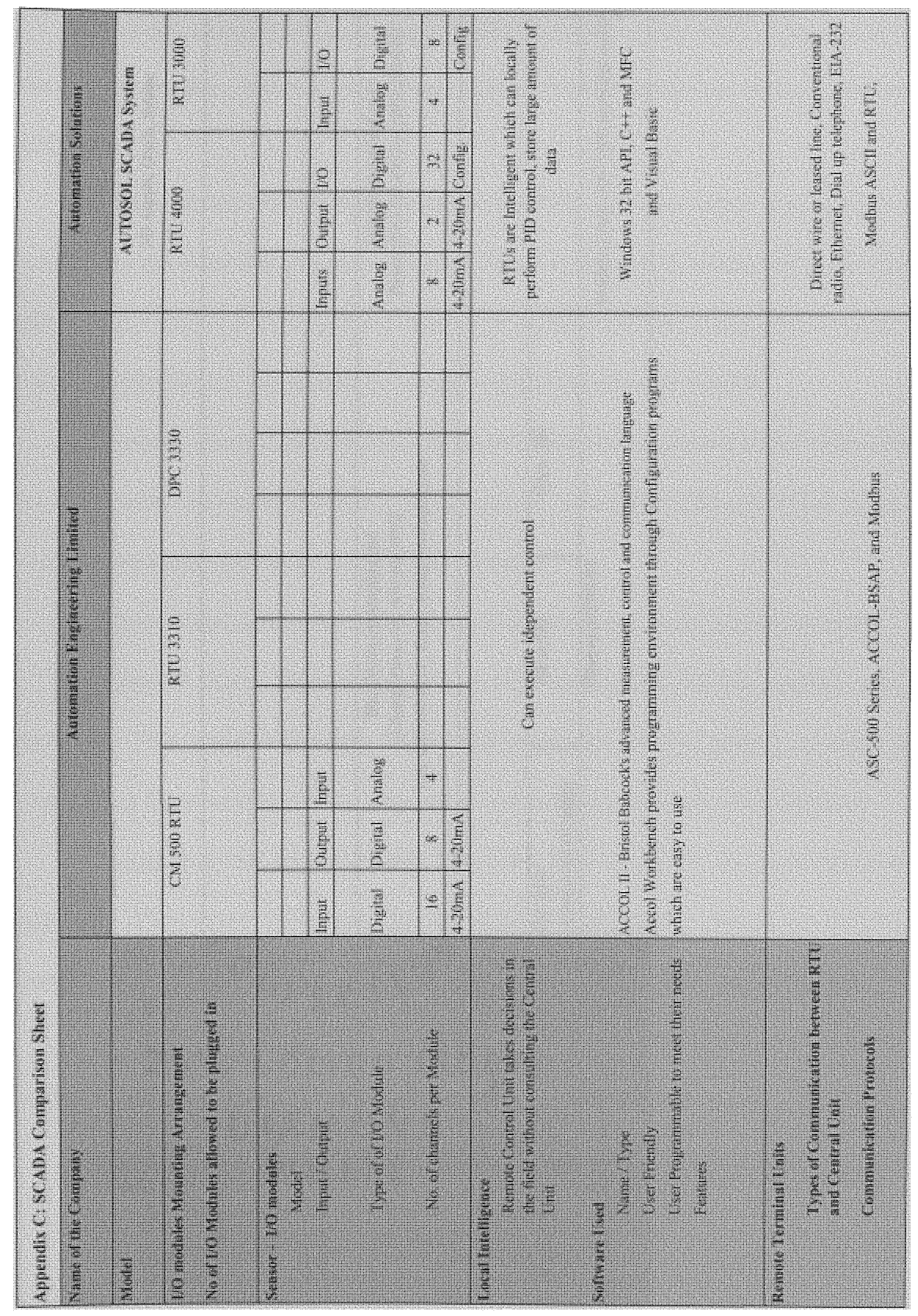




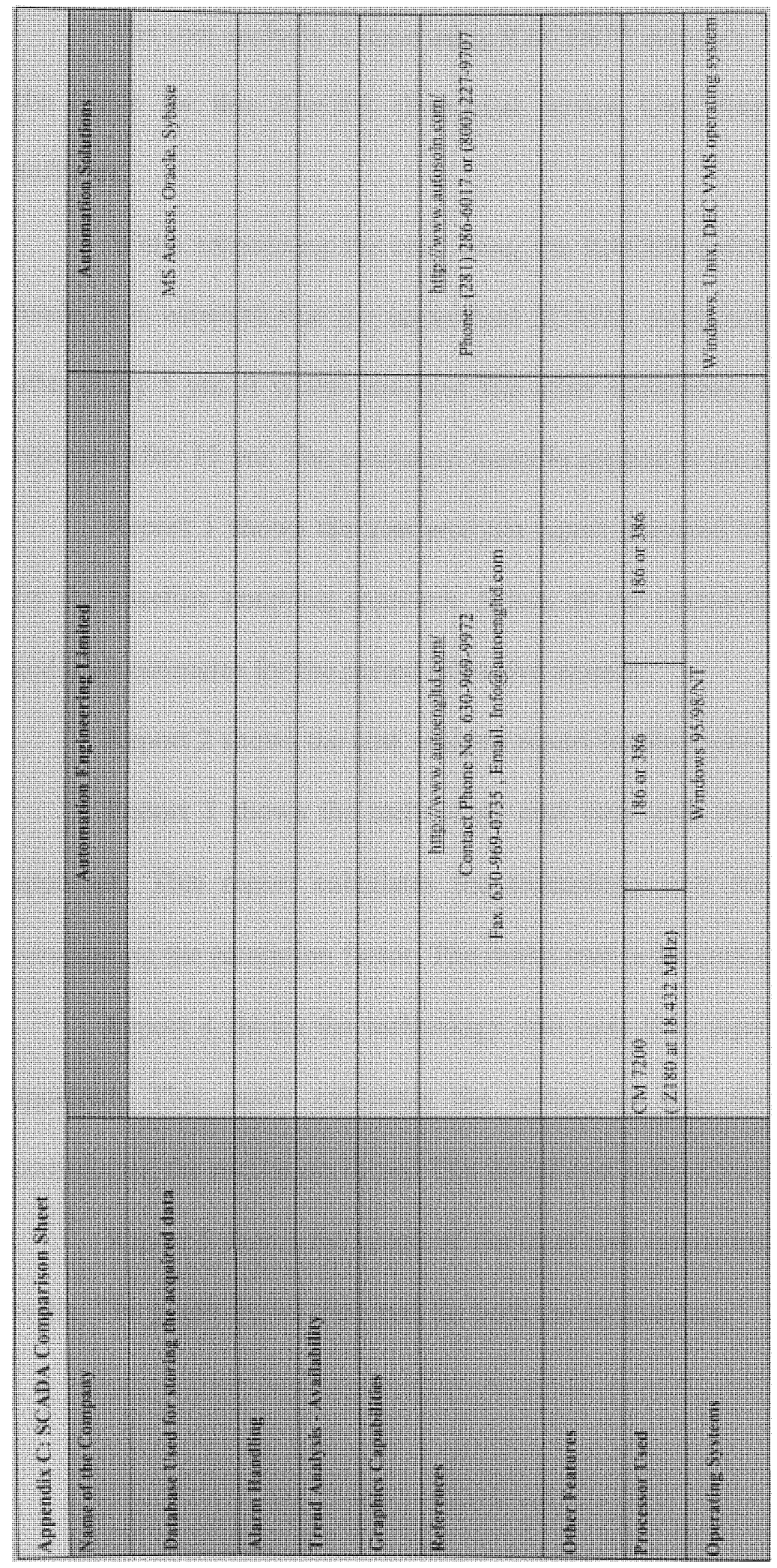




\section{Appendix D: Cost Analysis}

The cost analysis is done using RACER ${ }^{\mathrm{TM}}$ software. The Earth Tech, Inc holds all the rights for this RACER (Remedial Action Cost Engineering and Requirements) software.

While calculating the cost using the RACER software, some assumptions were made. It is assumed that the sensor and SCADA equipment would need to be replaced after every 5 yrs. It is also assumed that other equipments like pumps would need to be replaced after 15 yrs. The escalation factor is applied to the cost as well.

Report 1 shows the cost analysis report prepared by using the RACER software for the baseline sampling approach. It lists all the assemblies that contribute to the cost and their estimates for the period of twelve months.

Report 2 shows the cost analysis report for the multi-probe sensor approach.

Report 3 shows the technology cost over time report for the baseline sampling approach. This report calculates the technology cost for the period of thirty years considering the escalation factor. The initial cost is the cost calculated in Report 1.

Report 4 shows the technology cost over time report for the multi-probe sensor approach. This report calculates the technology cost for the period of thirty years considering the escalation factor. The initial cost is the cost calculated in Report 2.

Report 5 shows the graph of the total cost versus time of thirty years for hundred wells. The graph shows the trend for both the approaches. 


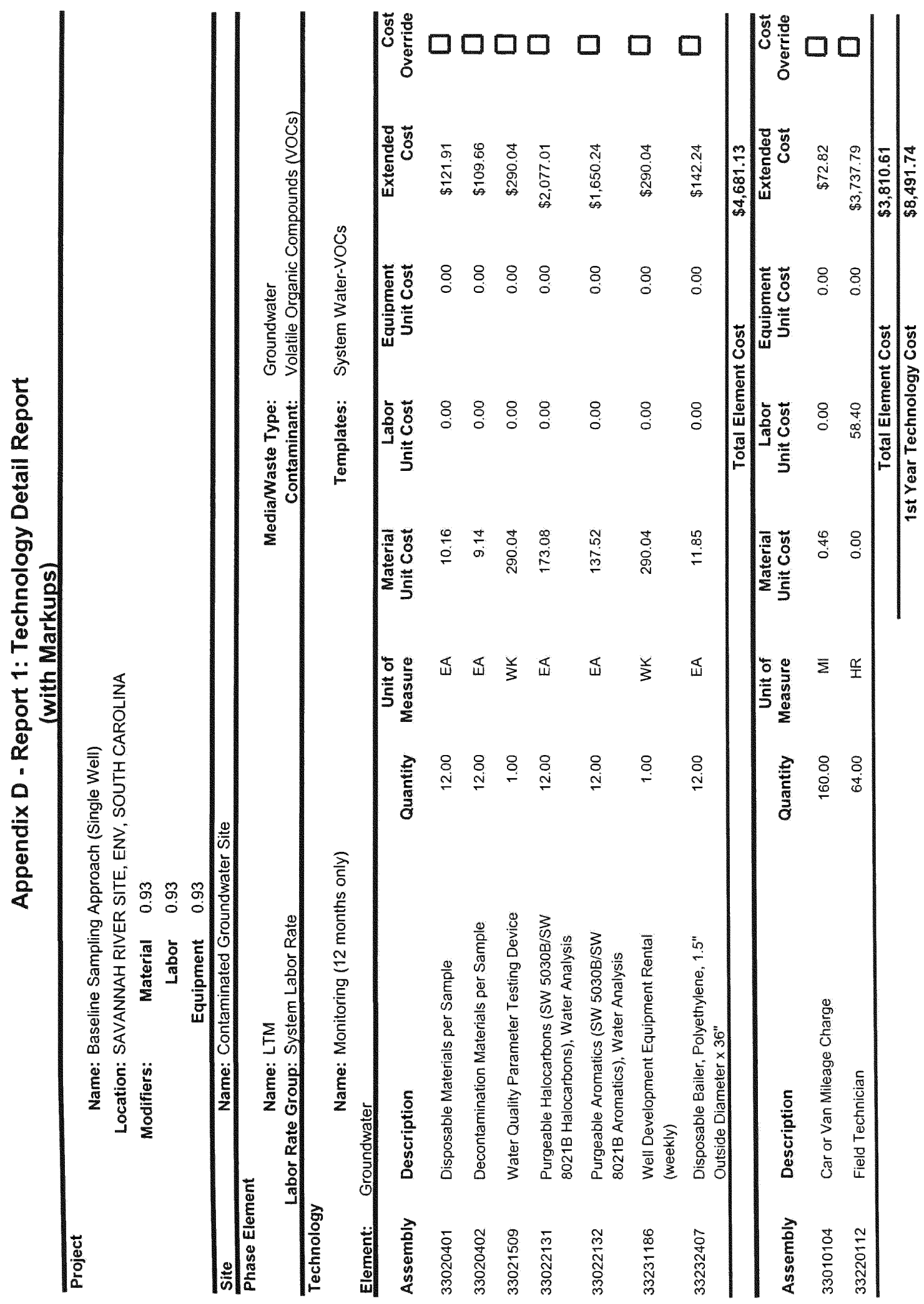




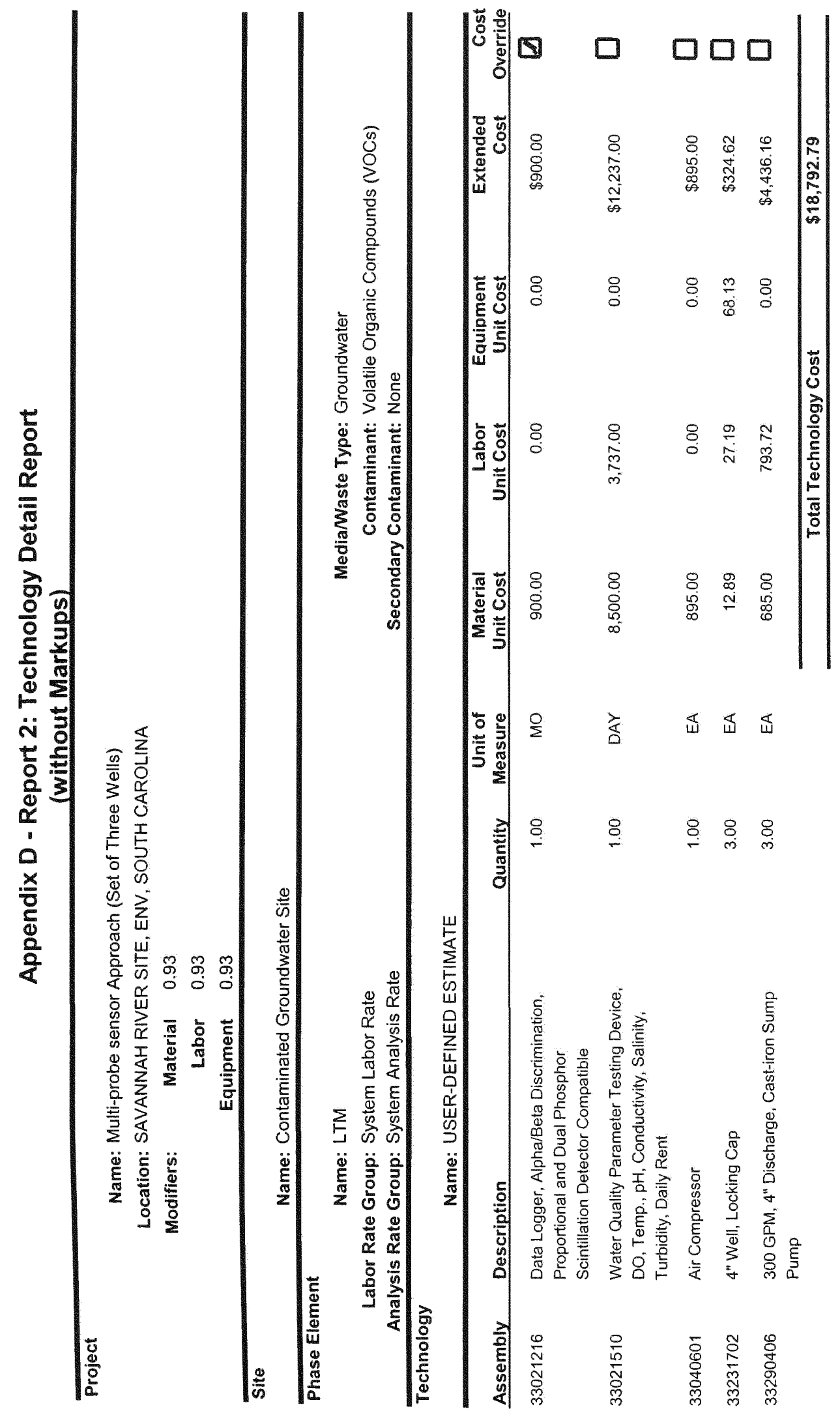




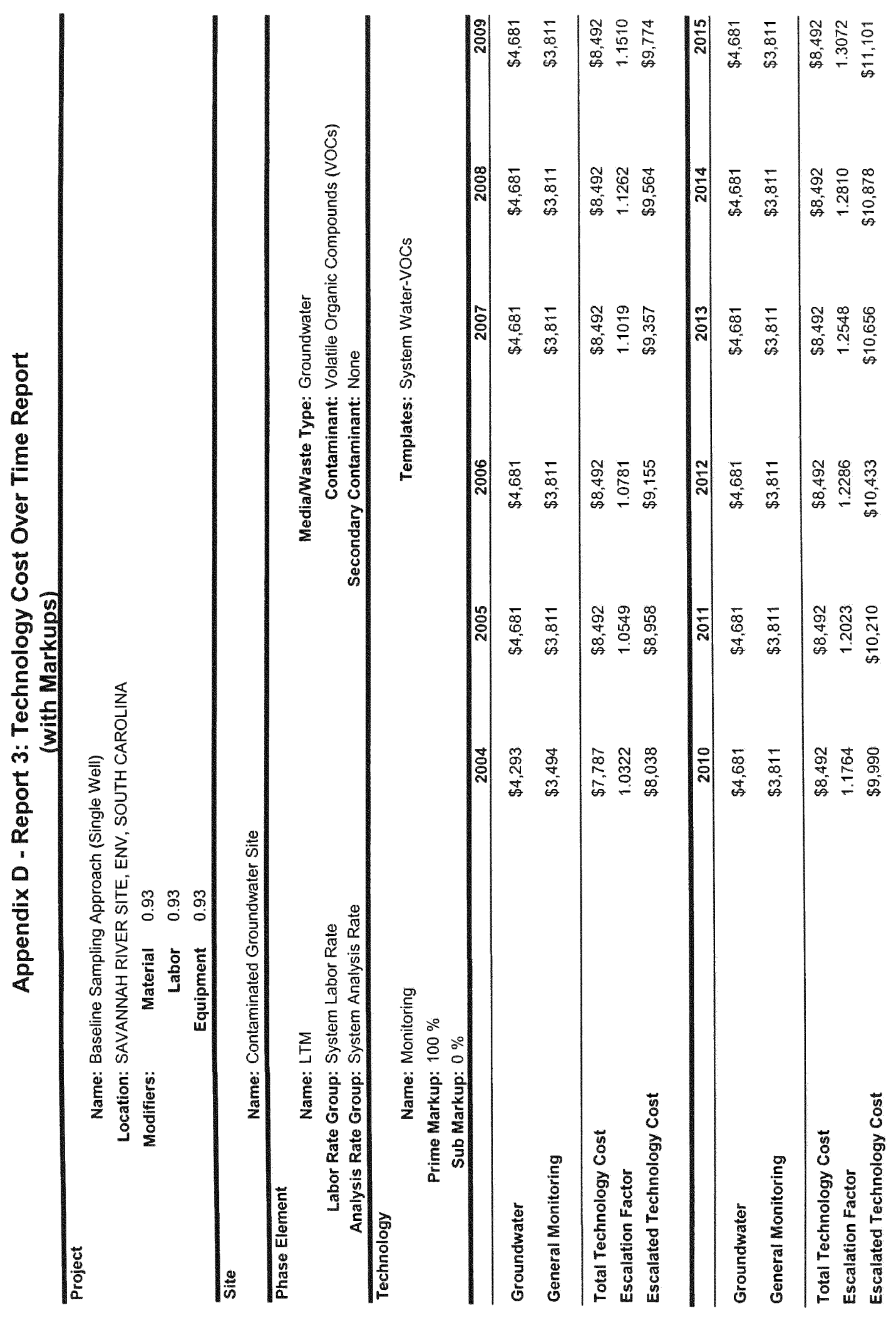




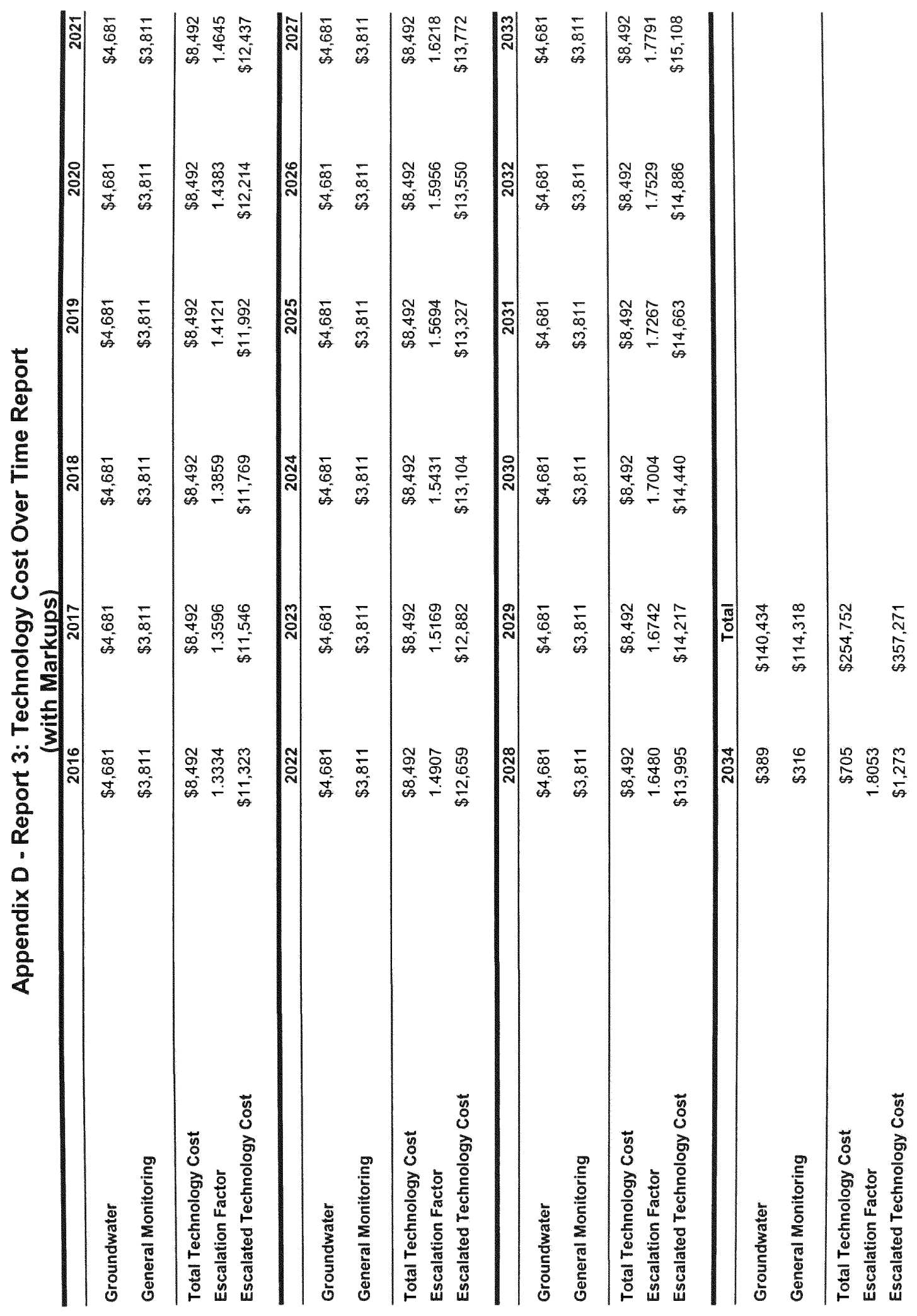




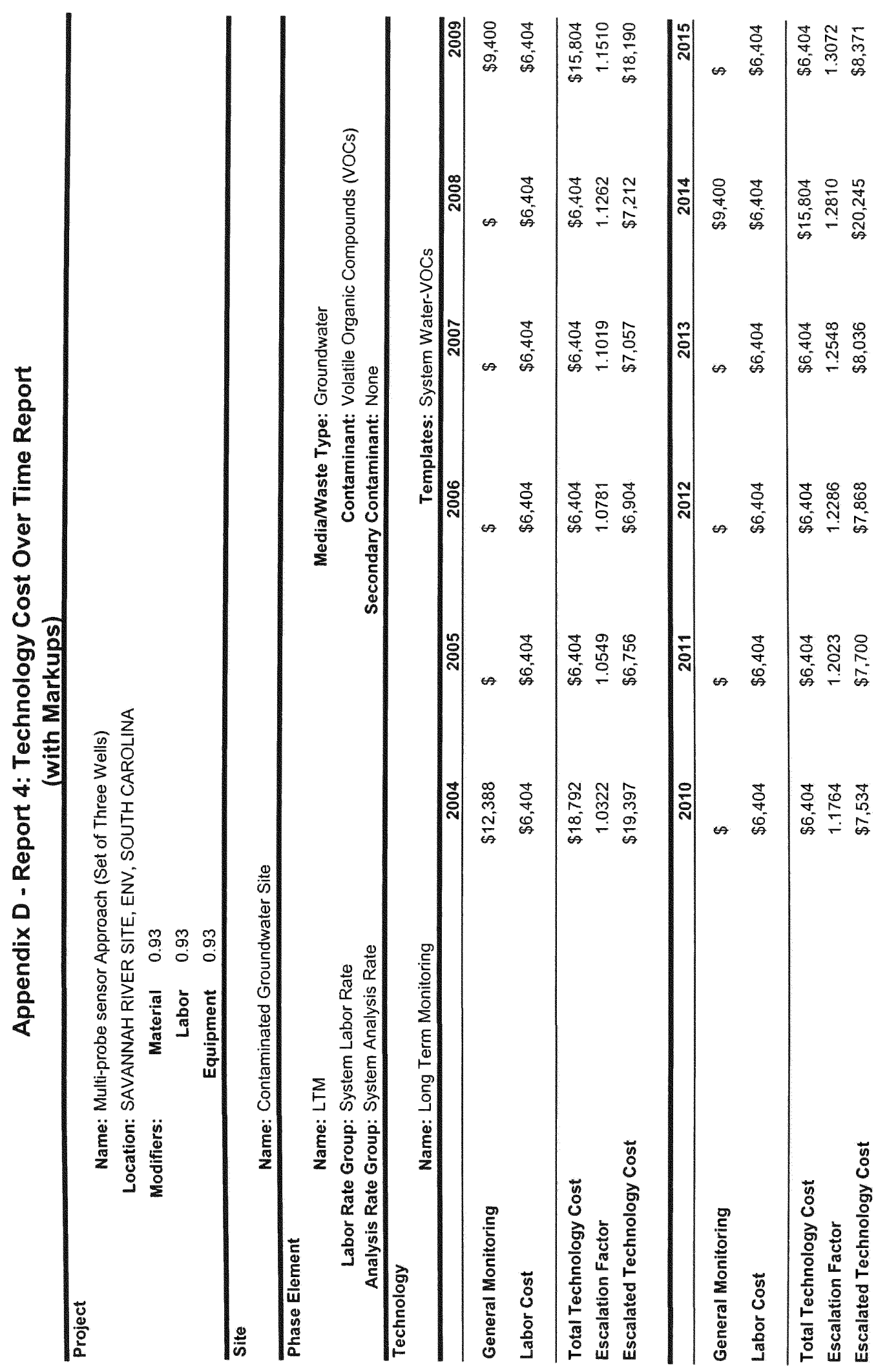




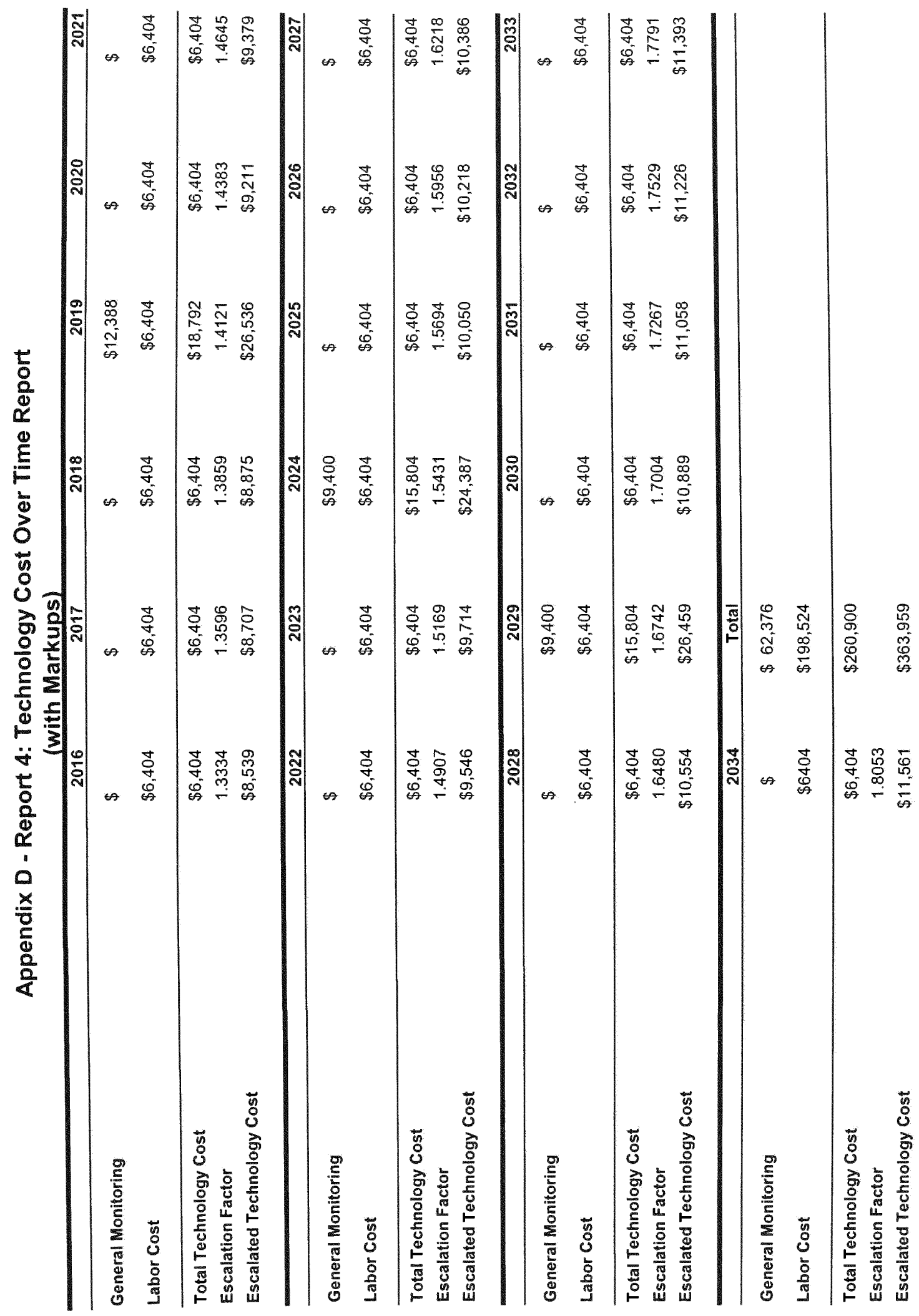




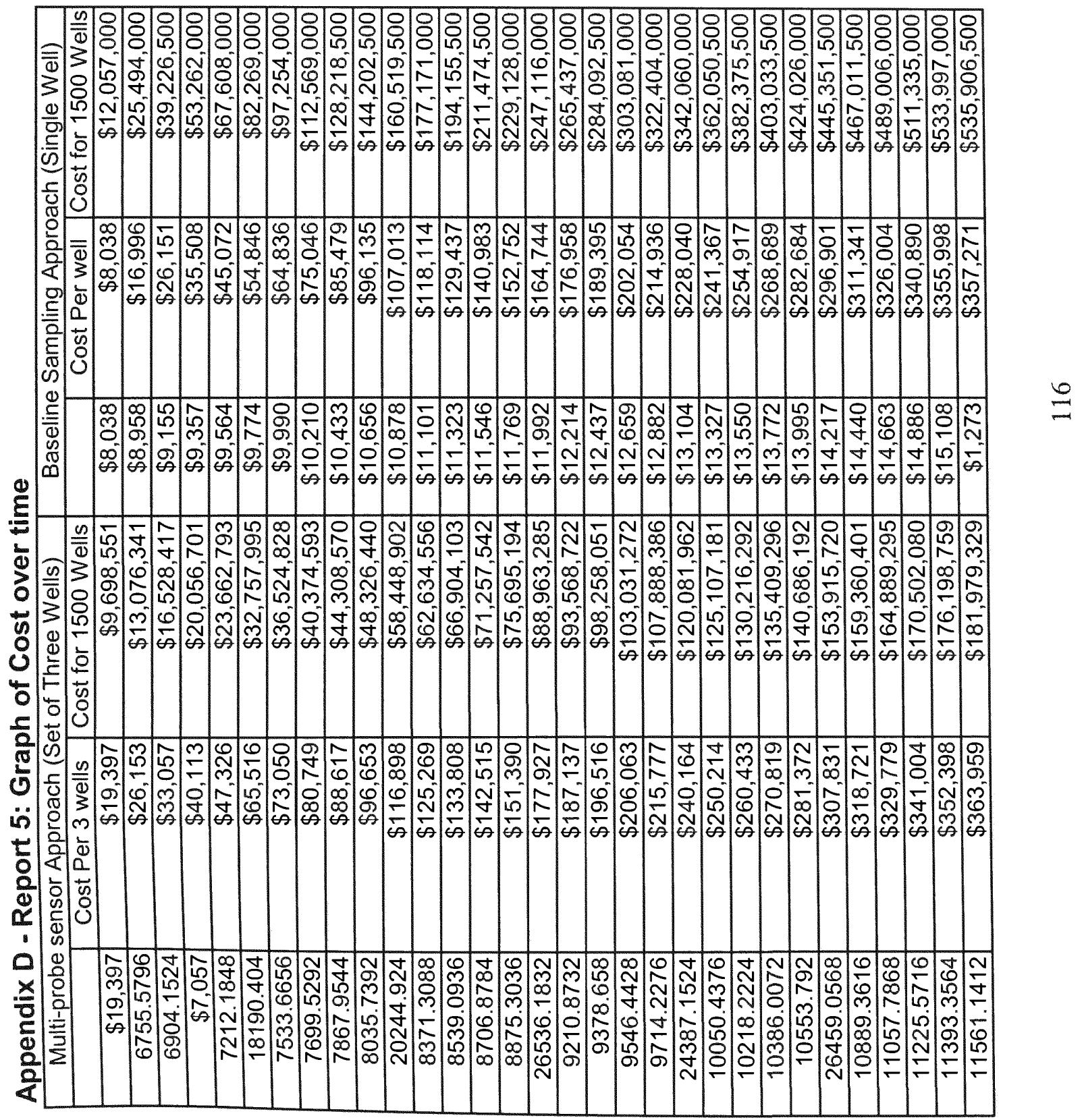




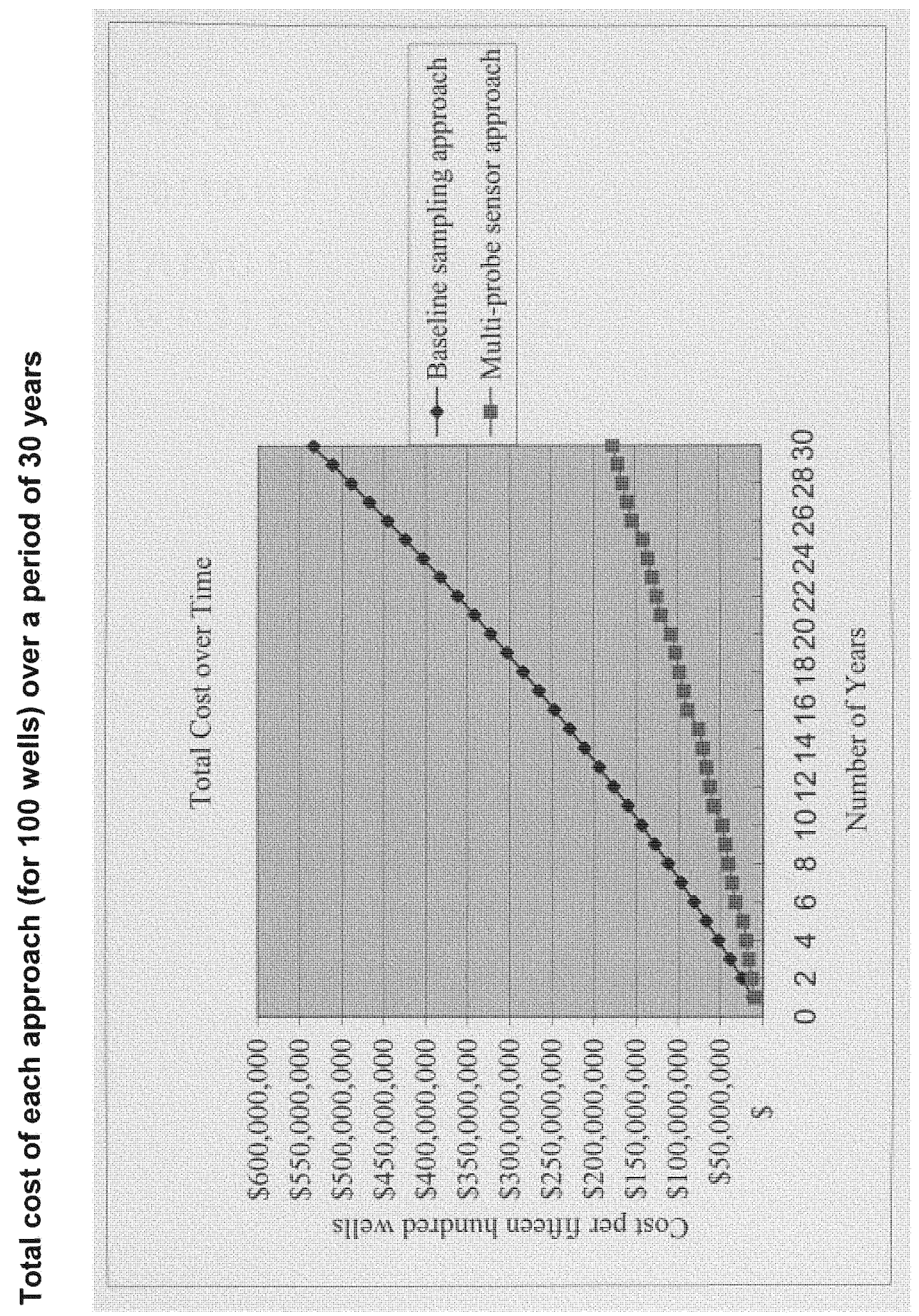

